\title{
Supramolecular networks of telechelic polymers
}

Małgorzata A. Bohdan 


\section{Thesis committee}

\section{Promotor}

Prof. Dr Jasper van der Gucht

Professor of Physical Chemistry and Soft Matter

Wageningen University

\section{Co-promotor}

Dr Joris H.B. Sprakel

Associate professor, Physical Chemistry and Soft Matter

Wageningen University

\section{Other members}

Prof. Dr John P.M. van Duynhoven, Wageningen University

Dr Tina Vermonden, Utrecht University

Dr Maarten M.J. Smulders, Wageningen University

Prof. Dr Sybrand van der Zwaag, Delft University of Technology

This research was conducted under the auspices of the Graduate School VLAG (Advanced studies in Food Technology, Agrobiotechnology, Nutrition and Health Sciences). 


\title{
Supramolecular networks of telechelic polymers
}

\author{
Małgorzata A. Bohdan
}

\section{Thesis}

submitted in fulfillment of the requirements

for the degree of doctor

at Wageningen University

by the authority of the Rector Magnificus

Prof. Dr A.P.J. Mol,

in the presence of the

Thesis Committee appointed by the Academic Board

to be defended in public

on Tuesday 6 September 2016

at 1:30 p.m. in the Aula. 
Małgorzata Anna Bohdan

Supramolecular networks of telechelic polymers

117 pages.

PhD thesis, Wageningen University, Wageningen, NL (2016)

With references, with summary in English

ISBN: 978-94-6257-867-8

DOI: $10.18174 / 386420$ 



\section{Contents}

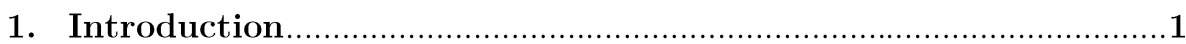

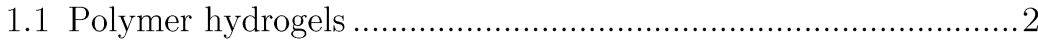

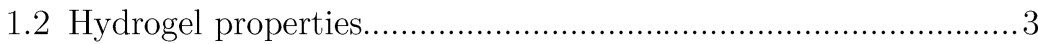

1.3 Supramolecular hydrogels.......................................................... 4

1.3.1 Hydrophobic assembly......................................... 5

1.3.2 Metal-ligand complexes ........................................ 6

1.4 Microscopic dynamics and rheology ……................................ 10



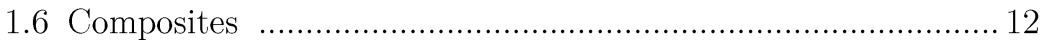

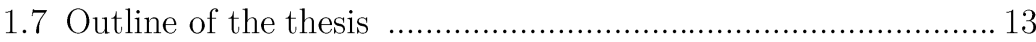

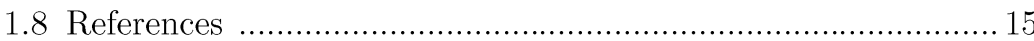

2. Multiple Relaxation Modes in Associative Polymer Networks

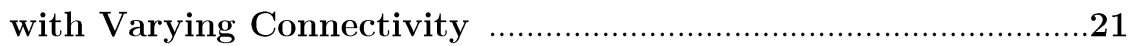

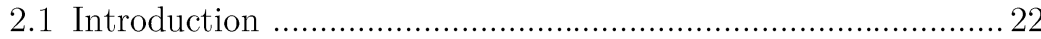



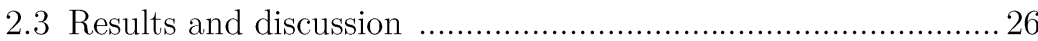

2.3.1 Oscillatory shear measurements ……….............22

2.3.2 Dynamic light scattering measurements .............30

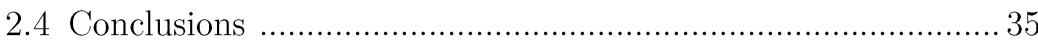

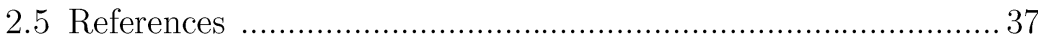

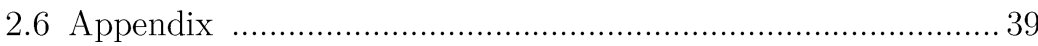

3. Critical Polymer Networks by Arrested Phase Separation ........41

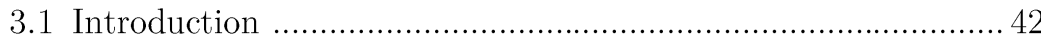


3.2 Materials and methods 43

3.3 Results and discussion ...................................................... 45

3.4 Conclusions .......................................................................... 54

3.5 References ….................................................................. 55

\section{Linear and Nonlinear Rheology of Metal-Coordinated} Supramolecular Networks ..................................................5 57

4.1 Introduction ..................................................................... 58

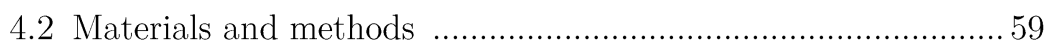

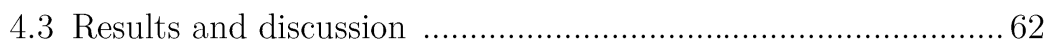

4.3.1 Terpyridine binding conditions ......................6 63

4.3.2 Metal ion ratio and concentration dependence ..63

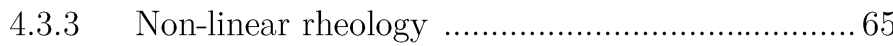

4.4 Conclusions ....................................................................... 70



5. Supramolecular Assembly of Self-Healing Nanocomposite Hydrogels ...................................................................... 73

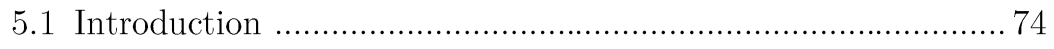

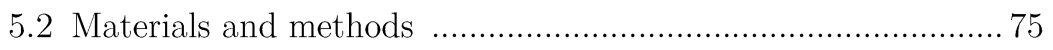

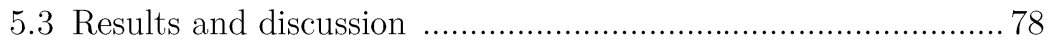

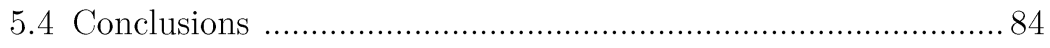

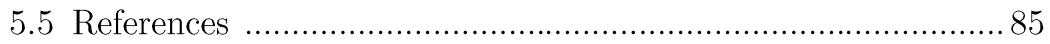

5.6 Appendix ..................................................................... 87

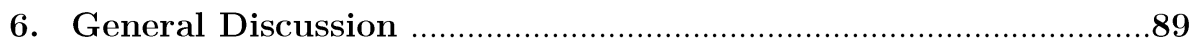

6.1 Phase separation \& mechanical properties .......................... 94

6.2 Critical gelation \& structure ......................................... 95 
6.3 Adhesives ..................................................................... 96

6.4 Telechelic-based double-network composites ....................... 97

6.5 Self-oscillating hydrogels ................................................... 100

6.6 References .................................................................. 102

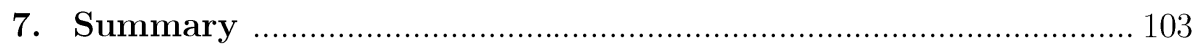

8. About the author ........................................................................ 107



10. Acknowledgements .............................................................. 111

11. Overview of completed training activities ............................. 117 
CHAPTER 1

Introduction 
Since the dawn of civilization, the ages have been defined by the materials that dominated society, from the stone and iron ages in the distant past to the silicon age of the moment. The start of the $\mathrm{XXI}^{\text {st }}$ century brought an enormous development in advanced materials research. Among others, materials scientists realized a breakthrough in ultrathin semiconductor production, such as graphene ${ }^{[1]}$, commercialized electronic paper ${ }^{[2]}$, designed ultralight carbon fibers ${ }^{[3]}$ and aerogels ${ }^{[4-7]}$. Material science is continuing its era of revolution resulting in more robust and practical solutions. However, even while expanding so rapidly on the application frontier, a true understanding of materials, in particular the relation between microscopic structure and macroscopic physical properties, remains incomplete. Such understanding is necessary to enable a sustainable growth of constant progress. Therefore, there is a need to look deep inside the nature of particular materials, applying to all branches of materials science. In this thesis, our aim is to take a closer look at the structure-property relation in polymeric hydrogels with reversible non-covalent crosslinks.

\subsection{Polymer hydrogels}

Hydrogels are aqueous materials derived typically from relatively low molecular mass compounds that when polymerized and cross-linked form a water-swollen network mechanically behaving as a solid. Such polymeric networks can be formed by many water-soluble monomers and also the crosslinking can occur by a variety of different mechanisms. Due to the variability in design, the mechanical properties of hydrogels can vary enormously, from soft and ductile to stiff and brittle. In some specific designs, these properties can be tuned by external stimuli, like temperature ${ }^{[8]}, \mathrm{pH}^{[9]}$ or photoradiation ${ }^{[10]}$. This rich performance is the reason why hydrogels have become so pervasive in recent years, finding application as gelling agents, thickeners and texture stabilizers for food, cosmetics or paints, as corrective contact lenses, or as scaffolds in biomedical applications such as bone ${ }^{[1-13]}$ or cartilage regeneration ${ }^{[13-19]}$. Even though their presence in households in the form of puddings is recorded already in century-old cookbooks, systematic studies into their physical nature started only several decades ago, pioneered by 
Nobel prize laureate Paul J. Flory ${ }^{[20]}$. Since then this effort has grown tremendously and succeeded in introducing many inspiring concepts, such as tough plastic composites, ${ }^{[9,14,15,18,21-25]}$ self-oscillating biomimietic gels, ${ }^{[26-28]}$ and a wide variety of soft-robots, ${ }^{[29,30]}$ to name just a few.

\subsection{Hydrogel properties}

The properties of hydrogels are determined by the interconnected network architecture. The required hydrogel properties can be tailored by selection of polymers of different stiffness or length, by varying the crosslink density or by the addition of additives such as nanoparticles. Third parameter that affects the network properties is the strength and dynamics of the crosslinks themselves. We consider bonds B that connect chains in the network by the association of two or more functional groups $\mathrm{F}$ :

$$
\ldots F+F \ldots \underset{k_{d}}{\stackrel{k_{a}}{\rightleftharpoons}} \ldots B \ldots
$$

where $k_{a}$ and $k_{d}$ stand for the rate constants of association and dissociation, respectively. The average lifetime of the bond is governed by its dissociation rate as:

$$
\tau_{B}=\frac{1}{k_{d}}
$$

A bond relaxation time $\tau_{B} \rightarrow \infty$, such as for covalent bonds, results in materials that, once cross-linked, cannot be processed anymore, but must rupture irreversible in order to yield. Such networks are called covalent or chemical rubbers or gels. Most non-covalent bonds, whose formation is based on physical or supramolecular interactions between specific moieties on the polymeric chain, will exhibit a finite dissociation rate which depends on the energy required to break the bond. Such materials can in principle dissociate and reform spontaneously, or in response to external triggers, such as temperature or $\mathrm{pH}$ adjustments ${ }^{[31,32]}$, or more sophisticated electrochemical treatments or light radiation ${ }^{[33]}$. This responsiveness gives rise to so called "smart", responsive and 
self-healing materials. Hydrogels built in this way are also called physical gels or supramolecular networks, and are the main topic of this thesis.

\subsection{Supramolecular hydrogels}

Supramolecular assembly occurs through non-covalent interactions such as H-bonding, van der Waal forces, donor-acceptor interactions, $\pi-\pi$ stacking, solvophobic forces, or metal coordination bonds ${ }^{[34]}$. Due to this diversity of interactions the bond lifetimes can vary enormously, from a few milliseconds to hours or even days ${ }^{[35-38]}$ depending on the association energy of the bond of interest, which typically follow Arrhenius kinetics. In order to form a functional network it is necessary that the building molecules assemble in a specific and directional way rather than into random aggregates. Nature makes ample use of reversible supramolecular interactions; for example, H-bonding between specific nucleotides drive the double helical structure of hybridized DNA and the nonpermanent character of this bonding is crucial for further replication.
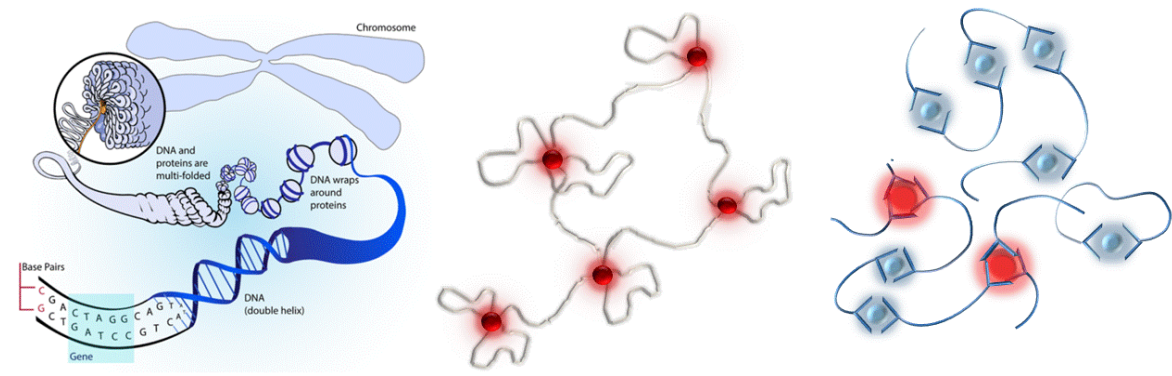

Figure 1.1: Examples of supramolecular assembly of various polymers. (a) DNA nucleotides H-bonding assembly in double helix (after www.ck12.org), (b) hydrophobically driven assembly of telechelic polymers, (c) metal-ligand complexation based assembly of telechelic polymers 
Likewise, in our cells, supramolecular polymerization of proteins results in long supramolecular filaments, e.g. from actin or tubulin, which are essential for cell rigidity, trafficking and polarization. These biological examples are a great inspiration for synthetic design and, indeed, since the recognition of the role of supramolecular chemistry by the 1987 Nobel Committee, a great number of new types of reversible assemblies were discovered and developed, often finding a basis in nature ${ }^{[39,40]}$. A special class of supramolecular hydrogels is formed by linear polymers di-functionalized with associating groups at both ends alone. These so-called telechelic polymers can spontaneously form supramolecular networks by association of the end groups into nodes, while the soluble middle blocks form the connecting chains. Various telechelic polymers have been reported, with different kinds of end groups, resulting in essentially similar networks whose node connectivity, spacing and dissociation rate vary ${ }^{[31}$, $38,41-46]$. Below we discuss two kinds of association motifs used in this thesis in more detail.

\subsubsection{Hydrophobic assembly}

Perhaps the most studied designs are based on hydrophobic interactions. With the appropriate hydrophilic-lyophilic balance, these polymers form homogeneous networks of so-called flowerlike micelles (Fig.1b). The association strength of these polymers can be tuned by varying the length of the solvophobic groups and the water-soluble spacers.

The simplest hydrophobically modified telechelics are designed as a triblock (ABA) copolymer, with a soluble middle block (B) and hydrophobic associating end blocks (A). As the backbone molecule, any water-soluble linear chain can be used, whereas the hydrophobic part is usually built from small hydrocarbon or fluorocarbon entities ${ }^{[41,42,47-50]}$.

When dissolved in water, hydrophobically modified telechelics will minimize the unfavorable contacts of solvophobic moieties with the solvent by exhibiting microphase separation. By contrast, the water-soluble spacer prefers to remain hydrated. The balance between these opposite forces leads to the spontaneous formation of well-defined structures called flower-like micelles, which feature a 
distinct hydrophobic core and a water swollen star-like polymeric corona. The properties of the micelles depend on the precise structure of the triblock polymer; for example, the number of sticky ends per micellar core - the aggregation number - increases as the end block size is increased or the hydrophilic spacer is reduced in length ${ }^{[51]}$. Also, longer hydrophobic stickers result in an increase in the energy barrier for dissociation, which involves hydrating a hydrophobic block, and thus increases the average residence time of the sticky end within the micelle core ${ }^{[42,52,53]}$.

Depending on the concentration of the polymer we can distinguish four stages of structure development. At extremely low concentrations telechelics appear as a solution of unimers, while above the critical micelle concentration (CMC), the hydrophobic end-blocks will cluster together, leading to the formation of flowerlike micelles. The possibility of the middle blocks to bridge between different micelles gives rise to an attractive energy of entropic origin between the micelles. This can lead to clustering of micelles, and even to phase separation into two macroscopic phases, where one phase consists of concentrated, densely packed interconnected micelles and the second phase of excess solvent with few unimers and micelles. By further increasing the concentration beyond the binodal, we reach a phase of homogeneous, sample-spanning and highly viscoelastic transient networks with associative connections between the micellar cores. The phase behavior of such systems was widely discussed by many groups, where temperature and concentration dependence, functionality degree or surfactant addition was studied [41, 42, 47, 52-55]. Hydrophobically modified telechelics are in broad use as, for example, commercial thickeners in paints, cosmetics or oildrilling fluids. From an academic point of view, their particular interest is as a model system for studying in detail the formation, structure, dynamics and mechanics of self-assembled reversible polymer networks.

\subsubsection{Metal-ligand complexes}

In addition to hydrophobically-driven association, in this thesis we also use telechelics with coordination bonds as the associating motif. This approach starts with polymers whose chain ends have been functionalized with a ligand 
group, which form relatively strong, highly directional and stable metal-ligand complexes in the presence of metal ions (Fig. 1.1.c). The lifetime and strength of such complexes $\tau_{B}$ depend on the choice of the metal-ligand pair. This is illustrated, for example, by the process of oxygen transportation in living organisms. Iron ions in the heme group of hemoglobin bind to oxygen which is the primary function in living systems; yet, binding occurs more strongly to carbon monoxide or cyanide anions, with which it forms much more stable, almost irreversible complexes, which thus results in the inhibition of oxygen uptake and can cause severe pathologies.

A wide range of chemical ligands are available, of which the binding strength can be tuned, for example by choosing the proper metal ion or by changing $\mathrm{pH}$ or ionic strength in aqueous solutions. Complexation of the specific ligand $L$ to the metal ion $M^{n+}$ is defined by:

$$
M^{n+}+n L \underset{k_{d}}{\stackrel{k_{a}}{\rightleftharpoons}} M L_{n}, \quad K=\frac{\left[M L_{n}\right]}{[M]\left[L_{n}\right]}
$$

where $K$ is the equilibrium constant known commonly as the stability constant. In this thesis we consider linear chains functionalized with ligand groups at both ends, i.e. coordination telechelics; other geometries have been described in literature, e.g. using multivalent building blocks based on multiarm star polymers decorated with ligand groups ${ }^{[56-59]}$.

In Chapter 3 and Chapter 4 we present a study on terpyridine-based telechelic polymers that form tridentate coordination complexes with bivalent transition metal ions $\left(\mathrm{M}^{2+}\right)$ and/or trivalent lanthanides $\left(\mathrm{M}^{3+}\right)$. For metal ions that form complexes with $n=2$, only chain extension takes place. This can increase the solution viscosity by increasing the number of entanglements (Fig1.2.a), but does not lead to the formation of networks with coordination-bonds as nodes. Theoretically, to achieve this, we need coordination bonds with $n>2$. This implies using a mixture of bi- and trivalent metal ions, or the trivalent exclusively. At the right stoichiometry, bivalent metal ions again act as chain extenders, whereas trivalent ions play the role of the crosslinks. By tailoring the stoichiometry between $2<n<3$ (Fig. 1.2.b) we tune the density of the nodes 
from poorly crosslinked at $n \rightarrow 2$, to highly crosslinked at $n \rightarrow 3$. By using only trivalent metal ions, $n=3$, we form a network where each coordination group is part of a crosslinking node (Fig. 1.2.c). Formation of a stable network, however, relies also on other, more specific characteristics than only the valence of the metal ions. As we show in this research, additional mechanisms of association, e.g. hydrophobic or $\pi-\pi$ stacking interactions between metal-ligand pairs takes place.

The nature of the polymer network formed by coordination polymers depends crucially on the lifetime of a metal-ligand bond, which is related to the dissociation rate, $\tau_{B}=1 / k_{d n}=K_{n} / k_{a n}$. For strongly binding metals, which exhibit a low rate of dissociation as compared to the bond formation rate, the coordination bond is effectively irreversible. By contrast, a high rate constant implies very dynamic assembly, where the metal ions continuously associate and dissociate with the ligand groups under the action of thermal fluctuations.
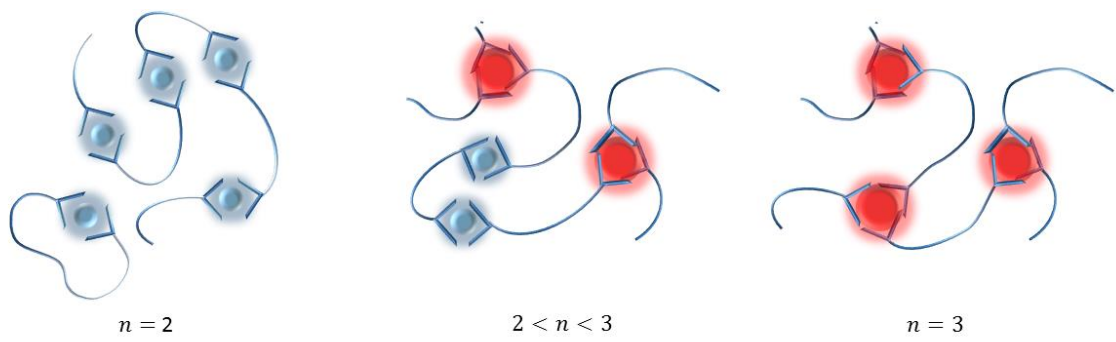

Figure 1.2: Metal-ligand complexation of terpyridine functionalized telechelic polymer: (a) complexation with divalent $\left(M^{2+}\right)$ transition metal ions, (b) complexation with mixture of divalent $\left(\mathrm{M}^{2+}\right)$ transitions metal ion and trivalent lanthanides $\left(\mathrm{M}^{3+}\right),(\mathrm{c})$ complexation with trivalent lanthanides $\left(\mathrm{M}^{3+}\right)$.

Formation of coordination complexes occurs in two stages. First, an outer sphere complex is formed, in which the hydrated metal ion associates with the ligand. Subsequently, the hydration water is removed from the metal ion; this dehydration is usually the rate-determining step, and differs strongly from one metal ion to another (see Table 1.1). 


\begin{tabular}{|c|c||c|c|}
\hline Metal ion & $k_{-w}\left(s^{-1}\right)$ & Metal ion & $k_{-w}\left(s^{-1}\right)$ \\
\hline$P b^{2+}$ & $7 \cdot 10^{9}$ & $F e^{2+}$ & $4 \cdot 10^{6}$ \\
\hline$C u^{2+}$ & $1 \cdot 10^{9}$ & $F e^{3+}$ & $2 \cdot 10^{2}$ \\
\hline$C d^{2+}$ & $3 \cdot 10^{8}$ & $\mathrm{Ni}^{2+}$ & $3 \cdot 10^{4}$ \\
\hline $\mathrm{Zn}^{2+}$ & $7 \cdot 10^{7}$ & $\mathrm{Co}^{2+}$ & $2 \cdot 10^{6}$ \\
\hline
\end{tabular}

Table 1.1: Dehydration rate constants for a selected transition metal ions ${ }^{[60]}$

The rate constant of complex formation can be described as $k_{a i}=k_{-w} \mathrm{~K}_{o s}$ where $k_{-w}$ is the dehydration rate constant characteristic for a particular metal ion and $\mathrm{K}_{o s}$ the outer sphere complex equilibrium constant, which depends mainly on the charges of the ligand and the metal ion and on the ionic strength. It follows that we can estimate the coordination bond lifetime as $\tau_{B}=$ $1 / k_{d n} \approx K_{n} / k_{-w} \mathrm{~K}_{o s}$. Table 1.1 collects dehydration rates for a number of metal ions; from these numbers it is clear that the lifetime of the bonds can vary by many orders-of-magnitude from one metal ion to another. This fact, and the sensitivity of the bond strength to other factors, like solvent quality or temperature, make coordination chemistry a powerful tool in supramolecular chemistry. Effectively used, it allows the formation of highly tunable, selfhealing polymeric networks with bond lifetimes that can be varied in a very wide range, from very dynamic to virtually permanent.

Metal-coordination supramolecular materials have been broadly studied in recent years for their application as sensors ${ }^{[61-63]}$, self-healing coatings ${ }^{[64,65]}$ or catalysts ${ }^{[66,67]}$. Commonly used ligand groups include pyridine derivatives, such as bipyridine or terpyridine ${ }^{[68,69]}$, and dipicolinic acid ${ }^{[70-72]}$. Various hydrogels based on metal coordination bonds have been studied, with different polymer architectures, such as stars ${ }^{[34,62,73,74]}$ or side-grafted chains ${ }^{[75]}$. Interestingly, it was found that hydrogels of ligand-functionalized star polymers crosslinked by metal ions always show similar mechanical properties, regardless of the number of arms. Recent findings suggest that terpyridine modified polymers cross-link not only due to coordination bonds, but also the tendency to associate by 
additional forces, such as hydrophobic interactions, leading to higher-order associated structures ${ }^{[74]}$. This will be discussed in Chapter 3 and 4 of this thesis.

\subsection{Microscopic dynamics and rheology}

The microscopic dynamics and global mechanical properties of polymeric networks formed by telechelic polymers are influenced strongly by several parameters, such as the bond lifetime, the number concentration of chains and the spatial connectivity of the network ${ }^{[53,76]}$. When the concentration is low, association of the end blocks leads to the formation of small polymer clusters or flowerlike micelles. Just above the overlap concentration, these clusters become interconnected by the formation of polymer bridges, driven by the entropy of end-exchange. At some concentration, which we denote as the percolation point, this leads to a sample spanning network with a finite shear modulus and a viscosity that increases strongly with increasing concentration ${ }^{[42,47,53]}$.

Often, these properties of telechelic networks are probed using rheological experiments. Here we consider two important parameters that can be extracted from such measurements, and which are important to describe the linear dynamics and mechanics. The mechanical strength of this type of assembled network can be described by the value of the shear elastic modulus $G^{\prime}$ that, by following the classical rubber theory, depends linearly on the number of elastically-active chains between network nodes per unit volume $N$ as $G^{\prime} \propto$ $N k_{B} T$. Also viscoelastic relaxation can be measured, that is directly correlated with the association strength of the functional end-groups. For example, for PEO chains modified with two alkyl tails, the viscoelastic relaxation time increases exponentially with the length of the tails, which can be attributed to an Arrhenius-like activated process with an energy barrier which increases linearly with alkyl length ${ }^{[42,53]}$. An alternative method to probe the dynamic properties of these transient polymer networks is to use dynamic light scattering (DLS). Whereas bulk rheology probes the macroscopic properties of the network, at the millimeter to centimer scale, dynamic light scattering allows us to measure local fluctuations of the network at the length scale of the wavelength 
of light, i.e. a few tens to hundreds of nanometers. With DLS, fluctuations arising for example from the diffusive motion of polymer chains or clusters, collective viscoelastic relaxations in the network or local vibrations in glassy solids, can be determined accurately ${ }^{[77-79]}$. Although various dynamic light scattering studies on transient gels have been reported ${ }^{[78,80-85]}$, the interpretation of the various observed modes in terms of microscopic behaviors remains elusive.

\subsection{Gelation phenomenon}

In the previous paragraph we described the stages of structure formation in telechelic polymer systems en-route to gelation. When approaching the percolation threshold, both the structure of the connected clusters, and the associated relaxation mechanisms, reach a fractal state where no characteristic length- or time-scales can be identified. This scale-free structure and dynamics at the microscopic scale, results in a power-law behavior of the complex modulus at the macroscopic scale, as $G^{*}(\omega)=(i \omega)^{\alpha}$ over a wide range of frequencies ${ }^{[86]}$. The value of the critical scaling exponent $\alpha$ differs from material to material and usually ranges from 0.1 to 0.8 for near-critical polymer networks at their percolation point ${ }^{[70]}$. Above the percolation threshold, however, most selfassembled gels show a characteristic length scale set by the distance between crosslinks and a time-scale prescribed by the thermally-activated bond rupture rate; this results in close-to ideal Maxwellian behavior. The plateau value of the storage modulus $G^{\prime}$ at high frequencies obeys classical rubber theory as expressed in the paragraph above. The relaxation of the network in this case, exhibits the aforementioned Arrhenius behavior $\tau_{0} \propto \exp \left(E_{A} / k_{B} T\right)$ where $\tau_{0}$ is determined by a well-defined characteristic time of thermally-activated dissociation of chain ends from the nodes. The activation barrier $E_{A}$ equals the energy difference between a bonded and a non-bonded chain end. Surprisingly, some telechelic networks appear to become kinetically trapped in their critical gel state and continue to exhibit power-law mechanics over a wide range of concentrations at and well above the percolation point ${ }^{[70,74]}$. The origin of this robust critical gel state remains poorly understood. 


\subsection{Composites}

Some of the most promising applications of hydrogels are in the biomedical field. Due to their high compliance, high water-content and biocompatibility they are promising materials for both pharmaceutical applications and for use in regenerative engineering as cell culture scaffolds. The mechanical properties of conventional hydrogels are, however, still far below the abilities of natural tissues; in particular, while hydrogels can be prepared with low moduli, they typically break at very small deformations. By contrast, nature's hydrogels can be compliant and though at the same time.

To enhance the properties of synthetic hydrogel materials, one may look for examples given by nature, and specifically into the molecular architecture of tough tissues. Many biological hydrogel materials, such as human cartilage, have a complex multi-component structure, combining a network of rigid elements, typically protein-based self-assembled fibers, surrounded by a soft and elastic network of a second type. It has been shown that this combination of two different networks gives these materials superior mechanical properties. This simple concept was translated into a synthetic equivalent in 2003 by Gong et al. in the form of so-called interpenetrating double networks; a discovery which was met with large interest and follow-up in the scientific community. The original design proposed by Gong ${ }^{[21]}$ included a brittle and rigid polyelectrolyte gel of poly(2-acrylamido-2-methylpropanesulfonic acid) (PAMPS) embedded in a matrix of soft and flexible polyacrylamide (PAAm) chains, at a specific ratio. Under deformation, the stiff polyelectrolyte network distributes evenly all applied stresses. And while this network would rupture at small deformations by itself, the interpenetrating architecture of a PAAm network in which the PAMPS is embedded protects the entire double gel from breaking. Eventually, the sample reaches the yielding point, a crack nucleates and the polyelectrolyte network breaks, yet the soft matrix remains intact. In the literature this transfer of failure energy from the main hydrogel to a secondary network is called a sacrificial-network strategy. Composites with this architecture perform exceptionally well, reaching fracture strengths in the range of $10 \mathrm{MPa}$, whereas single components break already in the sub-MPa range. The drawback of this 
concept, however, lies in the permanent nature of these interpenetrating networks; therefore, once damaged, double-networks cannot recover any more. An innovation in the direction of self-healing was brought by Sun and coworkers in $2012^{[25]}$. The sacrificial network was exchanged in this case with a highly concentrated physical network built from calcium crosslinked alginate. After damage of the sacrificial alginate network, the crosslinks can reform so that the original structure can heal, leading to a (partial) recovery of the mechanical properties. Nevertheless, it remains a challenge to design composite hydrogels that are both fully self-healing and exceptionally strong. Other approaches to increase the toughness of hydrogels include composite hydrogels, filled with various types of nanoparticles like inorganic particles, clay platelets or nanocellulose fibers.

\subsection{Outline of this thesis}

This thesis focuses on obtaining a fundamental understanding of the gelation and the dynamics of transiently crosslinked gel networks. To achieve this, we use two types of telechelic polymers, where the assembly is driven either by solvophobic interactions or by metal-ligand coordination bonds. In this research I employ a number of methods, mostly rheology and light scattering.

Chapter 2 shows the results of studies on mixtures of hydrophobically modified mono- and difunctionalized polyethylene glycol (PEG) with the same hydrophilic-lipophilic balance using dynamic light scattering and rheology. The aim of this research is to describe the role of the connectivity of the network by systematically varying the number of bridges, while keeping the other conditions constant. We focus on the macro- and microscopic dynamic properties of these networks.

In Chapter 3 we experimentally demonstrate how the critical gel state in transient polymer networks results from arrested phase separation. Within the same experimental system, we find either homogeneous networks with Maxwellian behavior or spinodal networks with critical gel-like behavior, 
depending on the association strength of the sticky end groups. Our results show how the pathway to network formation may involve kinetically arrested states with critical properties, and explain the observation of disparate mechanical signatures in seemingly similar systems.

Chapter 4 summarizes an extensive rheological study of a supramolecular network formed by terpyridine functionalized water-soluble telechelic polymers. We perform rheology in both linear and non-linear response regimes and by that we define the percolation threshold for gel formation and the character of the sol-critical gel transition. We also investigate the influence of charge ratio. Additionally, we show that the formed critical gel is strongly shear thinning with a tendency for shear banding and edge fracture.

Chapter 5 describes the orthogonal and hierarchical self-assembly of fully transparent and self-healing polymer composites based on non-covalent interactions alone. We employ a strategy based on the spontaneous generation of multivalent nanoparticle tectons, which we subsequently link together into network structures, using metal-coordination linkages. The resulting materials are highly tunable, with moduli and viscosities ranging over many decades, without any additional chemistry, and display rapid, complete and reversible self-healing. 


\subsection{References}

[1] E. O. Polat, O. Balci, N. Kakenov, H. B. Uzlu, C. Kocabas, R. Dahiya, Scientific Reports 2015, 5.

[2] J. Heikenfeld, P. Drzaic, J.-S. Yeo, T. Koch, Journal of the Society for Information Display 2011, 19, 129-156.

[3] M. F. L. De Volder, S. H. Tawfick, R. H. Baughman, A. J. Hart, Science 2013, 339, 535-539.

[4] 2013, 494, 404-404.

[5] J. Fricke, A. Emmerling, Journal of the American Ceramic Society 1992, 75, 2027-2035.

[6] H. Hu, Z. Zhao, W. Wan, Y. Gogotsi, J. Qiu, Adv. Mater. 2013, 25, 2219-2223.

[7] H. Sun, Z. Xu, C. Gao, Adv. Mater. 2013, 25, 2554-2560.

[8] M. Capelot, M. M. Unterlass, F. Tournilhac, L. Leibler, ACS Macro Letters 2012, 1, 789-792.

[9] T. T. H. Pham, F. A. De Wolf, M. A. Cohen Stuart, J. Van Der Gucht, Soft Matter 2013, 9, 8737-8744.

[10] A. M. Kloxin, A. M. Kasko, C. N. Salinas, K. S. Anseth, Science 2009, 324, 59-63.

[11] U. Posadowska, M. Parizek, E. Filova, M. Wlodarczyk-Biegun, M. Kamperman, L. Bacakova, E. Pamula, International Journal of Pharmaceutics 2015, 485, 31-40.

[12] P. Rychter, E. Pamula, A. Orchel, U. Posadowska, M. Krok-Borkowicz, A. Kaps, N. Smigiel-Gac, A. Smola, J. Kasperczyk, W. Prochwicz, P. Dobrzynski, Journal of Biomedical Materials Research - Part A 2015, 103, 3503-3515.

[13] M. K. Włodarczyk-Biegun, M. W. T. Werten, F. A. De Wolf, J. J. J. P. Van Den Beucken, S. C. G. Leeuwenburgh, M. Kamperman, M. A. Cohen Stuart, Acta Biomaterialia 2014, 10, 3620-3629.

[14] M. P. Arnold, A. U. Daniels, S. Ronken, H. A. García, N. F. Friederich, T. Kurokawa, J. P. Gong, D. Wirz, Cartilage 2011, 2, 374-383. 
[15] C. Fan, L. Liao, C. Zhang, L. Liu, Journal of Materials Chemistry B 2013, 1, 4251-4258.

[16] H. J. Kwon, Advances in Materials Science and Engineering 2014, $2014,7$.

[17] P. A. Parmar, L. W. Chow, J.-P. St-Pierre, C.-M. Horejs, Y. Y. Peng, J. A. Werkmeister, J. A. M. Ramshaw, M. M. Stevens, Biomaterials 2015, 54, 213-225.

[18] B. Sharma, S. Fermanian, M. Gibson, S. Unterman, D. A. Herzka, B. Cascio, J. Coburn, A. Y. Hui, N. Marcus, G. E. Gold, J. H. Elisseeff, Science Translational Medicine 2013, 5, 167ra166-167ra166.

[19] K. L. Spiller, S. A. Maher, A. M. Lowman, Tissue Engineering. Part B, Reviews 2011, 17, 281-299.

[20] P. J. Flory, Principles of Polymer Chemistry, Cornell University Press, Ithaca, United States, 1953.

[21] J. P. Gong, Y. Katsuyama, T. Kurokawa, Y. Osada, Adv. Mater. 2003, 15, 1155-1158.

[22] W. H. Rombouts, M. Colomb-Delsuc, M. W. T. Werten, S. Otto, F. A. De Wolf, J. Van Der Gucht, Soft Matter 2013, 9, 6936-6942.

[23] W. H. Rombouts, D. W. De Kort, T. T. H. Pham, C. P. M. Van Mierlo, M. W. T. Werten, F. A. De Wolf, J. Van Der Gucht, Biomacromolecules 2015, 16, 2506-2513.

[24] W. H. Rombouts, M. Giesbers, J. Van Lent, F. A. De Wolf, J. Van Der Gucht, Biomacromolecules 2014, 15, 1233-1239.

[25] J.-Y. Sun, X. Zhao, W. R. K. Illeperuma, O. Chaudhuri, K. H. Oh, D. J. Mooney, J. J. Vlassak, Z. Suo, 2012, 489, 133-136.

[26] T. Ueno, K. Bundo, Y. Akagi, T. Sakai, R. Yoshida, Soft Matter 2010, 6, 6072-6074.

[27] R. Yoshida, T. Sakai, Y. Hara, S. Maeda, S. Hashimoto, D. Suzuki, Y. Murase, Journal of Controlled Release 2009, 140, 186-193.

[28] R. Yoshida, T. Ueki, 2014, 6, e107.

[29] R. F. Shepherd, F. Ilievski, W. Choi, S. A. Morin, A. A. Stokes, A. D. Mazzeo, X. Chen, M. Wang, G. M. Whitesides, Proceedings of the National Academy of Sciences 2011, 108, 20400-20403. 
[30] E. Wang, M. S. Desai, S.-W. Lee, Nano Letters 2013, 13, 2826-2830.

[31] M. D. Golinska, T. T. H. Pham, M. W. T. Werten, F. A. de Wolf, M. A. Cohen Stuart, J. van der Gucht, Biomacromolecules 2013, 14, 4855 .

[32] M. D. Golinska, M. K. Włodarczyk-Biegun, M. W. T. Werten, M. A. C. Stuart, F. A. De Wolf, R. De Vries, Biomacromolecules 2014, 15, 699-706.

[33] S. Coulibaly, A. Roulin, S. Balog, M. V. Biyani, E. J. Foster, S. J. Rowan, G. L. Fiore, C. Weder, Macromolecules 2014, 47, 152-160.

[34] T. Rossow, S. Seiffert, in Supramolecular Polymer Networks and Gels, Vol. 268 (Ed.: S. Seiffert), Springer International Publishing, 2015, pp. $1-46$.

[35] C. Ligoure, S. Mora, Rheol Acta 2013, 52, 91-114.

[36] L. Ramos, A. Laperrousaz, P. Dieudonné, C. Ligoure, Physical Review Letters 2011, 107, 148302.

[37] Y. Séréro, V. Jacobsen, J. F. Berret, R. May, Macromolecules 2000, 33, 1841-1847.

[38] P. J. Skrzeszewska, F. A. de Wolf, M. W. T. Werten, A. P. H. A. Moers, M. A. Cohen Stuart, J. van der Gucht, Soft Matter 2009, 5, 2057-2062.

[39] J.-M. Lehn, Proceedings of the National Academy of Sciences 2002, 99, 4763-4768.

[40] A. Wiseman, Journal of Chemical Technology 8 Biotechnology 1995, 63, 195-195.

[41] F. Laflèche, D. Durand, T. Nicolai, Macromolecules 2003, 36, 13311340 .

[42] F. Laflèche, T. Nicolai, D. Durand, Y. Gnanou, D. Taton, Macromolecules 2003, 36, 1341-1348.

[43] M. Lemmers, J. Sprakel, I. K. Voets, J. van der Gucht, M. A. Cohen Stuart, Angewandte Chemie International Edition 2010, 49, 708-711.

[44] T. T. H. Pham, J. Wang, M. W. T. Werten, F. Snijkers, F. A. de Wolf, M. A. Cohen Stuart, J. van der Gucht, Soft Matter 2013, 9, 8923-8930.

[45] P. J. Skrzeszewska, J. Sprakel, F. A. de Wolf, R. Fokkink, M. A. Cohen Stuart, J. van der Gucht, Macromolecules 2010, 43, 3542-3548. 
[46] J. Sprakel, E. Spruijt, M. A. Cohen Stuart, N. A. M. Besseling, M. P. Lettinga, J. van der Gucht, Soft Matter 2008, 4, 1696-1705.

[47] T. Annable, R. Buscall, R. Ettelaie, D. Whittlestone, Journal of Rheology 1993, 37, 695-726.

[48] N. Cathébras, A. Collet, M. Viguier, J.-F. Berret, Macromolecules 1998, 31, 1305-1311.

[49] A. N. Semenov, J. F. Joanny, A. R. Khokhlov, Macromolecules 1995, 28, 1066-1075.

[50] T. Uneyama, S. Suzuki, H. Watanabe, Physical Review E 2012, 86, 031802 .

[51] J. Sprakel, N. A. M. Besseling, M. A. Cohen Stuart, F. A. M. Leermakers, The European Physical Journal E 2008, 25, 163-173.

[52] Q. T. Pham, W. B. Russel, J. C. Thibeault, W. Lau, Macromolecules 1999, 32, 2996-3005.

[53] Q. T. Pham, W. B. Russel, J. C. Thibeault, W. Lau, Macromolecules 1999, 32, 5139-5146.

[54] J.-F. Berret, Y. Séréro, B. Winkelman, D. Calvet, A. Collet, M. Viguier, Journal of Rheology 2001, 45, 477-492.

[55] J. P. Kaczmarski, J. E. Glass, Macromolecules 1993, 26, 5149-5156.

[56] B. G. G. Lohmeijer, U. S. Schubert, Macromolecular Chemistry and Physics 2003, 204, 1072-1078.

[57] R. Wang, M. Geven, P. J. Dijkstra, P. Martens, M. Karperien, Soft Matter 2014, 10, 7328-7336.

[58] T. Rossow, A. Habicht, S. Seiffert, Macromolecules 2014, 47, 64736482 .

[59] W. Weng, J. B. Beck, A. M. Jamieson, S. J. Rowan, Journal of the American Chemical Society 2006, 128, 11663-11672.

[60] H. P. van Leeuwen, W. Koster, Physiochemical Kinetics and Transport at Biointerfaces, Vol. 9, John Wiley \& Sons, 2004.

[61] M. C. Aragoni, M. Arca, A. Bencini, A. J. Blake, C. Caltagirone, G. De Filippo, F. A. Devillanova, A. Garau, T. Gelbrich, M. B. Hursthouse, F. Isaia, V. Lippolis, M. Mameli, P. Mariani, B. Valtancoli, C. Wilson, Inorganic Chemistry 2007, 46, 4548-4559. 
[62] C. Goze, G. Ulrich, L. Charbonnière, M. Cesario, T. Prangé, R. Ziessel, Chemistry - A European Journal 2003, 9, 3748-3755.

[63] Z. Liu, W. He, Z. Guo, Chemical Society Reviews 2013, 42, 1568-1600.

[64] S. Bode, L. Zedler, F. H. Schacher, B. Dietzek, M. Schmitt, J. Popp, M. D. Hager, U. S. Schubert, Adv. Mater. 2013, 25, 1634-1638.

[65] G. Hong, H. Zhang, Y. Lin, Y. Chen, Y. Xu, W. Weng, H. Xia, Macromolecules 2013, 46, 8649-8656.

[66] J. M. J. Paulusse, R. P. Sijbesma, Angewandte Chemie International Edition 2004, 43, 4460-4462.

[67] A. Winter, G. R. Newkome, U. S. Schubert, ChemCatChem 2011, 3, 1384-1406.

[68] H. Hofmeier, U. S. Schubert, Chemical Society Reviews 2004, 33, 373399 .

[69] P. K. Iyer, J. B. Beck, C. Weder, S. J. Rowan, Chemical Communications 2005, 319-321.

[70] T. Vermonden, N. A. M. Besseling, M. J. van Steenbergen, W. E. Hennink, Langmuir 2006, 22, 10180-10184.

[71] T. Vermonden, J. van der Gucht, P. de Waard, A. T. M. Marcelis, N. A. M. Besseling, E. J. R. Sudhölter, G. J. Fleer, M. A. Cohen Stuart, Macromolecules 2003, 36, 7035-7044.

[72] J. Wang, R. H. M. de Kool, A. H. Velders, Langmuir 2015, 31, 1225112259 .

[73] T.-A. Asoh, H. Yoshitake, Y. Takano, A. Kikuchi, Macromolecular Chemistry and Physics 2013, 214, 2534-2539.

[74] T. Rossow, S. Seiffert, Polymer Chemistry 2014, 5, 3018-3029.

[75] J. Brassinne, F. D. Jochum, C.-A. Fustin, J.-F. Gohy, International Journal of Molecular Sciences 2015, 16, 990-1007.

[76] X.-X. Meng, W. B. Russel, Journal of Rheology 2006, 50, 169-187.

[77] F. Brochard, P. G. de Gennes, Macromolecules 1977, 10, 1157-1161.

[78] U. Genz, Macromolecules 1994, 27, 3501-3512.

[79] T. Nicolai, W. Brown, S. Hvidt, K. Heller, Macromolecules 1990, 23, 5088-5096. 
[80] C. Charbonneau, C. Chassenieux, O. Colombani, T. Nicolai, Physical Review E 2013, $8 \%$.

[81] A. Duri, L. Cipelletti, EPL (Europhysics Letters) 2006, 76, 972.

[82] A. H. a. W. Krall, D. A., Physical Review Letters 1998, 80.

[83] J. Li, T. Ngai, C. Wu, Polymer 2010, 42, 609-625.

[84] E. Michel, L. Cipelletti, E. d'Humieres, Y. Gambin, W. Urbach, G. Porte, J. Appell, Physical Review E 2002, 66, 031402

[85] M. Schwab, B. Stühn, The Journal of Chemical Physics 2000, 112, 6461-6471.

[86] H. Winter, M. Mours, in Neutron Spin Echo Spectroscopy Viscoelasticity Rheology, Vol. 134, Springer Berlin Heidelberg, 1997, pp. 165-234. 
CHAPTER 2

\section{Multiple relaxation modes in associative polymer networks with varying connectivity}

The dynamics and mechanics of networks depends sensitively on their spatial connectivity. To explore the effect of connectivity on local network dynamics, we prepare transient polymer networks in which we systematically cut connecting bonds. We do this by creating networks formed from hydrophobically modified difunctionalized polyethylene glycol (PEG) chains. These form physical gels, consisting of flowerlike micelles that are transiently crosslinked by connecting bridges. By introducing monofunctionalized chains, we can systematically sever the bonds between micelles and thus lower the network connectivity, which strongly reduces the network elasticity and relaxation time. Dynamic light scattering reveals a complex relaxation dynamics which are not apparent in bulk rheology. We observe three distinct relaxation modes. First we find a fast diffusive mode which does not depend on the number of bridges and is attributed to diffusion of micelles within a cage formed by neighboring micelles. A second, intermediate mode, depends strongly on network connectivity but, surprisingly, is independent of the scattering vector $q$. We attribute this viscoelastic mode to fluctuations in local connectivity of the network. The slowest mode is also diffusive and is attributed to the diffusion of micelle clusters through the viscoelastic matrix. These results shed new light on the microscopic dynamics in weakly-interconnected transient networks.

This chapter was submitted as:

M. Bohdan, J. Sprakel, J. van der Gucht: Multiple relaxation modes in associative polymer networks with varying connectivity. 


\section{$2.1 \quad$ Introduction}

Transient networks of physically-crosslinked polymers embedded in a liquid solvent are an archetypal viscoelastic material ${ }^{[1,2]}$. These networks typically form by self-assembly of polymers that contain self-associating groups at specific locations along the chain backbone. The simplest design of such a network forming polymer is a triblock (ABA) copolymer, with a soluble middle block (B) and associating ends (A). These end blocks could be hydrophobic groups ${ }^{[2-5]}$, ionic blocks that form complexes with oppositely charged polymers ${ }^{[5,6]}$, metalbinding groups ${ }^{[6]}$, or biological self-assembly motifs ${ }^{[7-10]}$. At low concentrations, association of the end blocks leads to the formation of small polymer clusters or flowerlike micelles. Above the overlap concentration of the polymers, bridges form between the micelles resulting in the formation of a transient percolating network with a finite shear modulus and a viscosity that increases strongly with increasing concentration. The viscoelastic properties of the network are controlled by both the lifetime of the bridges in the network, which is governed by thermally-activated end-block exchange, and network topology. Rheological measurements indeed show a direct correlation between the viscoelastic relaxation time as measured with a rheometer and the association strength of the A blocks ${ }^{[10,11]}$. For example, it was shown that for PEO chains modified with two alkyl tails, the viscoelastic relaxation time increases exponentially with the length of the tails ${ }^{[3,4]}$, which can be attributed to an activation energy that increases linearly with alkyl length.

The microscopic dynamics in these transient networks can be probed by dynamic light scattering (DLS). This technique is sensitive to fluctuations in concentration and thus probes the mobility of polymers or clusters of polymers at length scales comparable to $1 / q$, where $q$ is the scattering vector. Although the relaxation dynamics of transient networks has been studied extensively in the last decades, the interpretation of the experimental results remains a subject of debate. The relaxation spectrum of transient networks shows a number of different modes: in addition to a fast, rattling mode, also one or more slow relaxation modes are usually observed. The fast mode is generally attributed to the cooperative diffusion of network strands between two transient crosslinks at 
timescales at which the crosslinks can be assumed to be fixed ${ }^{[12]}$. The origin of the slow mode, however, is still poorly understood ${ }^{[13]}$. Some authors report a slow mode with a $q^{2}$ dependence, which is attributed to the diffusion of aggregates or larger clusters through the viscoelastic matrix ${ }^{[13-16]}$. Others, however, find a $q$-independent slow mode, for which diffusion is not the ratelimiting step. This so-called viscoelastic mode is ascribed to changes in network topology caused by reorganization of the connecting bridges ${ }^{[13,17-20]}$. Other $q$-dependencies were also observed and attributed to ballistic motion due to slow relaxation of internal stresses during ageing ${ }^{[21,22]}$ or to relaxation of fractal aggregates ${ }^{[23]}$.

Why such conflicting experimental results are found and what factors determine the mode that prevails in a particular system remain poorly understood. Here, we shed new light on this question by studying relaxation in networks whose architecture is systematically varied. More specifically, we address the role of the connectivity of the network by systematically varying the number of bridges, while keeping the other conditions constant. We do this by using mixtures of difunctionalized and monofunctionalized polymers (Fig. 2.1): as the fraction of difunctionalized polymers decreases, the number of bridges also decreases. This is equivalent to systematically cutting bonds between neighboring nodes in the network. As shown previously ${ }^{[3,4]}$, this leads to a decrease of the modulus and viscosity of the samples. Here, we study how connectivity affects the relaxation dynamics observed with dynamic light scattering. In our experiments, we observe three relaxation modes, similarly to earlier reports on other transient networks ${ }^{[24,25]}$. The fast diffusive mode is not affected by the connectivity of the network, but the intermediate viscoelastic mode and the slow diffusive mode become much slower as the connectivity increases.

\section{$2.2 \quad$ Materials and methods}

\section{Chemicals}

Methoxyl polyethylene glycol (mPEG-OH) of $10 \mathrm{~kg} / \mathrm{mol}$ was purchased from SunBio. Dihydroxy-terminated polyethylene glycol of $20 \mathrm{~kg} / \mathrm{mol}$, anhydrous 
toluene, octadecyl isocyanate, dibutyltin dilaurate and tetrahydrofuran were purchased from Sigma-Aldrich. Acrodisc $®$ Syringe Filters with $0.45 \mu \mathrm{m}$ Teflon Membrane were purchased from PALL.

\section{Preparation of $\mathrm{C}_{18} \mathrm{PEG}$ and $\mathrm{C}_{18} \mathrm{PEGC}_{18}$}

$\mathrm{C}_{18}-\mathrm{PEG}$ and $\mathrm{C}_{18}-\mathrm{PEG}-\mathrm{C}_{18}$ are synthetized as follows. First, mono- and dihydroxy functionalized derivatives of poly(ethylene glycol) are dissolved in anhydrous toluene. An excess of octadecyl isocyanate and dibutyltin dilaurathe as the catalyst are added and left to react for $48 \mathrm{~h}$ at a temperature of $80^{\circ} \mathrm{C}$. The functionalized polymers are then isolated in two cycles of dissolution in toluene followed by precipitation in low-boiling petroleum ether. Purification is proceeded with dissolution of the product in a mixture of THF and demineralized water and filtration over $0.45 \mu \mathrm{m}$ Acrodisc $($ Syringe Filter. THF is removed in a rotary evaporator, and water is removed through freeze drying.

\section{Sample Preparation}

Functionalized monomers are dissolved in demineralized water and homogenized for 24 hours. We prepare a series of samples at fixed PEO concentration $40 \mathrm{~g} / \mathrm{L}$, varying the molar ratio of triblock copolymer to total polymer $f=c_{t b} / c_{t o t}$ in a range from 1 (fully connected network) to 0 (unconnected micellar solution). To remove dust and aggregates for light scattering experiments, the samples are centrifuged for 8 hours at $4000 \mathrm{~g}$, prior to experiments.

\section{Rheology}

Oscillatory shear measurements are performed in the linear regime on an Anton Paar MRC 501 in strain-controlled mode, using a cone-plate geometry (gap = $0.0048 \mathrm{~mm}$, diameter $=50 \mathrm{~mm}$ ). A solvent trap protects the sample from evaporation during experiments.

\section{Light Scattering}

Multi-angle dynamic light scattering measurements are recorded with an ALV light scattering apparatus equipped with a vertically polarized $632.8 \mathrm{~nm}$ JDSU $430 \mathrm{~N}$ laser and photomultiplier detector. All dynamic (DLS) and static light 
scattering (SLS) experiments are performed using a cylindrical quartz cuvette of $10 \mathrm{~mm}$ diameter, at a temperature of $21 \pm 0.1^{\circ} \mathrm{C}$, over a range of scattering vectors $q=\frac{4 \pi n_{0}}{\lambda} \sin \left(\frac{\theta}{2}\right)$, where $n_{0}$ is the refractive index of the solvent, $\lambda$ is the wavelength of the incident laser in vacuo, and $\theta$ the detection angle.

From the SLS experiment, we calculate the Rayleigh ratio $R_{\theta}$ as an absolute measure for scattered light intensity:

$$
R_{\theta}=\frac{I_{\text {sample }}-I_{\text {solvent }}}{I_{\text {toluene }}} \times R_{\text {toluene }} \times \frac{n_{\text {solvent }}^{2}}{n_{\text {toluene }}^{2}}
$$

where $I_{\text {sample }}, I_{\text {solvent }}$ and $I_{\text {toluene }}$ are the scattering intensities measured for the sample, the solvent and the toluene reference, $R_{\text {toluene }}$ is the Rayleigh ratio of toluene at given angle, $n_{\text {solvent }}$ and $n_{\text {toluene }}$ are refractive indexes of the solvent and toluene. Knowing the Rayleigh ratio of our sample at given concentration $\mathrm{C}$ we are able to estimate the molar mass $\mathrm{M}$ of the scatterers as:

$$
\frac{K_{R} C}{R_{\theta}}=\frac{1}{M} \times \frac{1}{P(q R)} \times \frac{1}{S(q)}
$$

where $R$ is the micelle radius, and $P(q)$ and $S(q)$ are the form and structure factors, respectively. The optical constant $K_{R}$ is defined as: $K_{R}=\frac{4 n_{m}^{2} \pi^{2}}{\lambda^{4} N_{A v}} \times\left(\frac{d n}{d c}\right)^{2}$, where $n_{m}$ is the refractive index of the medium, $\frac{d n}{d c}$ is the refractive index increment of the solute, $\lambda$ is the wavelength of the laser and $N_{A v}$ is Avogadro's number. For small particles at low concentrations, we can assume that $P(q R)=1$ and $\frac{1}{S(q)}=1+2 B_{2} \frac{C}{M}$, in which $B_{2}$ is the second virial coefficient describing pair interactions between scatterers, here the flowerlike micelles. From this we can derive that:

$$
\frac{K_{R} C}{R_{\theta}}=\frac{1}{M}+2 B_{2} \frac{C}{M^{2}}
$$

By plotting $\frac{K_{R} C}{R_{\theta}}$ versus concentration, we thus obtain both the molar mass $M$ and second viral coefficient $B_{2}$ of the micelles. 
In DLS we measure the intensity correlation function, $g_{2}(t)=\langle I(0) I(t)\rangle /\langle I\rangle^{2}$, from which we obtain the normalized field autocorrelation function $g_{1}(t): \quad g_{2}(t)=1+\beta[g 1(t)]^{2}$ where $\beta$ is a constant depending on the experimental geometry. In all samples, we observe a full decay of the correlation curves, indicating that the samples are ergodic. Nevertheless, to obtain good averaging, we perform measurements at different positions in the measuring cuvette for each sample. The normalized field autocorrelation function contains information about the density fluctuations in the system ${ }^{[26]}$

$$
g_{1}(t)=\sum_{j, k}\left\langle\exp \left(i \mathbf{q} \cdot\left[\mathbf{r}_{j}(0)-\mathbf{r}_{k}(t)\right]\right)\right\rangle
$$

We observe three relaxation modes in our experiments, and we fit $g_{1}(t)$ to a sum of three stretched exponentials:

$$
g_{1}(t)=\sum_{i=1}^{3} A_{i} \exp \left(-\frac{t}{\tau_{i}}\right)^{\alpha_{i}}
$$

where $A_{i}, \tau_{i}$, and $\alpha_{i}$ are the amplitude, relaxation time, and stretch exponent of the three modes, respectively, with the constraint that $A_{1}+A_{2}+A_{3}=1$.

\subsection{Results and discussion}

The telechelic polymer used in our studies consists of a hydrophilic $20 \mathrm{kDa}$ poly(ethylene glycol) spacer end-capped with hydrophobic octadecyl functional groups. When dissolved in water, the hydrophobic end groups self-associate, resulting in a distinct structure formation upon increasing the polymer concentration. Above a critical micelle concentration, the polymers self-assemble into flower-like micelles.

We characterize these micelles using dynamic and static light scattering, using a monofunctionalized equivalent of our telechelic polymer (10 kDa poly(ethylene glycol) with one single $\mathrm{C}_{18}$ functionality), at a concentration of $5 \mathrm{~g} / \mathrm{l}$. The absence of difunctional associating polymers ensures the absence of crosslinks 
between the micelles. Moreover, at this low concentration, repulsive interactions between micelles can be neglected, so that the measured diffusion coefficient corresponds to the self-diffusion coefficient of the micelles. We find a hydrodynamic radius $R_{h}$ of the micelles of $16 \mathrm{~nm}$. From the static light scattering experiments we determine a molar mass per micelle of $526 \mathrm{~kg} / \mathrm{mol}$, which corresponds to an aggregation number of $p \approx 53$ associative ends per micelle. These values are in good agreement with literature ${ }^{[3,4,27,28]}$.

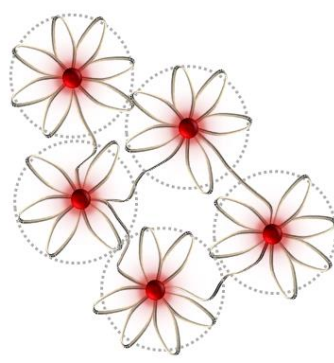

$f=1$

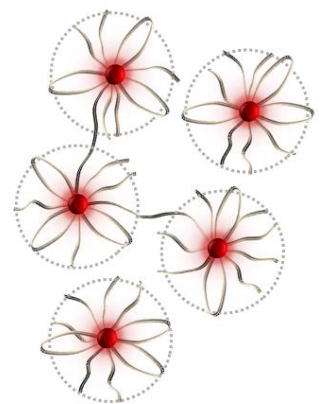

decreasing $f$



$f=0$

Figure 2.1: Schematic illustration of a telechelic associating polymer solution above the critical micelle concentration ( $\mathrm{cmc}$ ) with decreasing difunctionalized chain ratio. Upon decreasing the functionality $f$, the number of bridges decreases and at $f=0$ the solution consists of densely packed non-attached polymeric micelles.

At higher polymer concentrations, the micelles begin to interact. For triblock copolymers, bridging causes an entropic attraction between the micelles. Above a critical concentration, this attraction can lead to macroscopic phase separation of the solution into a dense phase of interconnected micelles and a dilute phase consisting of free micelles and unimers ${ }^{[2,29,30]}$. The two-phase region ends when the solution crosses the binodal concentration, here observed approximately at $15 \mathrm{~g} / \mathrm{L}$. For concentrations above $15 \mathrm{~g} / \mathrm{L}$, the entire solution consists of a homogeneous sample-spanning network of transiently connected micelles. The phase behavior of similar systems was studied extensively by various groups as 
a function of temperature, concentration, functionality, or added surfactant ${ }^{[3,4}$, $27,28,31]$.

Here, we will focus on the relaxation dynamics in the network phase. We therefore choose a polymer concentration of $40 \mathrm{~g} / \mathrm{L}(2 \mathrm{mM})$, which is well above the binodal concentration. From the values for the micellar size and aggregation number obtained above, we estimate the effective volume fraction of micelles to be $\varphi \approx 0.78$. Even though this volume fraction appears to be quite high, the samples with only monofunctionalized polymer do not show significant elasticity. This indicates that the micelles can still move with respect to each other, probably because the outer region of the micellar corona is very dilute and soft. We systematically monitor changes in the relaxation dynamics of such networks in response to changes in their connectivity. This is done by replacing part of the difunctionalized polymer with monofunctionalized polymer of half the chain length (10kDa PEG-spacer), in such a way that the total polymer weight concentration remains the same. This is fully equivalent to cutting bridging chains exactly in half. We define $f$ as the fraction of difunctionalized polymer in the sample, $c_{t b} / c_{\text {tot }}$ which we vary between 1 and 0 , going from a maximally-connected network to a fully unconnected micellar solution.

\subsubsection{Oscillatory shear measurements}

We use oscillatory rheology to probe the frequency-dependence of the linear shear viscoelasticity of our transient networks. The measured curves exhibit a characteristic quasi-Maxwellian shape, known for telechelic associative polymers (Fig.2.2). The crossover of storage and loss moduli (Fig.2.2a) is associated with a characteristic mechanical relaxation time, $\tau_{R}=1 / \omega_{\text {cross. }}$. This time scale can be related to the thermally-activated dissociation of chain ends from micellar nodes which exhibits Arrhenius behavior ${ }^{[3,4]}$. The plateau modulus $G_{0}$, i.e. the high-frequency plateau in $G^{\prime}$, is a measure for the network elasticity at short time scales, and thus probes the number of elastically active chains in the network. As expected, we find that the plateau modulus $G_{0}$ decreases strongly as the network connectivity, expressed as the mixing ratio $f$, decreases. This signals the gradual depercolation of the network. 

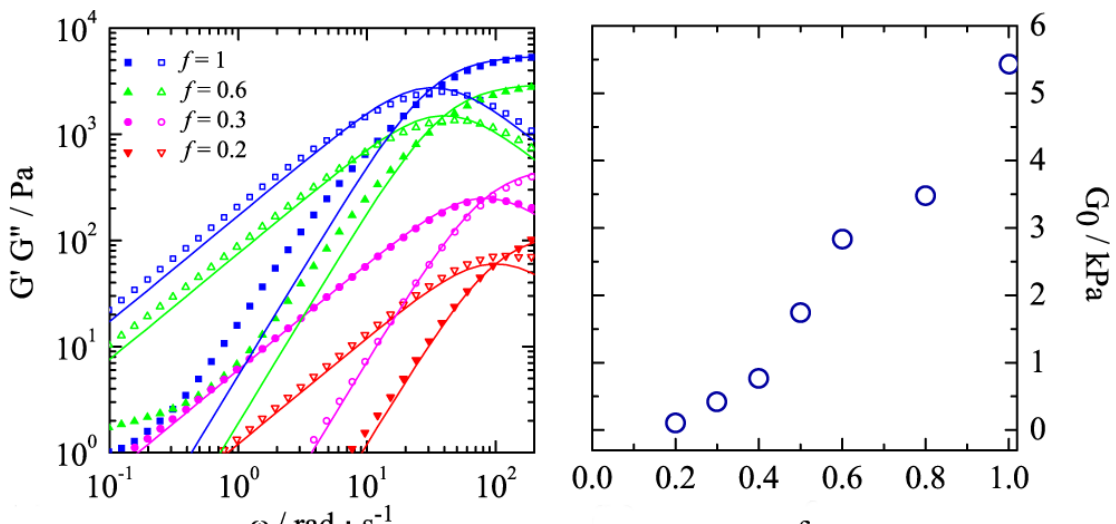

(a)

$\omega / \mathrm{rad} \cdot \mathrm{s}^{-1}$

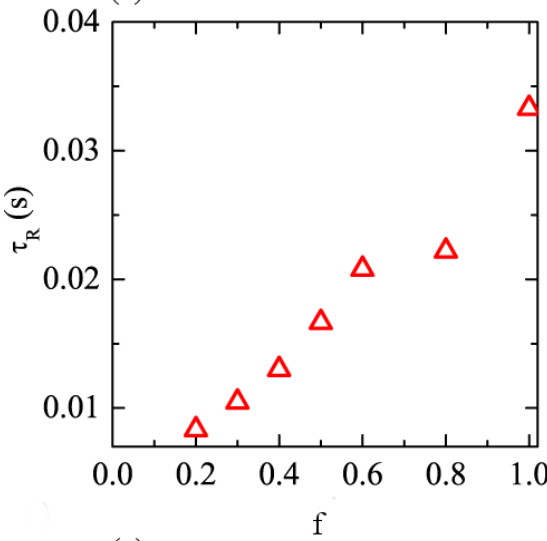

(c)

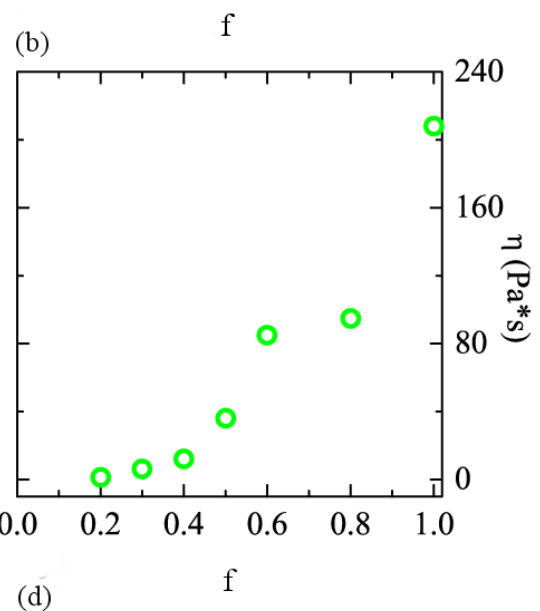

Figure 2.2: Rheological characterization of transient networks. (a) Storage (full symbols) and loss modulus (open symbols) as a function of angular frequency for networks with different functionalities $f$; (b) plateau storage modulus as a function of $f ;(c)$ terminal relaxation time $\tau_{R}$ as a function of $f ;(d)$ zero-shear viscosity as a function of $f$.

The plateau modulus $G_{0}$ drops nearly linearly with decreasing $f$, in agreement with classical network theory, which predicts that $G_{0}$ is proportional to the number of elastically active network chains per unit volume, $G_{0} \approx n k T \sim n_{\text {tot }} f k T^{[20]}$. For the network with $f=1$, we find a plateau modulus of $\sim 5.5 \mathrm{kPa}$, which is very close to the prediction of classical network theory assuming that all chains are elastically active at this micelle volume fraction. As shown in Fig.2.2c and Fig.2.2d, the terminal relaxation time and the viscosity 
also decrease strongly as the network connectivity is reduced. This suggests that not only thermally-activated node dissociation governs the mechanical relaxation, but that network topology also plays a crucial role. This can be ascribed to the presence of super-bridges in the diluted networks, consisting of strands of several micelles that are connected to only two other micelles ${ }^{[9,11]}$. In these superbridges, composed of multiple nodes, each of which can dissociate, relaxation is enhanced as only one of the junctions needs to be broken for the stress carried by the bridge to be relaxed.

\subsubsection{Dynamic light scattering measurements}

To probe the microscopic relaxation dynamics in the transient networks, we perform dynamic light scattering on the samples; we measure the intensity autocorrelation functions for samples with varying connectivity as shown in Fig. 2.3a. It is clear that none of the recorded curves shows a simple exponential decay. Rather, we observe three relaxation modes, in agreement with earlier studies on different transient networks ${ }^{[24,25]}$. To extract the characteristic relaxation times and amplitudes for each of these modes, we fit the autocorrelation curves to equation (5). Below, we discuss the three relaxation modes, and their dependence on the fraction of difunctional polymers $f$.

\section{The fast relaxation mode}

The fastest mode can be fitted with a simple exponential decay in all cases, i.e. its stretch exponent $\alpha_{f}=1$. This is indicative of a relaxation process with a well-defined characteristic relaxation time $\tau_{f}$ (Fig.2.3b). We find that this characteristic time is virtually constant as the fraction of difunctionalized polymer $f$ is changed (squares in Fig.2.3b). This implies that the fast relaxation process does not depend significantly on the connectivity of the network. We attribute the fast relaxation mode to the collective diffusion of micelles over short distances, at time scales where the network topology is fixed ${ }^{[12]}$. 


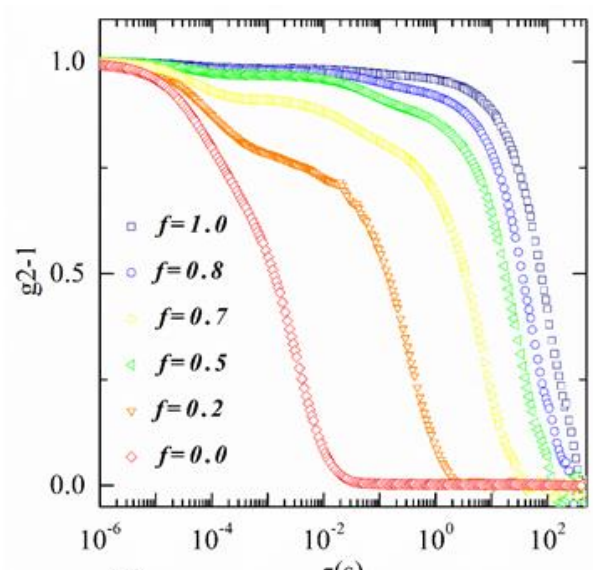

(a)

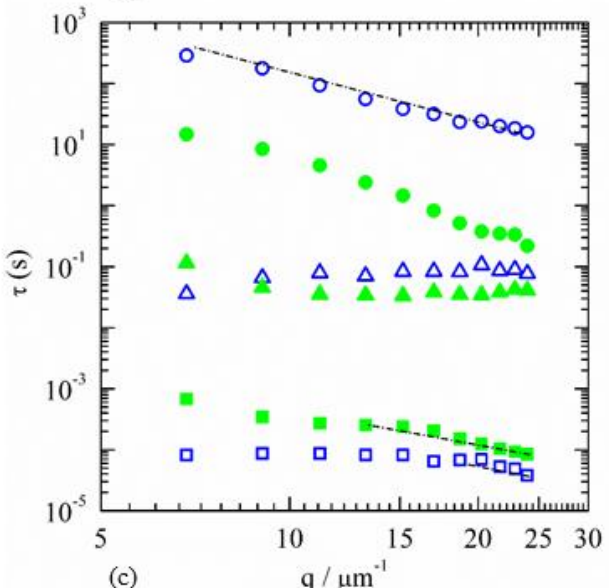

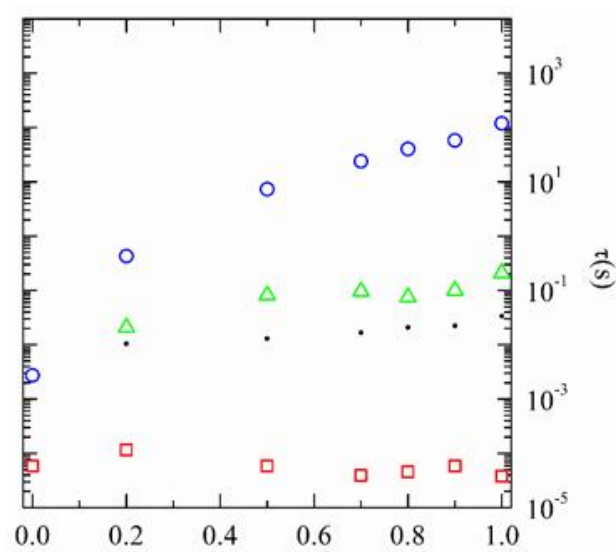

(b)

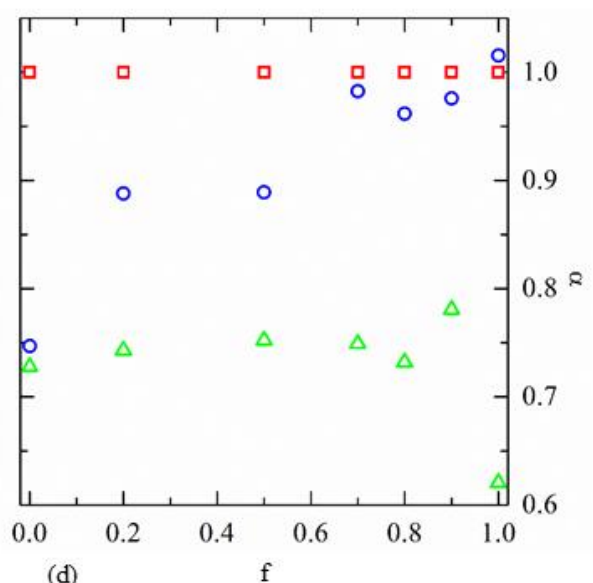

(d)

Figure 2.3 (a) Autocorrelation curves measured using dynamic light scattering for transient networks with several $f$; (b) fast (red squares), intermediate (green triangles), and slow (blue circles) relaxation times, obtained by fitting the autocorrelation curves to equation (5), as a function of $f\left(q=0,018 \mathrm{~nm}^{-1}\right)$; (c) relaxation times as a function of scattering vector $q$ for network with $f=0.5$. for two concentration: $20 \mathrm{~g} / \mathrm{l}$ (magenta diamonds, green triangle, cyan circles) and $40 \mathrm{~g} / \mathrm{l}$ (red squares, olive triangles, blue circles) (d) stretch exponents of the three different modes as a function of functionality $f$. 
The collective diffusion coefficient is proportional to the osmotic compressibility of the network, which is determined by the polymer concentration rather than by the network connectivity ${ }^{[32]}$.

As shown in Fig.2.3c, $\tau_{f}$ decreases approximately as $q^{-2}$ at large values of $q$, as expected for a diffusive mode. At low $q$, however, the relaxation time levels off. We have no explanation for this $q$-independent region, but it should be noted that the contribution of the fast mode to the correlation curve is very small at small $q$, where relatively large length scales are probed, so that an accurate determination of $\tau_{f}$ is difficult in this region. From the measured relaxation times in the diffusive regime, we obtain a collective diffusion coefficient $D_{\text {eff }}=7.6 \times 10^{-11} \mathrm{~m}^{2} / \mathrm{s}$, which is about five times higher than the diffusion coefficient of micelles in dilute solution, $D_{0}=1.6 \times 10^{-11} \mathrm{~m}^{2} / \mathrm{s}$. Such an increase of the collective diffusion coefficient at high concentration is commonly observed and is attributed to the increase in osmotic pressure which speeds up density fluctuations ${ }^{[33]}$.

\section{The intermediate relaxation mode}

The second mode, which we will refer to as the intermediate mode, has very different signatures. It shows a stretched exponential decay, with stretch exponent $\alpha_{i} \approx 0.7$, which is an indication of heterogeneous dynamics (Fig.2.3d). As shown in Fig.2.3c, the mean relaxation time associated with this mode is independent of the scattering vector $q$. Such a non-diffusive mode has been observed before in semi-dilute polymer solutions and in transient polymer networks ${ }^{[13,17-20]}$, and is often referred to as a viscoelastic mode. Its occurrence can be explained using a viscoelastic two-fluid model, which accounts for the interplay between mutual diffusion and viscoelastic relaxations ${ }^{[12,34,35]}$. For systems with a rheological relaxation time $\tau_{R}$ that is large $\left(\tau_{R} \gg D_{e f f} q^{2}\right)$, these models predict the presence of a relaxation mode for which diffusion is not the rate-limiting step.

The characteristic time of this mode is determined by reorganizations of the transient nodes in the network and should be proportional to the rheological relaxation time $\tau_{R}{ }^{[35]}$. Fig. $2.3 \mathrm{~b}$ shows that the intermediate relaxation time 
increases strongly as the fraction of bridges in the network increases, from approximately $0.02 \mathrm{~s}$ at $f=0.2$ to approximately $0.2 \mathrm{~s}$ at $f=1$.

This increase in relaxation time is indeed similar to the increase in the mechanical relaxation time as determined from bulk rheology (black dots in Fig.2.3b), although the absolute values are about a factor 3-5 larger. Note that for the sample with only monofunctional polymers only two modes can be discerned. No viscoelastic mode is observed in this case, as there are no transient crosslinks in the absence of difunctionalized polymer.

\section{The slow relaxation mode}

The slowest mode in the relaxation spectrum is a diffusive mode, exhibiting a $q^{-2}$ dependence (Fig.2.3c). Its mean relaxation time increases very strongly with increasing $f$ (circles Fig.2.3b). For large values of $f$, the slow mode can be fitted with a stretch exponent close to unity (Fig,2.4d). However, for $f<0.5$ its nature changes and the decay becomes a stretched exponential, with a stretch exponent $\alpha_{s} \approx 0.8$. This indicates that the dynamics become more heterogeneous as the connectivity is reduced and the system approaches its percolation transition. The diffusive nature of the slow mode suggests that this mode is due to the long-time diffusion of micelles, or micelle clusters through the viscoelastic matrix, with an effective diffusion coefficient $D_{s}=1 / q^{2} \tau_{s}$.



Figure 2.4: Changes in effective diffusion coefficient compared with the changes in viscosity of the sample with varied connectivity. 
According to the Stokes-Einstein relation, we expect this diffusion coefficient to be inversely proportional to the viscosity of the sample. To test this, we calculate the ratio between the diffusion coefficient corresponding to the slow mode and the diffusion coefficient of micelles in dilute solution, $D_{0}=1.6 \times 10^{-11} \mathrm{~m}^{2} / \mathrm{s}$. In Fig.2.4, we compare this ratio $\left(D_{0} / D_{s}\right)$ to the viscosity ratio $\eta_{0} / \eta$, with $\eta_{0}$ the viscosity of the solvent and $\eta$ the viscosity of the sample. For low connectivity, the two ratios nearly coincide, indicating that the relaxation rate of the slow mode is indeed limited by diffusion of micelles in this regime of weakly connected micelles. However, at larger values of $f$, we see that the long-time diffusion coefficient decreases more strongly than expected on the basis of the macroscopic viscosity. This suggests that for higher functionalities the diffusing objects are clusters or aggregates of micelles that are much larger than a single micelle.

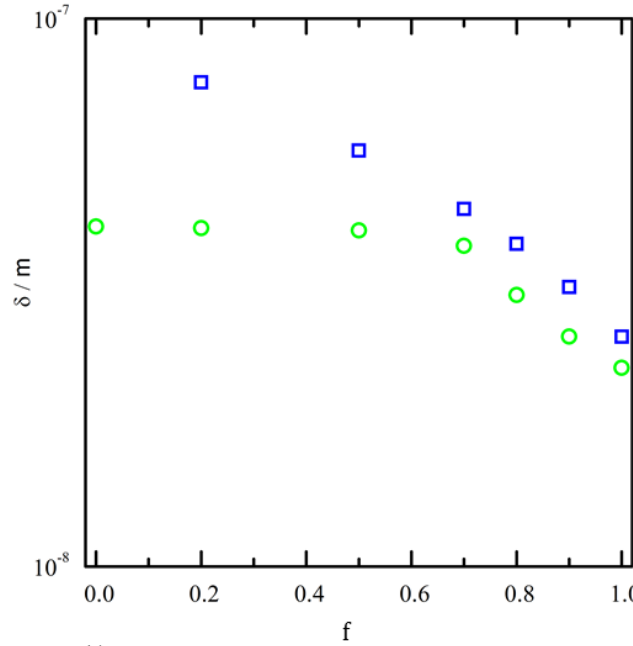

(a)

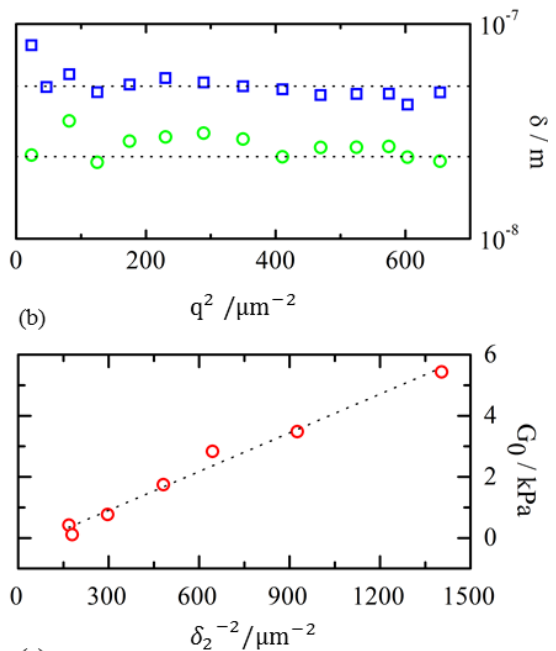

(c)

Figure 2.5: The effective displacement of the micelles after full relaxation of the fast (green circles) and intermediate mode (blue squares) (a) as a function of the functionality ratio $f$, and (b) as a function of $q^{2}$. (c) Plateau modulus as a function of $1 / q^{2}$.

Finally, we consider the amplitudes $A_{i}$ of the different modes. For micelles that are separated by a distance larger than $q^{-1}$, the contribution of terms with 
$j \neq k$ to equation (4) can be neglected ${ }^{[31,36]}$. In that case, the correlation function can be directly related to the mean square displacement of the micelles: $g_{1}(t)=\exp \left(-q^{2}\left\langle\Delta r^{2}(t)\right\rangle / 6\right)$. In our experiments, where $q^{-1}$ is on the order of $50 \mathrm{~nm}$ and the micelle diameter is approximately $30 \mathrm{~nm}$, this approximation is not strictly valid. Nevertheless, we can use the plateaus in $g_{1}(t)$ to obtain a rough estimate for the effective displacement after each relaxation mode: $\delta_{1}^{2}=-6 q^{-2} \ln \left(1-A_{f}\right)$ and $\delta_{2}^{2}=-6 q^{-2} \ln \left(1-A_{f}-A_{\text {int }}\right)$, where $1-A_{f}$ is the value of $g_{1}$ after full relaxation of the fast mode, and $1-A_{f}-A_{\text {int }}$ the value of $g_{1}$ after full relaxation of the intermediate mode. We find that both $\delta_{1}$ and $\delta_{2}$ obtained in this way are independent of $q$ (Fig. 2.5b), so that the $\delta_{i}$ 's can indeed be identified as real displacements. Fig.2.5a shows both $\delta_{1}$ and $\delta_{2}$ as a function of $f$. For low functionalities, the displacements depend only weakly on $f$, and $\delta_{1}$ is on the order of the typical distance between micelles. At larger values of $f$, the plateau displacements decrease, which can be ascribed to an increase of the elasticity of the network, which reduces the fluctuations. Previously, it was predicted that the plateau displacement of a spherical particle in an elastic medium can be related to the elastic modulus as $\delta_{2}^{2} \approx k T / 6 \pi R G_{0}{ }^{[37]}$. Indeed, Fig. $2.5 \mathrm{c}$ shows that the elastic modulus measured with rheology is proportional to $1 / \delta_{1}^{2}$. However, the resulting slope is two orders of magnitude smaller than expected, indicating that the microscopic modulus experienced by the micelles is much smaller than the macroscopic modulus. This was also observed before for similar systems ${ }^{[38,39]}$.

\subsection{Conclusions}

We have studied the relaxation processes of a transient network built from telechelic octadecyl end-capped poly(ethylene glycol) with varying connectivity. Transient networks have been studied using dynamic light scattering before, but the interpretation of the different relaxation modes that can be observed remains a subject of debate. Here, we find that the relaxation spectrum is characterized by three different modes: one fast diffusive mode, an intermediate non-diffusive mode, and a second, slow diffusive mode. By 
systematically varying the number of bridges in the network, while keeping the volume fraction of micelles the same, we can disentangle the effects of concentration and connectivity on the dynamics. The fast mode is hardly affected by the number of bridges, and can be attributed to the cooperative diffusion of micelles at distances where bridges are irrelevant. The other two modes, by contrast, depend strongly on the connectivity; the intermediate mode can be related to the rearrangement of bridges, with a time scale that is proportional to the rheological relaxation time, and the slow diffusive mode is ascribed to the diffusion of micelles or clusters of micelles through the viscoelastic matrix. 


\subsection{References}

[1] S. Seiffert, Supramolecular Polymer Networks and Gels, Vol. 268, 2015.

[2] J. Sprakel, E. Spruijt, M. A. Cohen Stuart, N. A. M. Besseling, M. P. Lettinga, J. van der Gucht, Soft Matter 2008, 4, 1696-1705.

[3] F. Laflèche, D. Durand, T. Nicolai, Macromolecules 2003, 36, 13311340 .

[4] F. Laflèche, T. Nicolai, D. Durand, Y. Gnanou, D. Taton, Macromolecules 2003, 36, 1341-1348.

[5] M. Lemmers, J. Sprakel, I. K. Voets, J. van der Gucht, M. A. Cohen Stuart, Angewandte Chemie International Edition 2010, 49, 708-711.

[6] M. Gerth, M. Bohdan, R. Fokkink, I. Voets, J. van der Gucht, J. Sprakel, Macromolecular Rapid Communications 2014, 35, 2065-2070.

[7] M. D. Golinska, T. T. H. Pham, M. W. T. Werten, F. A. de Wolf, M. A. Cohen Stuart, J. van der Gucht, Biomacromolecules 2013, 14, 4855.

[8] T. T. H. Pham, J. Wang, M. W. T. Werten, F. Snijkers, F. A. de Wolf, M. A. Cohen Stuart, J. van der Gucht, Soft Matter 2013, 9, 8923-8930.

[9] P. J. Skrzeszewska, F. A. de Wolf, M. W. T. Werten, A. P. H. A. Moers, M. A. Cohen Stuart, J. van der Gucht, Soft Matter 2009, 5, 2057-2062.

[10] P. J. Skrzeszewska, J. Sprakel, F. A. de Wolf, R. Fokkink, M. A. Cohen Stuart, J. van der Gucht, Macromolecules 2010, 43, 3542-3548.

[11] T. Annable, R. Buscall, R. Ettelaie, D. Whittlestone, Journal of Rheology 1993, 37, 695-726.

[12] F. Brochard, P. G. de Gennes, Macromolecules 1977, 10, 1157-1161.

[13] J. Li, T. Ngai, C. Wu, Polymer 2010, 42, 609-625.

[14] C. Konak, G. Fleischer, Z. Tuzar, R. Bansil, Journal of Polymer Science Part B: Polymer Physics 2000, 38, 1312-1322.

[15] H. Nie, M. Li, R. Bansil, Č. Koňák, M. Helmstedt, J. Lal, Polymer 2004, 45, 8791-8799.

[16] E. Raspaud, D. Lairez, M. Adam, Macromolecules 1995, 28, 927-933.

[17] M. Doi, A. Onuki, J. Phys. II France 1992, 2, 1631-1656.

[18] U. Genz, Macromolecules 1994, 27, 3501-3512.

[19] E. Michel, M. Filali, R. Aznar, G. Porte, J. Appell, Langmuir 2000, 16, 8702-8711.

[20] T. Nicolai, W. Brown, S. Hvidt, K. Heller, Macromolecules 1990, 23, 5088-5096. 
[21] C. Charbonneau, C. Chassenieux, O. Colombani, T. Nicolai, Physical Review E 2013, 8\%.

[22] A. Duri, L. Cipelletti, EPL (Europhysics Letters) 2006, 76, 972.

[23] M. C. Blanco, D. Leisner, C. Vázquez, M. A. López-Quintela, Langmuir 2000, 16, 8585-8594.

[24] E. Michel, L. Cipelletti, E. d'Humieres, Y. Gambin, W. Urbach, G. Porte, J. Appell, Physical Review E 2002, 66, 031402

[25] M. Schwab, B. Stühn, The Journal of Chemical Physics 2000, 112, 6461-6471.

[26] P. N. Pusey, R. J. A. Tough, Advances in Colloid and Interface Science 1982, 16, 143-159.

[27] Q. T. Pham, W. B. Russel, J. C. Thibeault, W. Lau, Macromolecules 1999, 32, 2996-3005.

[28] Q. T. Pham, W. B. Russel, J. C. Thibeault, W. Lau, Macromolecules 1999, 32, 5139-5146.

[29] A. N. Semenov, J. F. Joanny, A. R. Khokhlov, Macromolecules 1995, 28, 1066-1075.

[30] J. Sprakel, N. A. M. Besseling, M. A. Cohen Stuart, F. A. M. Leermakers, The European Physical Journal E 2008, 25, 163-173.

[31] A. H. a. W. Krall, D. A., Physical Review Letters 1998, 80.

[32] P. G. de Gennes, Scaling Concepts in Polymer Physics, Cornell University Press, Ithaca, 1979.

[33] U. Zettl, S. T. Hoffmann, F. Koberling, G. Krausch, J. Enderlein, L. Harnau, M. Ballauff, Macromolecules 2009, 42, 9537-9547.

[34] F. Brochard-Wyart, Journal de Physique 1983, 44, 39.

[35] E. Michel, G. Porte, L. Cipelletti, J. Appell, Langmuir 2004, 20, 984990.

[36] P. N. Pusey, Physica A: Statistical Mechanics and its Applications 1989, 157, 705-741.

[37] T. G. a. W. Mason, D. A., Physical Review Letters 1995, 74, 12501253 .

[38] J. van der Gucht, N. A. M. Besseling, W. Knoben, L. Bouteiller, M. A. Cohen Stuart, Physical Review E 2003, 67, 051106.

[39] J. Sprakel, J. van der Gucht, M. A. Cohen Stuart, N. A. M. Besseling, Physical Review E 2008, 77, 061502 


\subsection{Appendix}


Fig.A1. Autocorrelation curves measured using dynamic light scattering for transient networks in two concentrations: a) $20 \mathrm{~g} / \mathrm{l}$ and b) $40 \mathrm{~g} / \mathrm{l}$, as a function of scattering vector $q$ for network with $f=0: 5$. 

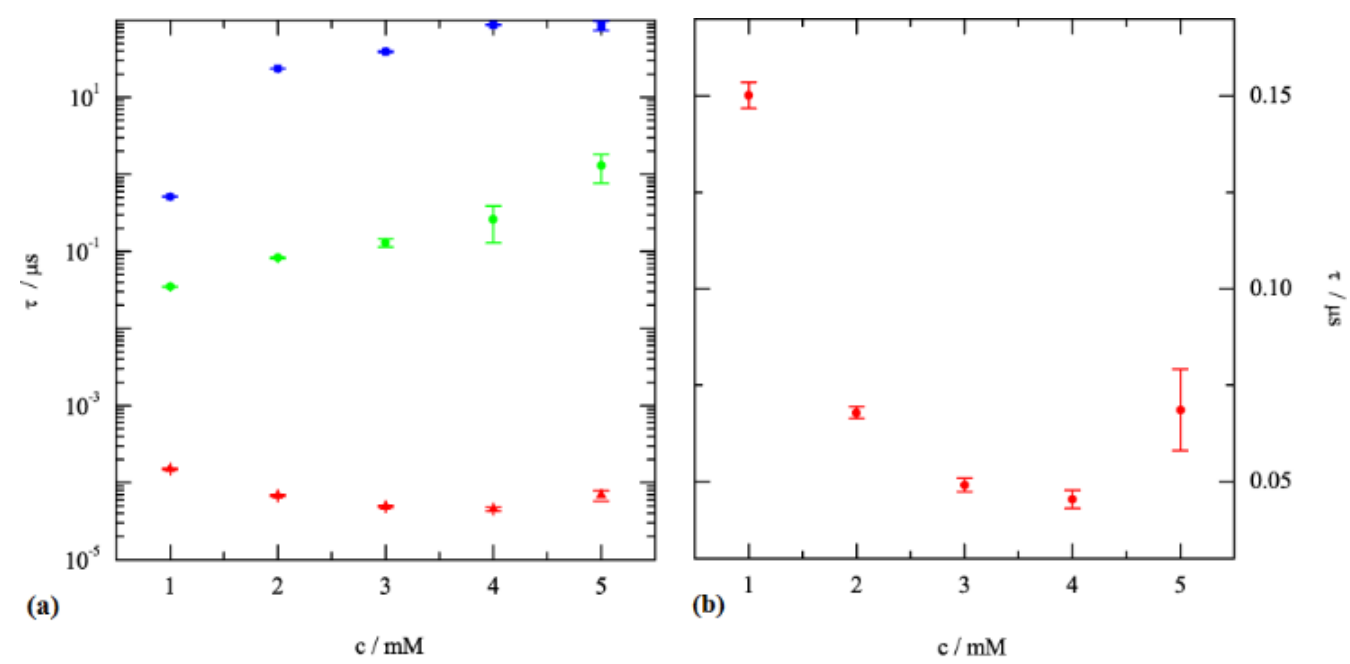

Fig. A2.a) Relaxation times obtained by fitting the autocorrelation curves to equation (5), measured at $q=0,018 \mathrm{~nm}^{-1}$, as a function of overall polymer concentration for $f=0.5, b)$ short relaxation time as a function of polymer concentration. 


\section{Critical polymer networks by arrested phase separation}

Some systems of end-association polymers exhibit a peculiarly robust critical state characterized by scale-invariant linear mechanics that deviates strongly from the expected Maxwellian response. The origins of such a critical state in self-assembling polymer systems remain unclear. Here we show that it results from arrested phase separation, which traps the network-forming system at its percolation point. The emergence of this critical state, and its lifetime, can be controlled by the association strength of polymer ends alone. These results highlight an unexpected pathway to network formation characterized by longlived yet transient arrested states.

This chapter is a part of the manuscript:

M. Bohdan, J. van der Gucht, J. Sprakel: Critical polymer networks by arrested phase separation 


\subsection{Introduction}

Self-assembled polymeric networks, crosslinked with non-permanent nodes, form a versatile class of macro-molecular materials, which exhibit unusual properties, such as self-healing ${ }^{[1]}$, due to the continuous reconfiguration of the structure which is absent in chemically-bonded gels. Transiently-bonded networks can form spontaneously from polymer chains which have selfassociating groups at both chain ends, which organize into crosslinks that remain in a dynamic exchange equilibrium with their surroundings. As the node functionality and its dissociation kinetics can be tailored through the molecular design, the properties of these networks can be finely tuned. This has allowed careful investigation of the effects of network topology and relaxation on both linear ${ }^{[2,3]}$ and non-linear network mechanics ${ }^{[4,5]}$. In principle, the linear rheology of these networks can be described by the Maxwellian spring-dashpot model ${ }^{[6]}$. The high-frequency shear modulus $G_{0}$ is set by the number density $n$ of elastically-active chains between nodes $G_{0} \propto n k B T$. Relaxation of the network occurs via thermally-activated dissociation of chain ends from nodes with a welldefined characteristic time $\tau_{0}$. The relaxation time follows Arrhenius behavior, $\tau_{0} \propto \exp \left(E_{A} / k_{B} T\right)$, with an activation barrier $E_{A}$ equal to the energy difference between a bonded and a non-bonded chain end. Many self-associating telechelic polymer systems indeed exhibit close-to ideal Maxwellian behavior, when entanglements between chains are negligible ${ }^{[3,4,7]}$. Surprisingly, however, certain transient networks manifest completely different properties, where the shear and stress relaxation moduli exhibit distinct power-law scaling with frequency ${ }^{[8,9]}$. The absence of a characteristic modulus and relaxation time in this scaleinvariant mechanics is typically identified only in chemical gels in close proximity to their gel point ${ }^{[10,11]}$. When a crosslinking polymer system approaches its percolation point, both the structure of connected clusters, and the associated relaxation mechanisms, reach a fractal state where no characteristic length or time-scales can be identified. While this critical state only occurs in close proximity to the gel point for chemical gels, this peculiar state appears robustly, e.g. independently of polymer concentration, in certain self-associating polymer systems. It is unclear why the linear mechanics differ 
so strongly between telechelic polymer systems with very similar chemistry. Moreover, the microscopic mechanism with which these systems organize into this robust critical state remains elusive.

In this paper we experimentally demonstrate how the critical gel state in transient polymer networks results from arrested phase separation. Within the same experimental system, we find either homogeneous networks with Maxwellian behavior or spinodal networks with critical gel-like behavior, depending on the association strength of the sticky end groups. Surprisingly, as the supramolecular crosslinks remain transient in all cases, the non-equilibrium critical state slowly evolves into an equilibrated Maxwellian system while the heterogeneous structure slowly vanishes. Our results show how the pathway to network formation may involve kinetically arrested states with critical properties, and explain the observation of disparate mechanical signatures in seemingly similar systems.

\subsection{Methods}

Details regarding materials, synthesis and functionalization assay can be found in Chapter 4.

\section{Sample preparation}

Samples with different concentrations are prepared from stock solutions of $30 \%$ $\mathrm{PEO}(\text { tpy })_{2}, 0.1 \mathrm{M} \mathrm{Ni}\left(\mathrm{NO}_{3}\right)_{2}$ and $\mathrm{FeCl}_{2}, \mathrm{ZnCl}_{2}$ and $\mathrm{CoCl}_{2}$. To improve mixing we add metal solution in a drop-wise manner into an intensively stirred solution of polymer. Unless stated differently samples are mixed vigorously and left for 3 days to equilibrate.

\section{Rheology}

Rheological experiments for all samples are performed with a strain-controlled rheometer (Anton Paar MRC 501), using a cone-plate geometry (gap $=0.0048 \mathrm{~mm}$, diameter $=50 \mathrm{~mm})$. Temperature is controlled by a Peltier system and it is kept constant at $21^{\circ} \mathrm{C}$. To protect samples from drying, the 
geometry is covered with silicon oil as a solvent trap. After loading the samples in the rheometer, they are equilibrated for 10 minutes with constant $1 \%$ oscillations and consecutively left to rest for an additional 10 minutes.

Frequency sweep experiments are performed at 5\% strain in the linear response regime, with decreasing frequency between of 100 and $0.1 \mathrm{rad} / \mathrm{s}$. Stress relaxation moduli are recorded by applying a $5 \%$ constant strain and recording the residual stress.

\section{Static and Dynamic Light Scattering}

Multi-angle dynamic (DLS) and static (SLS) light scattering measurements are recorded with an ALV light scattering apparatus equipped with a vertically polarized $632.8 \mathrm{~nm}$ JDSU 430N laser and photomultiplier detector. All experiments are performed using a cylindrical quartz cuvette of $10 \mathrm{~mm}$ diameter, at a temperature of $21 \pm 0.1^{\circ} \mathrm{C}$, over a range of scattering vectors $q=\frac{4 \pi n_{0}}{\lambda} \sin \left(\frac{\theta}{2}\right)$, where $n_{0}$ is the refractive index of the solvent, $\lambda$ is the wavelength of the incident laser in-vacuo, and $\theta$ the detection angle. All samples used in the light scattering experiments are prepared from solutions filtered with $0.45 \mu \mathrm{m}$ Acrodisc ${ }^{\circledR}$ Syringe Filters. The DLS titration is performed by dropwise addition of $0.1 \mathrm{M} \mathrm{Ni}\left(\mathrm{NO}_{3}\right)_{2}$ to the $1 \mathrm{mM}$ (tpy) $)_{2} \mathrm{PEO}$ solution until the molar ratio of metal $\left[\mathrm{M}^{2+}\right] /\left[(\mathrm{tpy})_{2} \mathrm{PEO}\right]=2$. After each dosage we stir the solution intensively and we measure the scattering intensity and autocorrelation function. Next, we use the CUMULANT method to calculate the hydrodynamic radius:

$$
R_{h}=k T q^{2} / 6 \pi \eta \Gamma
$$

where $q$ is the scattering vector $\left(\sim 0.023 \mathrm{~nm}^{-1}\right), k$ is the Boltzman constant, $T$ is the absolute temperature, $\eta$ is the viscosity of the solvent and $\Gamma$ is the measured average decay rate of the correlation function.

From the multi-angle SLS experiment, we calculate the Rayleigh ratio $R_{\theta}$ as an absolute measure for scattered light intensity:

$$
R_{\theta}=\frac{I_{\text {sample }}-I_{\text {solvent }}}{I_{\text {toluene }}} \times R_{\text {toluene }} \times \frac{n_{\text {solvent }}^{2}}{n_{\text {toluene }}^{2}}
$$


where $I_{\text {sample }}, I_{\text {solvent }}$ and $I_{\text {toluene }}$ are the scattering intensities measured for the sample, the solvent and the toluene reference, $R_{\text {toluene }}$ is the Rayleigh ratio of toluene at the given angle, $n_{\text {solvent }}$ and $n_{\text {toluene }}$ are refractive indexes of the solvent and toluene.

\section{Atomic Force Microscopy}

All AFM samples are prepared from solutions obtained during the DLS titration. We coat clean and hydrophilic silicon wafers with the $\mathrm{Ni}^{2+}$ :(tpy $)_{2} \mathrm{PEO}$ mixtures and leave these for 20 minutes for equilibration. Next the excess of solution is removed and the sample surfaces are washed three times with miliQ for removing salts and non-adsorbed material. Wafers prepared this way are then dried under $\mathrm{N}_{2}$ stream. Sample analysis is performed with a Bruker Nanoscope $\mathrm{V}$ in contact mode using standard $2 \mathrm{~nm}$ radius Bruker SCANASYST-AIR tips. The peak force is used as a feedback mechanism. Images are processed with NanoScope Analysis 1.20 (Veeco Instruments Inc. 2010, USA)

\section{Cryo-SEM}

For the cryo-SEM experiments, we use highly concentrated 20wt\% nickel based gels in a critical gel state. The gel is cut into thin slices with a razor blade. Next we mount the slices into the sample holders coated with carbon adhesive tape. These holders are placed in liquid nitrogen and transferred into the Leica cryoMED020 cryopreparation unit equipped with a high pressure freeze-dryer. When the water is removed from the surface we sputter-coat it with $5 \mathrm{~nm}$ layer of iridium that prevents the sample from charging. The samples are then transferred into the FEI Magellan 400 ultra-high field emission scanning electron microscope (FESEM). Images are recorded digitally.

\subsection{Results and discussion}

Our experimental system consists of flexible water-soluble polyethylene oxide (PEO) chains of $10 \mathrm{kDa}$, whose chain ends have been modified with metalbinding terpyridine (tpy) groups; the synthesis of these telechelic polymers is 
described elsewhere ${ }^{[12,13]}$. The tpy end-groups form tridentate coordination complexes with bivalent transition metal ions $\left(\mathrm{M}^{2+}\right)$; dissolved in apolar media this would only lead to chain extension of the $\mathrm{PEO}(\mathrm{tpy})_{2}$ telechelics due to the 1:2 $M^{2+}$ : tpy binding stoichiometry. However, as we dissolve these polymers in water, hydrophobic interactions between the conjugated coordination complexes provide an additional association mechanism, resulting in the formation of transient networks, as illustrated in Fig.3.1. The typical lifetime $\tau$ of a $\mathrm{M}^{2+}-(\text { tpy })_{2}$ coordination complex depends strongly on the metal ion. For example, iron(II) and nickel(II) form very strong complexes with terpyridine, which dissociate very slowly, with $\tau \simeq 10^{7} \mathrm{~s}$ for $\mathrm{Fe}^{2+}$ and $\tau \simeq 10^{6}$ for $\mathrm{Ni}^{2+}$.
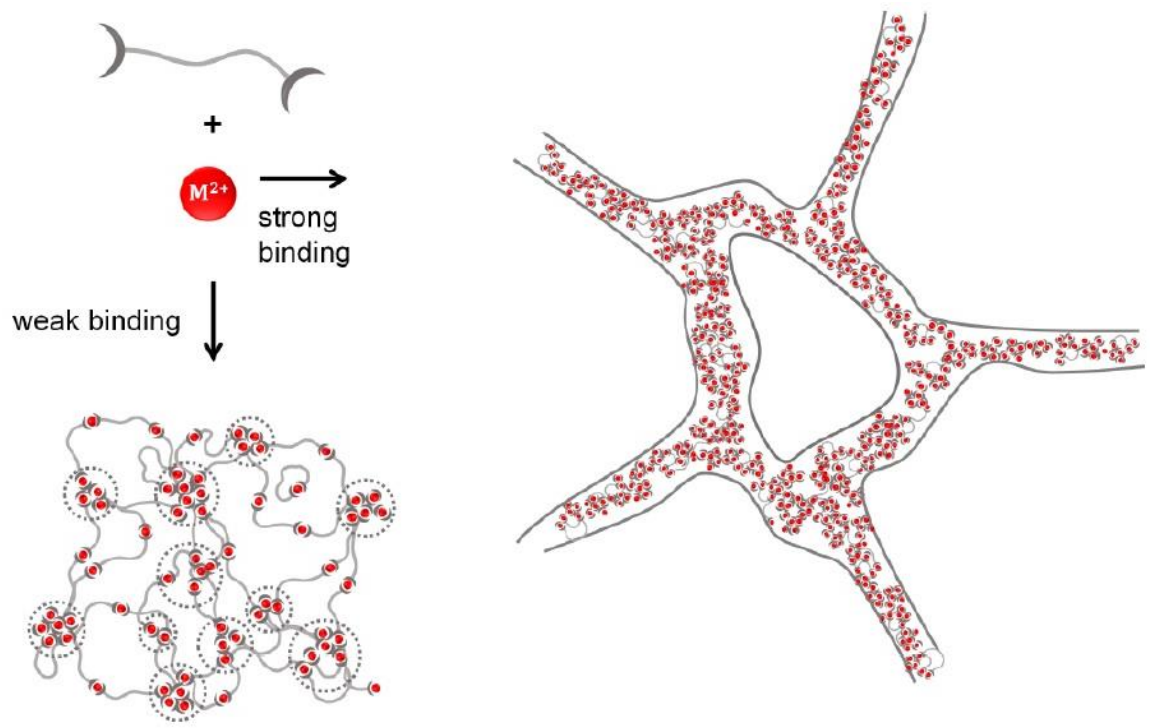

Figure 3.1. Schematic illustration of terpyridine-ligand end-functional polymers whose state can be controlled by the addition of divalent transition metal ions, leading to homogeneous network formation for weakly binding ions, e.g. $\mathrm{Zn}^{2+}$ or $\mathrm{Co}^{2+}$, or phase separation for strongly binding ions such as $\mathrm{Fe}^{2+}$ of $\mathrm{Ni}^{2+}$.

By contrast, cobalt(II) forms complexes of intermediate strength and dissociation rate $\tau \simeq 10^{3} \mathrm{~s}$; whereas zinc(II) forms weak and labile complexes $\tau<1 \mathrm{~s}{ }^{[14,15]}$. This large deference in binding strength allows us to tune the 
dissociation kinetics of the bonds in the network in a very wide range, simply by changing the metal ion. We characterize networks formed at relatively high polymer concentrations of $20 \% \mathrm{w} / \mathrm{v}$ using oscillatory rheology in the linear regime in a cone-plate geometry. In the absence of metal ions no appreciable modulus develops, indicating that entanglements between the PEO chains do not contribute to the rheological properties we explore.

When we add weakly-binding ions, such as $\mathrm{Zn}^{2+}$ or $\mathrm{Co}^{2+}$, whose dissociation kinetics are relatively fast, the frequency dependence of shear elastic $\left(G^{\prime}\right)$ and viscous $\left(G^{\prime \prime}\right)$ moduli exhibits the expected Maxwellian behavior (Fig.3.2a). By contrast, for strongly binding transition metals, such as $\mathrm{Ni}^{2+}$ or $\mathrm{Fe}^{2+}$, we observe distinct critical gel behavior ${ }^{[10,11]}$, where the storage and loss moduli show power-law scaling with angular frequency $G^{\prime} \propto G^{\prime \prime} \propto \omega^{n}$. For both nickel(II) and iron(II) we find a critical exponent of $n=0.3$. The increase in association strength is also apparent when we compare the scale of the shear elasticity, which increases with increasing bind ing constant of the ions: $\mathrm{Zn}^{2+}<\mathrm{Co}^{2+}<\mathrm{Ni}^{2+}<\mathrm{Fe}^{2+}$. The critical behavior of the system is also manifested in the relaxation modulus $G(t)$, which expresses the normalized temporal evolution of shear stress upon application of a step strain of $\gamma=10 \%$ at $t=0$. Also here we find a power-law behavior $G(t) \propto t^{-n}$ with the same characteristic exponent, over almost 4 decades (Fig.3.2b). At times beyond this power-law regime, a terminal relaxation process sets in, leading to an exponential decay of $G(t)$ at long times. This suggests that even in the critical state, for stronglybonded complexes, thermally-activated bond rupture, while slow, still gives rise to mechanical relaxation. These observations give rise to the surprising picture that both types of characteristic response for such telechelic polymer networks, Maxwellian versus critical, may be observed in the same system, merely by changing the association strength of the end groups. This raises the question what microstructural changes occur upon increasing the end group bond strength that leads to this distinct transition in behavior. We hypothesize that this is due to phase separation of a polymer-rich phase from the continuous solvent, which occurs only when the association between end groups is sufficiently strong ${ }^{[16]}$. 




a)

$\omega / \mathrm{rad} / \mathrm{s}$

$10^{2}$



(b)

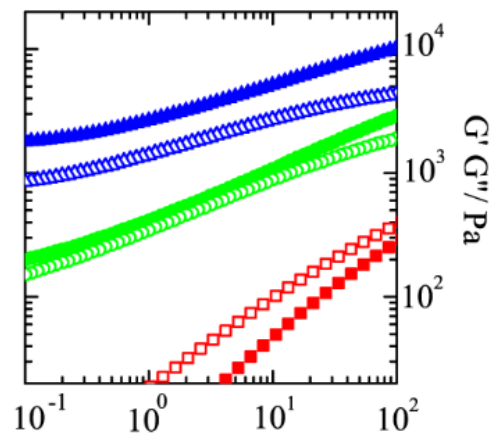

$\omega / \mathrm{rad} / \mathrm{s}$

(c)

Figure 3.2. a) Shear storage $G^{\prime}$ (filled symbols) and loss moduli $G^{\prime \prime}$ (open symbols) as a function of oscillation frequency for telechelic polymer solutions in absence of metal ions (black), with $\mathrm{Zn}^{2+}$ (red), $\mathrm{Co}^{2+}$ (magenta), $\mathrm{Ni}^{2+}$ (green) and $\mathrm{Fe}^{2+}$ (blue); b) Stress relaxation modulus $\mathrm{G}(\mathrm{t})$ upon application of a step strain of $=0: 1$ at $t=0$, for critical gels with $\mathrm{Ni}^{2+}$ (green) and $\mathrm{Fe}^{2+}$ (blue); c) storage filled symbols) and loss (open symbols) moduli as a function of frequency for telechelic polymers with $\mathrm{Ni}^{2+}$ at polymer concentrations of 10,15 and $20 \% \mathrm{w} / \mathrm{v}$.

If such a dense polymer-rich phase begins to demix from the solvent through spinodal decomposition, this may lead the sample to a gel point (Fig.3.1). Once 
the system reaches this gel point in a bicontinuous structure relaxation slows down significantly; in principle this can result in the kinetic arrest of the system exactly at the percolation threshold. This would, in theory, result in critical mechanics, which do not depend on the exact composition of the system. For example, it would be expected to persist for all concentrations sufficiently high to form a percolated structure. Indeed, we find that for very low polymer concentrations crosslinked with $\mathrm{Ni}^{2+}$ no network formation occurs, while all systems above a certain threshold concentration exhibit critical gel behavior (Fig.3.2c).

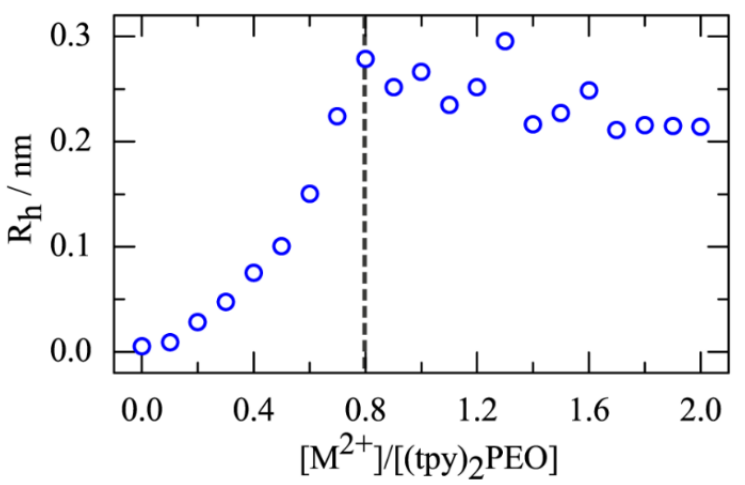

Figure 3.3. Hydrodynamic radius as a function of the molar ratio of $\mathrm{Ni}^{2+}$ to telechelic polymer (tpy) $)_{2} P E O$, at a polymer concentration of $1 \% \mathrm{w} / v$.

A similar scenario is found for the formation of colloidal gels from weaklyattractive microscopic particles; here a glass transition within the dense spinodal phase causes a non-equilibrium state of kinetic arrest resulting in heterogeneous solid-like gel structure ${ }^{[17]}$. Also for colloidal gels, some specific systems exhibit critical gel behavior ${ }^{[18]}$. To verify that phase separation indeed occurs for polymer solutions in presence of strongly binding ions, we perform dynamic light scattering experiments on dilute solutions of tpy functionalized polymers. Upon titration of a weakly-binding metal ion such as $\mathrm{Zn}^{2+}$ no significant change in the hydrodynamic radius of the scatterers is observed. By contrast, the addition of strongly binding $\mathrm{Ni}^{2+}$ ions leads to the formation of large, micron sized, phase 
separated objects from the initially homogeneous polymer solution. A plateau in hydrodynamic radius $R_{h}$ is reached close to the expected molar binding stoichiometry $\left[\mathrm{M}^{2+}\right] /\left[(\text { tpy })_{2} \mathrm{PEO}\right]=1$ (Fig.3.3).
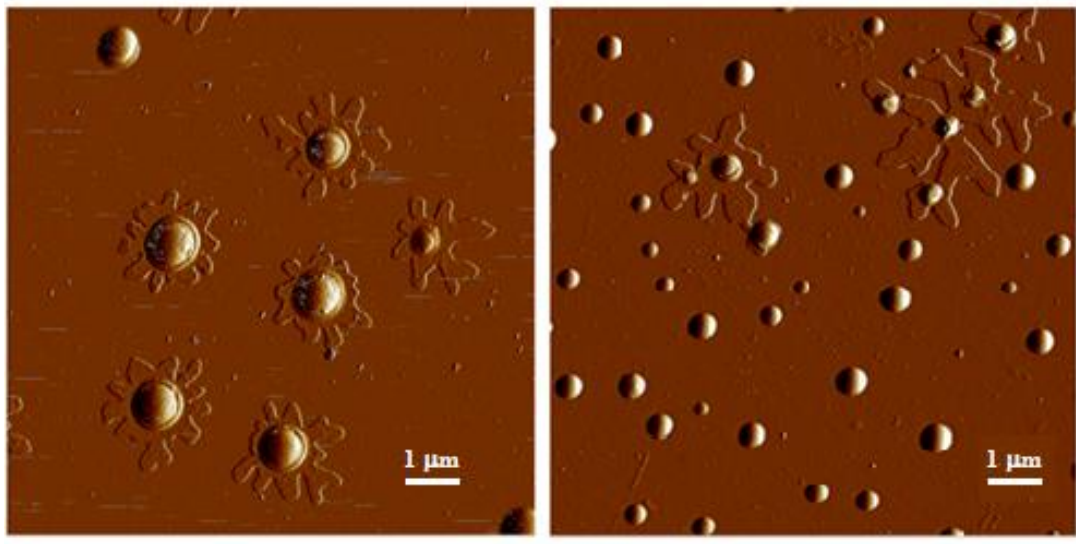

Figure 3.4. AFM peak force image of a titrated solution at $\left[N i^{2+}\right] /\left[(\text { tpy })_{2} \mathrm{PEO}\right]=1$, showing distinct phase separated droplets.

These results are further substantiated by the observation of clear spherical droplets at 1:2 binding stoichiometry using atomic force microscopy (Fig.3.4). The presence of a phase-separated structure in the concentrated polymer solutions which exhibit critical behavior is evaluated using static light scattering experiments. We measure the absolute intensity of scattered light, expressed as the Rayleigh ratio $R_{\theta}(q)$, as a function of the scattering vector magnitude $q$. Indeed, we observe a strong difference between polymer solutions without metal ions (squares in Fig.3.5a) and those in which $\mathrm{Ni}^{2+}$ ions have been added (circles ibidem). These display a strong upturn at low $q$, which indicates the formation of large-scale heterogeneities (circles in Fig.3.5b). The total scattering from such a heterogeneous network is the sum of an ergodic contribution due to thermal density fluctuations, and an excess contribution $R_{e x}(q)$ which arises from nonergodic spatial inhomogeneities of the structure. 




(a)

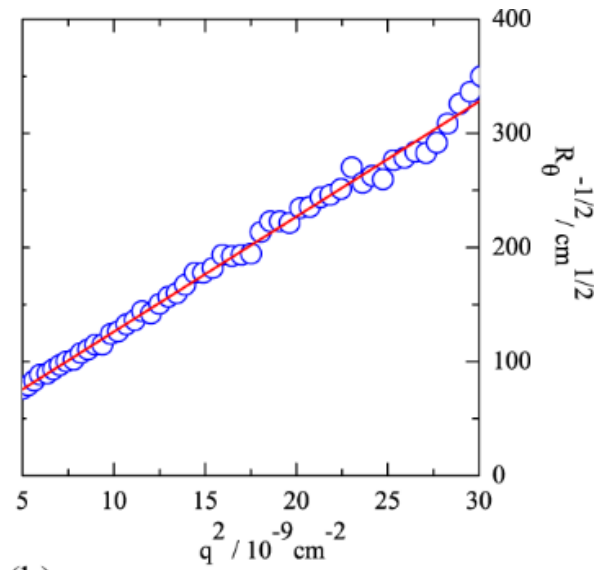

(b)

Figure 3.5. a) Absolute scattering intensity expressed as the Rayleigh ratio $R_{\theta}(q)$ for polymer solutions without metal ions (squares) and with Ni2+ ions at 1:2 $M^{2+}:$ tpy stoichiometry (circles). b) $R_{\theta}^{-1 / 2}$ versus $q^{2}$ for $\mathrm{Ni}^{2+}$-containing gel: experimental data (symbols) and fit to the Debye-Bueche model (drawn line). Inset shows a cryo-SEM image of the spinodal structure inside the same critical gel network.

The $q$ dependence of the latter holds information about the characteristic scale of the heterogeneities. We assume that the ergodic contribution is equal to the total scattering of the pure polymer solution, in which the non-ergodic contribution is absent, to obtain $R_{e x}$ experimentally. The scattering of an inhomogeneous and porous solid is described by the Debye-Bueche equation ${ }^{[19]}$ :

$$
R_{e x}(q)=\frac{4 \pi K \xi^{3}\left\langle\eta^{2}\right\rangle}{\left(1+q^{2} \xi^{2}\right)^{2}}
$$

where $K=8 \pi^{2} n^{2} \lambda^{-4}$, with $n=1.333$ the refractive index of the solvent and $\lambda=633 \mathrm{~nm}$ the wavelength of the incoming laser, $\xi$ the characteristic correlation length of spatial inhomogeneities, and $\left\langle\eta^{2}\right\rangle$ the mean square refractive index fluctuations. 

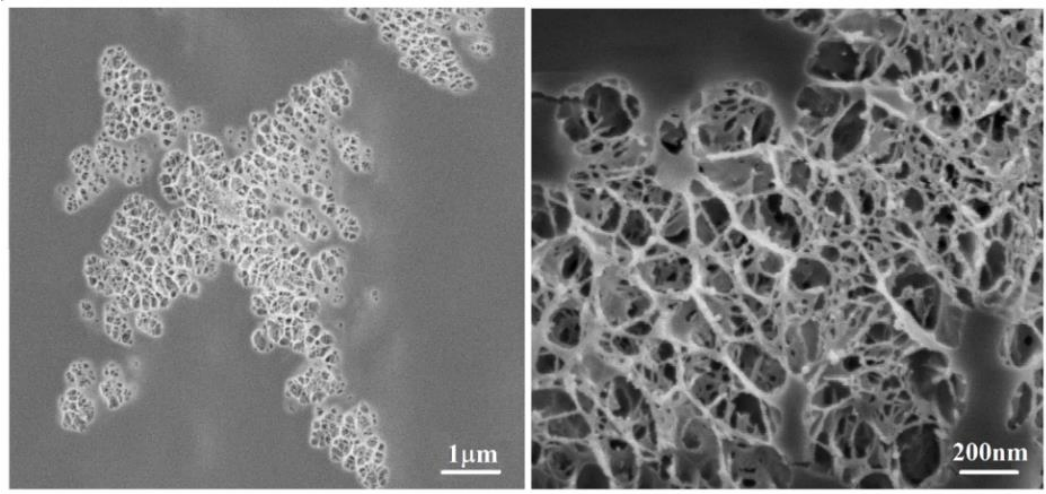

Figure 3.6. Cryo-SEM image of the spinodal structure inside the nickel(II) based critical gel network.

We find excellent agreement between the Debye-Bueche model (solid line in Fig.3.5b) and our experimental data for the critical nickel(II) gel (symbols in Fig.3.5b). From the fit we find a characteristic correlation length of $\xi=169 \pm 8 \mathrm{~nm}$ for the critical $\mathrm{Ni}^{2+}$-crosslinked gels. This is more than an order of magnitude larger than the typical mesh size of $O(5 \mathrm{~nm})$ for the polymer solution in absence of ions, or those crosslinked with weakly-binding ions. The presence of a spinodal structure with a characteristic scale of hundreds of nanometers also becomes apparent from cryoscopic scanning electron microscopy measurements on these gels as shown in Fig.3.6.

The fact that networks which exhibit critical gel-like rheology show phase separation in the dilute regime and a heterogeneous, spinodal structure at high concentrations, supports the hypothesis that critical behavior in these transient networks results from kinetically-arrested phase separation. However, stress relaxation measurements (Fig.3.2b) show that a terminal relaxation time exists for these systems. This implies that the kinetically arrested state cannot persist indefinitely. We do not observe a noticeable evolution for the strongest-binding systems of $\mathrm{Ni}^{2+}$ and $\mathrm{Fe}^{2+}$ over the course of more than a week, probably due to the large bond relaxation time $\left(\tau>10^{6} \mathrm{~s}\right)$. By contrast, networks formed by $\mathrm{Co}^{2+}$ ions, which have a characteristic bond relaxation time of $O\left(10^{3} \mathrm{~s}\right)$, show a distinct temporal evolution in their structure and rheology. The rheology results 
presented in Fig.3.2 were measured on networks which had equilibrated after preparation for several days; clearly these equilibrated $\mathrm{Co}^{2+}$ networks exhibit Maxwellian behavior.

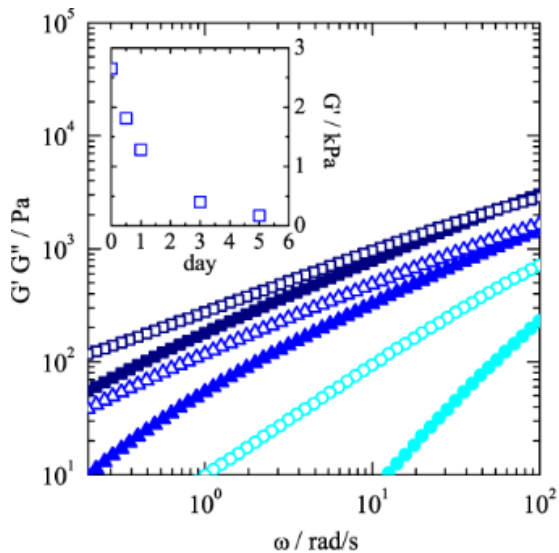

(a)



(b)

Figure 3.7. a) Temporal evolution of the frequency-dependent shear moduli for $20 \% \mathrm{w} / v$ polymer networks crosslinked with $\mathrm{Co}^{2+}$ ions measured at $t=0$ days (blue), 2 days (green) and 5 days (red), inset: shear modulus $G^{\prime}$ at $\omega=100 s^{-1}$ as a function of time after network preparation, b) corresponding evolution of the absolute static scattering intensity $R_{\theta}(q)$ (symbols), with _ts to the DebyeBueche model (drawn lines), inset: correlation length $\xi$ as a function of time.

However, the same networks exhibit critical gel- like behavior directly after preparation; over the course of several days, we observe the relaxation of the system from a rigid material with critical features, to a softer viscoelastic liquid with Maxwellian response (Fig.3.7a). Indeed, the elasticity gradually decays over the course of a week where it tends to a plateau (inset Fig.3.7a). If our speculated explanation of critical gelation by arrested demixing is correct, this should also mean that we would observe the highly heterogeneous structure that forms during phase separation, to slowly dissolve towards the mixed polymer solution. We therefore measure the angle-dependent excess scattering Rex at three different time points during the system evolution and indeed see the 
characteristic length scale $\xi$ decrease as the system relaxes (Fig.3.7b). This is surprising, as a gradual progression of spinodal decomposition would coarsen the system over time. Instead, the system evolves from its temporarily kineticallyarrested state at the gel point towards a homogeneously mixed polymer network with Maxwellian characteristics. Also for colloidal gels, whose bond energies are so large that the frequency of thermally-activated bond rupture is negligibly small, restructuring of the gels has been observed due to internal stresses ${ }^{[20]}$. In this scenario, relaxation leads to coarsening of the structure. The main difference is that the equilibrium state for the polymeric network-formers is a homogeneous network, while the colloidal systems tend to their ground state in the form of a condensed crystalline solid.

\subsection{Conclusions}

Our experiments show how a critical state may emerge in self-assembling polymeric systems due to arrested phase separation. The pathway to equilibrium depends strongly on the ratio of phase separation kinetics and the inherent time scale of bond rupture, which enables mechanical relaxation. For systems that relax slowly but at finite time scales, the critical state is transient and vanishes as thermal rearrangements homogenize the phase separated structure and steer the system towards its equilibrium, Maxwellian, state. By contrast, for systems where the bond energies are so large that spontaneous bond dissociation is halted, the critical state may be long-lived. This highlights how the apparently different mechanical properties of various soft materials with a network structure, may form part of the same continuum. Moreover, it paints an unusual picture of the pathway of network formation in self-assembling polymeric systems which may contain persistent yet transient arrested states. 


\subsection{References}

[1] P. J. Skrzeszewska, J. Sprakel, F. A. de Wolf, R. Fokkink, M. A. Cohen Stuart, J. van der Gucht, Macromolecules 2010, 43, 3542-3548.

[2] Y. Séréro, V. Jacobsen, J. F. Berret, R. May, Macromolecules 2000, 33, 1841-1847.

[3] P. J. Skrzeszewska, F. A. de Wolf, M. W. T. Werten, A. P. H. A. Moers, M. A. Cohen Stuart, J. van der Gucht, Soft Matter 2009, 5, 2057-2062.

[4] C. Ligoure, S. Mora, Rheol Acta 2013, 52, 91-114.

[5] L. Ramos, A. Laperrousaz, P. Dieudonné, C. Ligoure, Physical Review Letters 2011, 107, 148302.

[6] F. Tanaka, S. F. Edwards, Macromolecules 1992, 25, 1516-1523.

[7] J.-F. Le Meins, J.-F. Tassin, Colloid and Polymer Science, 281, 283287.

[8] S. Liu, L. Li, ACS Applied Materials \& Interfaces 2015, 7, 2688-2697.

[9] T. Vermonden, N. A. M. Besseling, M. J. van Steenbergen, W. E. Hennink, Langmuir 2006, 22, 10180-10184.

[10] M. Mours, H. H. Winter, Macromolecules 1996, 29, 7221-7229.

[11] H. H. Winter, F. Chambon, Journal of Rheology 1986, 30, 367-382.

[12] M. Gerth, M. Bohdan, R. Fokkink, I. Voets, J. van der Gucht, J. Sprakel, Macromolecular Rapid Communications 2014, 35, 2065-2070.

[13] B. G. G. Lohmeijer, U. S. Schubert, Macromolecular Chemistry and Physics 2003, 204, 1072-1078.

[14] R. H. Holyer, C. D. Hubbard, S. F. A. Kettle, R. G. Wilkins, Inorganic Chemistry 1966, 5, 622-625.

[15] R. Wang, M. Geven, P. J. Dijkstra, P. Martens, M. Karperien, Soft Matter 2014, 10, 7328-7336.

[16] J. Sprakel, N. A. M. Besseling, M. A. Cohen Stuart, F. A. M. Leermakers, The European Physical Journal E 2008, 25, 163-173.

[17] P. J. Lu, E. Zaccarelli, F. Ciulla, A. B. Schofield, F. Sciortino, D. A. Weitz, Nature 2008, 453, 499-503.

[18] A. S. Negi, C. G. Redmon, S. Ramakrishnan, C. O. Osuji, Journal of Rheology 2014, 58, 1557-1579.

[19] P. Debye, A. M. Bueche, Journal of Applied Physics 1949, 20, 518-525.

[20] T. Hajime, A. Takeaki, EPL (Europhysics Letters) 2007, 79, 58003. 
Critical polymer networks by arrested phase separation 


\section{Linear and nonlinear rheology of metal- coordinated supramolecular networks}

In this chapter we present an extensive rheological study on a supramolecular network formed by terpyridine functionalized water-soluble telechelic polymers. Upon adding nickel ions, the polymers form a transient network, with clusters of metal-ligand complexes as the nodes. We perform rheology in both linear and non-linear response regions, as a function of the ratio between metal and polymer and the overall concentration. At low concentrations, the samples are viscoelastic liquids, while at higher concentrations, a gel is formed. When the ratio between metal and polymer is $1: 1$, which is the optimal ratio for forming $\mathrm{Ni}$ (tpy) $)_{2}$ complexes, the rheology is characterized by a power law scaling, typical for a critical gel. At a slight overdose of metal, this power law behavior disappears, and the response is that of a more traditional viscoelastic material with a characteristic relaxation time. At high deformation rates, the gels show strong shear thinning, with a non-monotonic flow curve that indicates a shear banding instability.

This chapter is a part of the manuscript:

M. Bohdan, J. van der Gucht, J. Sprakel: Critical polymer networks by arrested phase separation. 


\subsection{Introduction}

The rheological behavior of gels formed by telechelic polymers has attracted attention of the scientific community for already several decades. Such polymers are di-functionalized linear chains, where both ends possess a functional group capable of self-assembly ${ }^{[1-6]}$. Often, these functional groups are sticky tails of hydrophobic segments, which are separated by a long hydrophilic chain. In water, these polymers form micelle-like aggregates, in which the middle blocks form a corona of polymeric loops. At higher concentration, loops are converted to bridges that connect different micelles, and eventually the system reaches a percolation threshold, above which a sample-spanning network is formed. Close to the percolation point, telechelic networks, similarly to their chemically cross-linked equivalents, show a characteristic relaxation behavior, in which the complex modulus can be described by a single power law $G^{*}(\omega)=(i \omega)^{\alpha}$ over a wide frequency range ${ }^{[7]}$. The exponent $\alpha$ differs from one material to another and lies usually between 0.1 and $0.8^{[7,8]}$. Gels above the percolation threshold usually do not obey power law scaling, and can often be described with a Maxwell model, consisting of a single relaxation process with a characteristic relaxation time ${ }^{[9]} \tau_{\text {diss }}=\Omega_{0}^{-1} e^{\Delta \mu /\left(k_{B} T\right)}$, where $\Omega_{0}$ is a characteristic frequency of thermal vibration, and $\Delta \mu$ is the potential energy barrier of disengagement. However, it has been reported that some types of telechelic polymers remain kinetically trapped in the critical gel state and do not evolve towards Maxwelllike behavior, even at concentrations far above the percolation threshold ${ }^{[8]}$. The origin of this behavior and the factors that determine what kind of behavior is observed are still poorly understood. In chapter 3 we showed that the rheological signature of a telechelic network is linked strongly to the characteristic time of the functional groups. We did this by investigating a unique hydrogel system consisting of terpyridine functionalized water-soluble telechelic polymers that form networks in the presence of transition metal ions, due to metal-ligand bond formation. We showed that the addition of nickel or iron, which form metal-ligand bonds with long lifetimes, leads to the formation of critical gels, while zinc and cobalt, which form more dynamic metal-ligand bonds, lead to Maxwell-like gels. Terpyridine-functionalized polymers have been 
studied intensively in recent years, among others by the groups of Rowan and Schubert ${ }^{[10-13]}$ and they found application as sensors or self-healing coatings. Also multi-armed backbone designs have been studied, which lead to highly tunable hydrogel networks. Recent findings suggest that the crosslinking in terpyridine modified polymer networks does not only rely on coordination bonds, but also involves the further association of multiple metal-ligand complexes ${ }^{[14-}$ ${ }^{18]}$. In this chapter we will discuss in detail the peculiar rheological behavior of nickel and iron based gels.
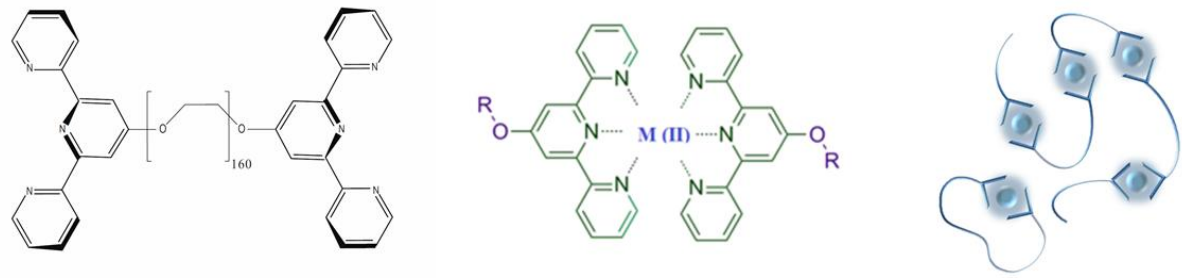

Figure 4.1: Schematic illustration of the assembly by the metal-ligand complexation of terpyridine functionalized telechelic.

First we briefly discuss the gel characterization in the linear response regime, where the concentration and charge dependence will be tested. The remainder of the Chapter then focuses on the investigation of the non-linear response regime where high strains and strain rates are applied. We find strong shearthinning and shear-banding instabilities, in agreement with predictions for telechelic networks by Tanaka and Edwards ${ }^{[9]}$ and Michel et al. ${ }^{[19]}$.

\subsection{Materials and methods}

\section{Chemicals}


chloro- -terpyridine (tpyCl), sodium chloride $(\mathrm{NaCl}, 99.5 \%)$, diethyl ether (99.8\%), 2-(N-morpholino)ethanesulfonic acid (MES) sodium salt, 
$\mathrm{Ni}\left(\mathrm{NO}_{3}\right)_{2} 6 \mathrm{H} 2 \mathrm{O}, \mathrm{FeCl}_{2}, \mathrm{ZnCl}_{2}$ and $\mathrm{CoCl}_{2}$ were purchased from Sigma-Aldrich. Chloroform (HPLC grade) was purchased from Biosolve. Linear poly(ethylene glycol) (PEG) (Mw 10,000g/mol) was purchased from Fluka. All materials were used as received.

\section{Synthesis of $\alpha, \omega$-bis (2,2':6'2"-terpyridin-4'-yl)poly(ethylene glycol)}

The synthesis of $\alpha, \omega$-bis (2,2':6'2"-terpyridin-4'-yl)poly(ethylene glycol) referred further as PEG(tpy) $)_{2}$ is adapted from an existing protocol. A typical synthesis proceeds as follows: Linear PEG (22.4 g, $1.22 \mathrm{mmol})$, KOH powder (0.66 g, $12 \mathrm{mmol}$ ), and $2 \mathrm{~g}$ of molecular sieves $(3 \AA)$ are introduced into a $\mathrm{N}_{2}$-filled twonecked roundbottom, flask. Subsequently, $150 \mathrm{~mL}$ of anhydrous DMSO is added, and the mixture is stirred at $60^{\circ} \mathrm{C}$. After $1.5 \mathrm{hrs}, 1.1 \mathrm{~g}(4.1 \mathrm{mmol})$ tpyCl is added and the reaction is kept at $60^{\circ} \mathrm{C}$ for 64 hrs under constant $\mathrm{N}_{2}$ flow.

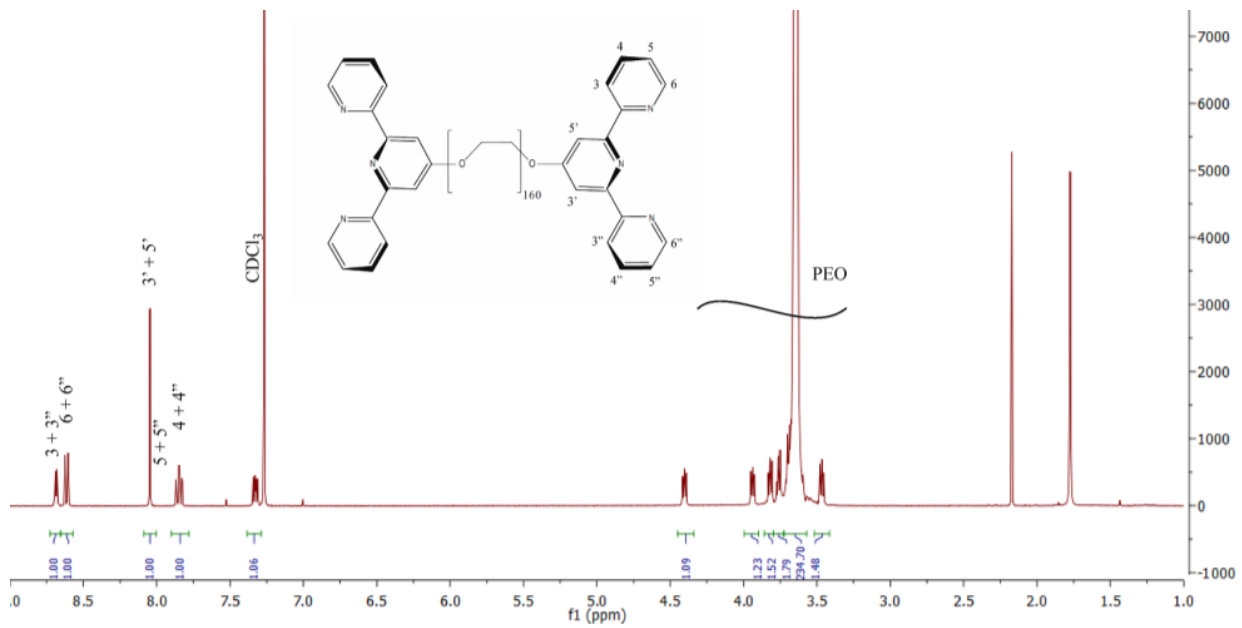

Figure 4.2: ${ }^{1} \mathrm{H}$ NMR spectrum for PEO(tpy) 2

The reaction is stopped by addition of $450 \mathrm{~mL}$ of ice-cold miliQ water, after which the formed precipitate is removed by filtration. The solution is then extracted with chloroform $(4 \times 160 \mathrm{~mL}, 2 \times 80 \mathrm{~mL})$. The organic phase is dried over $\mathrm{Mg}_{2} \mathrm{SO}_{4}$, and concentrated through rotary evaporation (60C, $530 \mathrm{mbar}$ ). 
The product is obtained by precipitation of the concentrated organic phase in 1L of cold diethyl ether. The synthesized polymer was characterized using ${ }^{1} \mathrm{H}-$ NMR and revealed high purity and high conversion into the terpyridine functionalized polymer. Yield: $15.7 \mathrm{~g}$ (68\%). ${ }^{1} \mathrm{H}$ NMR (400MHz, $\mathrm{CDCl}_{3}$, ): 8.69 $(\mathrm{d}, \mathrm{J}=4.5 \mathrm{~Hz}, 4 \mathrm{H}, \mathrm{H} 3, \mathrm{H}$ ), 8.59 (d, J = 7.9Hz, 4H, H6, H ), 7.98 (dt, J = 7.7, $1.8 \mathrm{~Hz}, 4 \mathrm{H}, \mathrm{H} 5, \mathrm{H}$ ), 7.97 (s, 4H, H , H ), 7.47 (ddd, J = 7.7, 4.6, 0.9Hz, 4H, H4, $\mathrm{H}), 4.36(\mathrm{t}, \mathrm{J}=4.9 \mathrm{~Hz}, 4 \mathrm{H}, \mathrm{CH} 2 \mathrm{Otpy}), 3.82$ ( $\mathrm{t}, \mathrm{J}=4.5 \mathrm{~Hz}, 4 \mathrm{H}, \mathrm{CH} 2 \mathrm{CH} 2 \mathrm{Otpy})$, 3.49 ( $\mathrm{s}, \mathrm{CH} 2)$.

\section{Functionalization assay}

The functionalization of the synthesized PEG(tpy $)_{2}$ is assessed by titration of $15 \mu \mathrm{M}$ PEG(tpy) $)_{2}$ solution in 0.1 M MES ( $\mathrm{pH} 5.5$ ) with $\mathrm{Ni}\left(\mathrm{NO}_{3}\right)_{2}$ and analysis of the spectral shifts with UV-VIS spectroscopy. Nickel is used due to the very well-defined absorbance peaks of Ni:(tpy) $)_{2}$ complexes at wavelengths $310 \mathrm{~nm}$ and $320 \mathrm{~nm}$. Upon adding $\mathrm{Ni}\left(\mathrm{NO}_{3}\right)_{2}$ to the polymer solution, we observe an increase in the intensity of both peaks, and a simultaneous quenching of the terpyridine peaks at $280 \mathrm{~nm}$ (Fig. 4.1a).



(a)

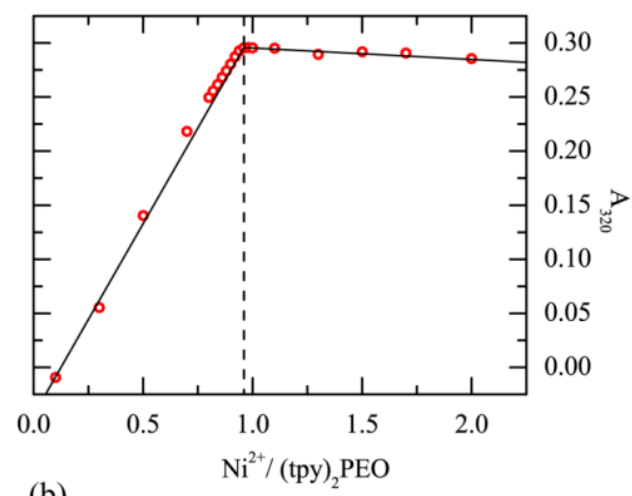

(b)

Figure 4.3: (a) UV-Vis spectra obtained during the titration of PEO(tpy) 2 solution with $\mathrm{Ni}\left(\mathrm{NO}_{3}\right)_{2} ;$ (b) absorbance $320 \mathrm{~nm}$ peak versus nickel ratio.

The intensity of the $320 \mathrm{~nm}$ peak increases linearly with the amount of added metal, and saturates when the ratio between nickel and tpy groups is 1:2. 
From the kink in the curve, we determine the degree of functionalization of the polymer to be $98 \%$ (i.e. on average 1.96 terpyridine groups per polymer).

\section{Sample preparation}

We define the metal-polymer ratio as a $f=\frac{\left[\mathrm{M}^{2+}\right]}{[\mathrm{pol}] n}$, where $n$ is the functionalization degree. Samples with different polymer and metal concentration are prepared from stock solutions of $30 \% \mathrm{PEO}(\mathrm{tpy})_{2}$ and $0.1 \mathrm{M}$ $\mathrm{Ni}\left(\mathrm{NO}_{3}\right)_{2}, \mathrm{FeCl}_{2}, \mathrm{ZnCl}_{2}$ and $\mathrm{CoCl}_{2}$. To improve mixing we add metal solution in a drop-wise manner into an intensively stirred solution of polymer. All samples were mixed vigorously and left for 3 days to equilibrate.

\section{Rheology}

Rheological experiments for all samples are performed with a strain-controlled rheometer (Anton Paar MRC 501), using a cone-plate geometry $(\mathrm{gap}=0.0048 \mathrm{~mm}$, diameter $=50 \mathrm{~mm})$. Temperature is controlled by a Peltier system and it is kept constant at $21^{\circ} \mathrm{C}$. To protect samples from drying, the geometry is covered with silicon oil as a solvent trap. After loading the samples in the rheometer, they are equilibrated for 10 minutes by applying an oscillatory shear with an amplitude of $1 \%$ and a frequency of $1 \mathrm{~Hz}$. The samples consecutively are left to rest for an additional 10 minutes.

Frequency sweep experiments are performed in the linear response regime, at $5 \%$ strain. We follow the evolution of the viscoelastic response with decreasing frequency between 100 and $0.1 \mathrm{rad} / \mathrm{s}$. Flow curves are based on the stress response to a shear rate applied in a range from 0.01 to $100 \mathrm{~s}^{-1}$. Strain sweep measurements are performed in a deformation range from 0.1 to $10000 \%$. In some cases the maximum frequency that could be reached was lower, because the sample was ejected from the measurement cell due to a Weissenberg instability. 


\subsection{Results and discussion}

We have synthesized polymers functionalized with a terpyridine group at each end. These groups are capable of binding metal ions. As we showed in chapter 2 , this leads to the formation of hydrogel networks, in which clusters of metal-ligand complexes form the nodes. Here, we present a detailed characterization of these networks, using nickel as the metal ion.

\subsubsection{Terpyridine binding conditions}

Metal-ligand coordination complexes are very sensitive to the $\mathrm{pH}$. At low $\mathrm{pH}$, the terpyridine groups become protonated, preventing the binding of metal ions. At high $\mathrm{pH}$, hydrolysis of the metal ions leads to strong hydroxide complexes. We therefore performed all our experiments in the presence of $1 \mathrm{M}$ MES buffer of $\mathrm{pH} \mathrm{5,} \mathrm{at} \mathrm{which} \mathrm{both} \mathrm{ligand} \mathrm{protonation} \mathrm{and} \mathrm{metal} \mathrm{hydroxide}$ complex formation are negligible.

\subsubsection{Metal ion ratio and concentration dependence}

In Chapter 3, we observed the formation of critical gels, with power law rheology, for telechelic polymers with nickel and iron ions in a ratio 1:1. We argue that this type of gelation results from the arrested phase separation related directly to the slow dissociation rates of the metal-ligand complexes. Iron and nickel, for example, which have low dissociation rates form very strong and stable complexes whereas zinc and cobalt complexes are much weaker. Therefore, for the same polymer we can observe large differences in binding strength upon varying the type of metal ion, resulting in different types of polymeric networks. Next, we study how the rheological properties depend on the concentration. We do this for two metal/polymer ratios, $f=1$ (Fig. 4.3a and Fig. 4.3b) and $f=1.1$ (Fig. 4.3c and Fig. 4.3d) (note that the data for the $f=1$ ratio were already shown in chapter 3 ). At a concentration of $10 \mathrm{mM}$, the samples are liquid-like at both ratios, with a loss modulus that is higher than the storage modulus at all frequencies. As the concentration increases, both 
moduli increase and the samples become more gel-like. At both ratios the percolation transitions occurs at a concentration of approximately $15 \mathrm{mM}$.
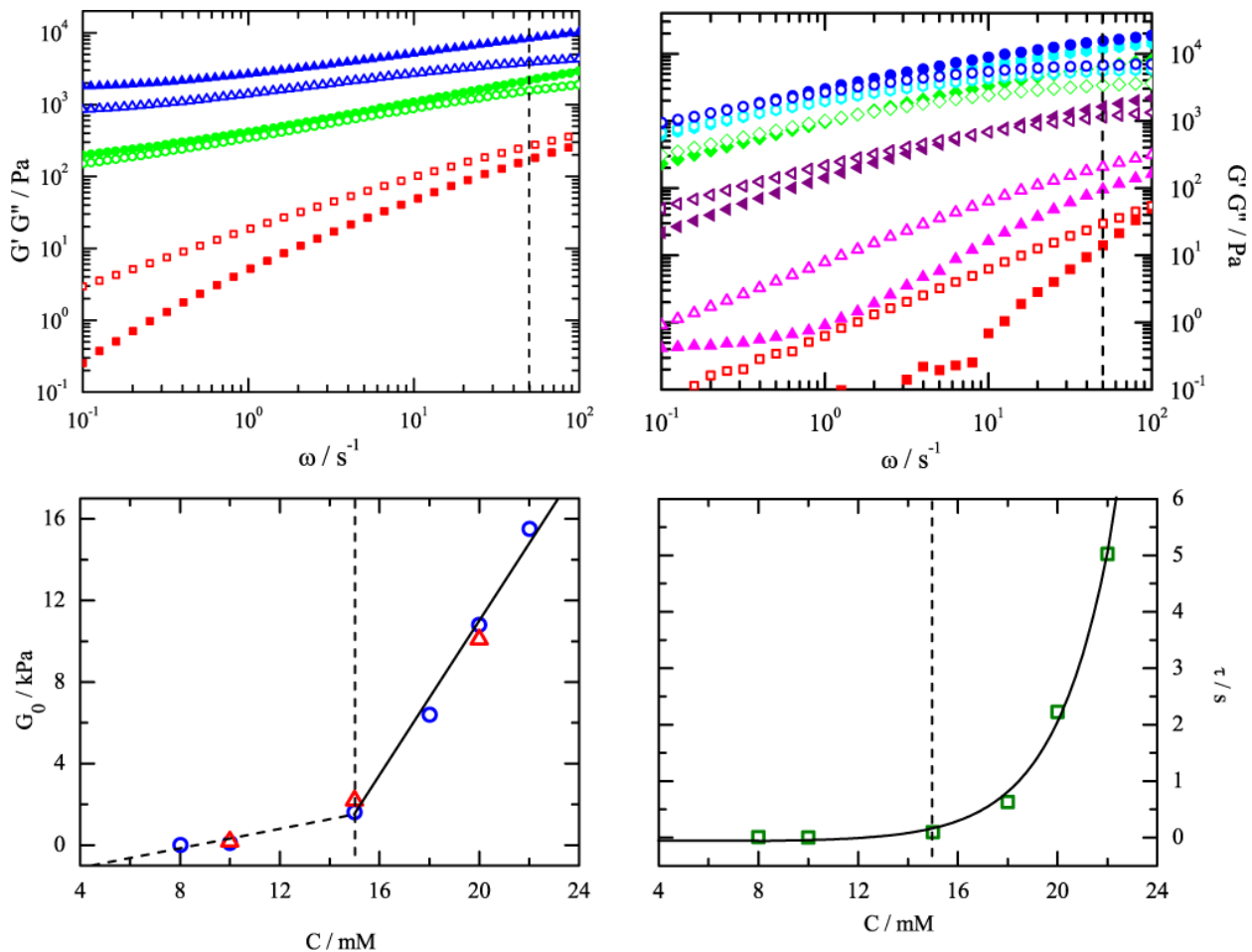

Figure 4.4: Results of the oscillatory experiment for nickel based gels in two metal ion ratios. (a) Frequency sweep for $f=1$ stoichiometry at concentrations of 10 (red squares), 15 (green circles) and 20mM (blue triangles); (b) Plateau modulus $G_{0}$ obtained from the high frequency $\left(50 \mathrm{~s}^{-1}\right)$ storage modulus $G^{\prime}$; (c) frequency sweep for $f=1.1$ stoichiometry over a range of concentrations: 8 $m M$ (red squares), $10 \mathrm{mM}$ (magenta triangles), $15 \mathrm{mM}$ (purple triangles), 18 $m M$ (green diamonds), $20 \mathrm{mM}$ (cyan hexagons) and 22mM (blue circles) open symbols represent loss modulus $G$ "; (d) Plateau modulus $G_{0}$ obtained from the high frequency $\left(50 \mathrm{~s}^{-1}\right)$ storage modulus $G^{\prime}$ (blue circles) and relaxation times of the networks obtained from the crossover between storage and loss moduli (red squares). 
However, upon closer inspection, we see a clear difference in the dynamic response between the samples. At ratio $f=1$, immediately after crossing the gel point the response shows the power law behavior typical for critical gels, where the storage modulus and the loss modulus run parallel over a wide frequency range. Samples with $f=1.1$ are more similar to classical physical gels, with a distinguishable cross-over of the moduli that indicates the time of relaxation of the nodes.

From the crossover of the viscous and elastic moduli, we can estimate the relaxation time for the network nodes (Fig. 4.3d). The relaxation time follows the same trend with concentration as the storage modulus. By crossing the percolation threshold, we see a very pronounced increase of the relaxation time, but even at the highest concentration of $22 \mathrm{mM}$, we are not able to reach the point where the storage modulus dominates the loss modulus over all frequencies.

\subsubsection{Non-linear rheology}

In this paragraph we consider supramolecular networks at large deformations, in the non-linear response regime. For that we subject the transient gels to shear flow at shear rates in a broad range from $10^{-2}$ to $10^{3} s^{-1}$ and we examine the stress response. In figure 4.5 we show results for networks crosslinked by four third row transition metal ions: nickel, iron, zinc, and cobalt, where the increasing binding constant of the ions follows $\mathrm{Zn}^{2+}<\mathrm{Co}^{2+}<\mathrm{Ni}^{2+}<$ $\mathrm{Fe}^{2+}$. For nickel and iron, the stress shows a non-monotonic dependence on the applied shear rate, with a maximum followed by a region of negative slope. By contrast, for zinc and cobalt, the response is that of a Newtonian liquid, with a stress that is proportional to the shear rate at all shear rates. Why the observations for these particular metal ions are so different, was already discussed in Chapter 3, where the rheology in the linear regime response was investigated. We argued there that the performance of each sample depends strongly on the lifetimes of the coordination complexes. Iron (II) and nickel (II) 
form very strong and stable complexes with terpyridine, with long lifetimes, respectively $\tau \simeq 10^{7} \mathrm{~s}$ and $\tau \simeq 10^{6} \mathrm{~s}$. By contrast, cobalt (II) and zinc (II) complexes form much weaker coplexes, with lifetimes $\tau \simeq 10^{3} s$ and $\tau<1 s$, respectively ${ }^{[20-22]}$.

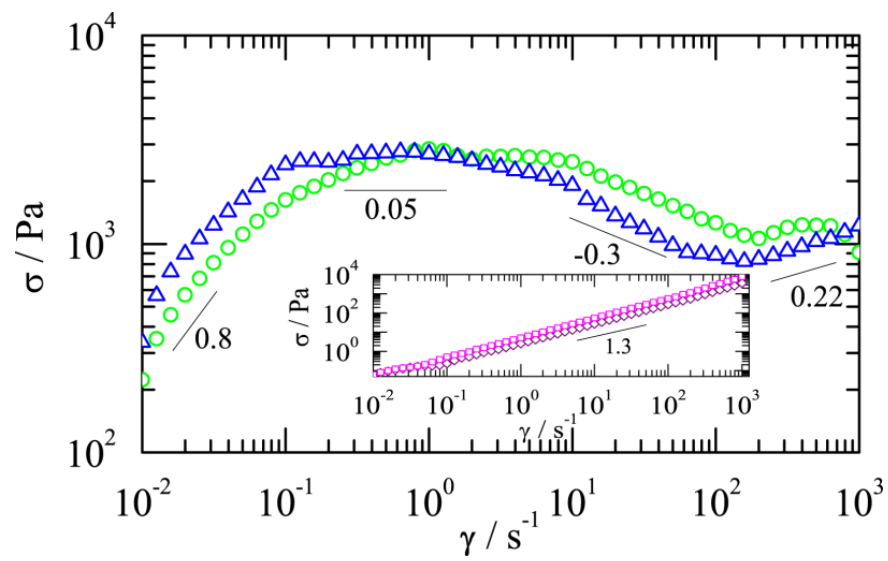

Figure 4.5: Flow curves for nickel (green circles) and iron (blue triangles) based gels. Inset shows Newtonian flow for equilibrated samples with cobalt (magenta squares) and zinc (purple diamonds)

The flow curves of iron and nickel based gels show similar behavior. We can distinguish a few specific regions. Already at very low shear rates, $\dot{\gamma}<0.1$, the networks are shear thinning (with $\partial \ln \sigma / \partial \ln \dot{\gamma}=0.8$.) and above shear rates of $0.1 s^{-1}$, the slope decreases even further and eventually even becomes negative. This region of negative slope is an indication of a flow instability, leading to a non-homogeneous flow profile, called shear-banding. Similar flow instabilities have been observed previously for wormlike micelles, colloidal suspensions and entangled polymer solutions ${ }^{[23-25]}$. The origin of shear banding is a nonmonotonic constitutive relation due to extreme shear thinning. It is easily shown that the region in which the stress decreases with increasing shear rate is mechanically unstable ${ }^{[25]}$. When the applied overall shear rate lies within this unstable region, the fluid separates into two regions of different shear rate. 
The establishment of this non-homogeneous flow profile can take a long time ${ }^{[24]}$, so that the non-monotonic stress-shear rate relation can be observed as a metastable state.

An explanation for the non-monotonic underlying constitutive relation of transient networks has been given by Tanaka and Edwards and later also by Sprakel et al, based on the force-activated disruption of the physical crosslinks in the network ${ }^{[9,24]}$. Under shear flow, the polymer strands in the network are stretched, leading to an elastic force pulling on the crosslinks. This force helps to overcome the energy barrier for bond dissociation, and thereby enhances the dissociation rate. At low shear rates, the reformation of crosslinks is sufficiently fast to restore the amount of crosslinks, so that the viscosity is hardly affected by the flow. However, at high shear rates the effective number of crosslinks is strongly reduced, leading to a strong, almost catastrophic decrease of the viscosity. This produces the region of negative slope in the flow curve. At very high shear rates, the stress increases again, for example because the solvent viscosity starts to play a role.

To study the instability of the gels in more detail, we perform strain sweep experiments. First, we consider two samples at three concentrations at $f=1$, in the critical gel state.

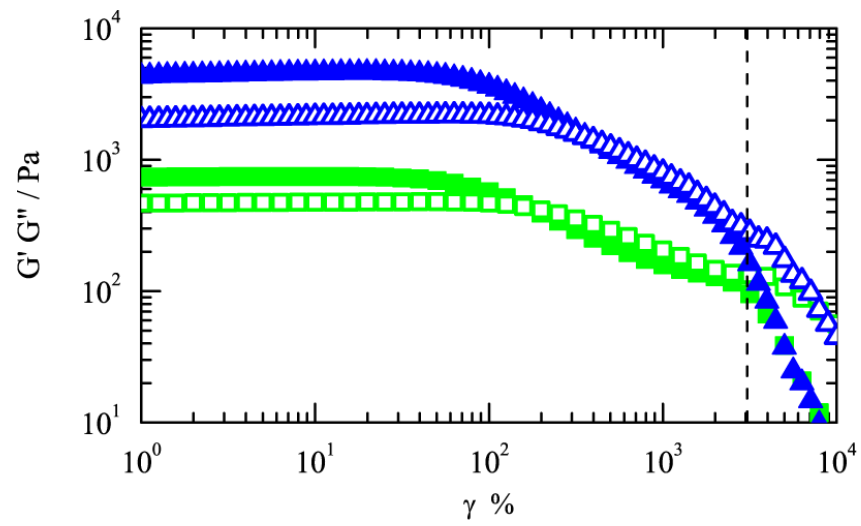

Figure 4.6: Strain sweep of sample at concentration of 20mM (blue symbols) and $15 \mathrm{mM}$ (green symbols, at the metal ion - functionality degree balance $f=$ 1. Open symbols stand for loss modulus and full samples for storage modulus. 
The gel is deformed in a range from $1 \%$ to 10 000\% with the frequency fixed at $1 \mathrm{rad} / \mathrm{s}$. As shown in Figure 4.6, for both samples we observe a decrease of the moduli above a certain strain amplitude, indicating yielding of the gels. We read the critical strain from the crossover of the storage and loss moduli associated with the samples yielding. This depends on the sample concentration, and is approximately $350 \%$ for the $20 \mathrm{mM}$ sample and $150 \%$ for the $15 \mathrm{mM}$ sample.

In both cases, we observe a gradual decrease of both storage and loss moduli after the yield point, probably related to a non-homogeneous deformation profile. Around 3000\%, the flow of both samples becomes macroscopically unstable. This instability results in the expulsion of the sample from the geometry and is known as edge fracture or the Weissenberg effect. Typically, the Weissenberg effect occurs for viscoelastic systems with relatively long relaxation times. It is a result of normal stresses that arise in a viscoelastic liquid under shear. When the normal stress reaches a critical value, a crack appears at the free liquid/air surface, leading to an instability that finally expels the liquid out of the gap.

We repeat the experiment for the sample at $20 \mathrm{mM}$, for different applied frequencies (figure 4.7a). We obtain the yield point at each frequency from the crossover of the storage and loss moduli. As shown in figure $4.7 \mathrm{~b}$, the critical strain at which the sample yields increases logarithmically with the applied deformation frequency. This logarithmic dependence can be explained using a model which is based on the activated bond rupture theory of Kramers ${ }^{[23,26]}$. According to this theory, the dissociation rate of a bond increases exponentially with the force acting on it, $k_{D}^{\prime}=k_{D} \cdot \exp [f \delta / k T]$, where $k_{D}$ is the dissociation rate of the crosslink, which is the inverse of the lifetime of the bond $\tau$, and where $\delta$ is a characteristic activation length. In our experiments, the force acting on the crosslinks is not constant, but increases as the polymer chains are stretched by the applied shear flow. To describe the bond rupture kinetics we follow the approach of Skrzeszewska et al, which is based on the single bond model of Evans [27] and the adhesive rupture model of Chaudhury ${ }^{[28]}$. By assuming that the deformation is applied homogeneously, each chain in the network is stretched at a rate $\xi \dot{\gamma}$, where $\xi$ is the average mesh size. 
Treating each polymer as a Gaussian chain with spring constant $k T / \xi^{2}$, the force applied to the node can be written as $f(t)=K(k T / \xi) \dot{\gamma t}$, where $\mathrm{K}$ stands for local stress intensification due to inhomogeneities in the sample. By substituting this transient force into the Kramers relation for the dissociation rate, we see that $k_{D}^{\prime}$ is increasing exponentially with time.

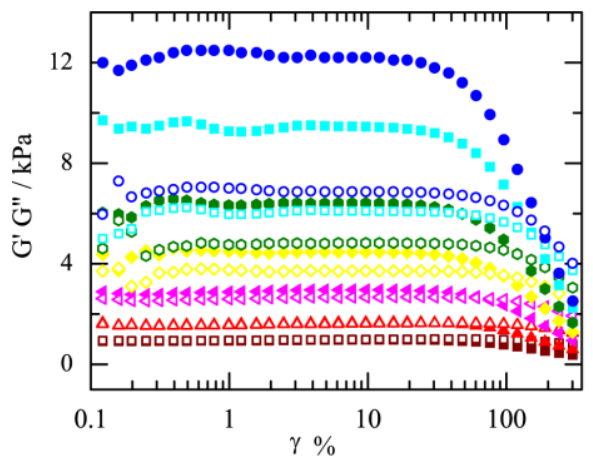

(a)



(b)

Figure 4.7: (a) Strain sweep of sample at fixed concentration $20 \mathrm{mM}$ over a range of different oscillation frequencies. Full symbols represent storage modulus $G$ ' and open symbols represent loss modulus $G$ "; (b) yield strain of $20 \mathrm{mM}$ gel as a function of oscillation frequency in strain sweep experiment.

Therefore, we can write the survival probability of the node, $P(t)$ as:

$$
P(t)=\exp \left\{-\int_{0}^{t} k_{D}^{\prime}[f(t)] d t\right\}=\exp \left\{\frac{\xi}{K \delta \dot{\gamma} \tau}\left[1-\exp \left(\frac{K \delta \dot{\gamma} \tau}{\xi}\right)\right]\right\}
$$

with $\tau=1 / k_{D}$. From this we obtain the average lifetime of a node as:

$$
\left\langle\tau_{0}^{\prime}\right\rangle=\int_{0}^{\infty} P(t) d t \approx \begin{cases}\tau\left(1-\frac{K \delta \dot{\gamma} \tau}{\xi}\right) & \text { if } \dot{\gamma} \tau \ll \frac{\xi}{k \delta} \\ \frac{\xi}{K \delta \dot{\gamma}} \ln \left(\frac{K \delta \dot{\gamma} \tau}{\xi}\right) & \text { if } \dot{\gamma} \tau \gg \frac{\xi}{k \delta}\end{cases}
$$

For high shear rates, crosslink recombination can be neglected and the network experiences a catastrophic failure after a time $t_{b}^{\prime} \approx\left\langle\tau_{0}^{\prime}\right\rangle$. From this we can write the critical strain for rupture as: 


$$
\gamma_{c}=\dot{\gamma} t_{b}^{\prime} \approx \frac{\xi}{K \delta} \ln \left[\frac{K \delta \dot{\gamma} \tau}{\xi}\right]
$$

Hence, the model predicts that the critical strain increases logarithmically with the applied shear rate in agreement with the experimental results shown in Figure $4.7 \mathrm{~b}$. From this result we can estimate how homogeneously the stress is distributed in the sheared sample. We do this by fitting equation 4.3 to the experimental data. From the value of the slope, and using $\xi=160 \mathrm{~nm}$ and $\delta=0.5 \mathrm{~nm}$ we estimate the local stress intensification factor $K=9.33$. This means that the stress in the region where yielding starts is about 9 times higher than the average stress.

\subsection{Conclusions}

In this chapter we have presented an extensive rheological study of a supramolecular network formed by terpyridine functionalized water-soluble telechelic polymers. We found out that the critical gel state described in Chapter 3 occurs only at fixed stoichiometry where the concentration of added metal ion is equal to the concentration of the polymers, giving a $1: 2 \mathrm{M}^{2+}$ tpy binding stoichiometry, which is optimal for chain extension. When either metal or polymer is in excess, the rheological behavior shows a more traditional signature of a transient gel, with a dominant characteristic relaxation time. Concentration-dependent measurements, at two different metal/polymer ratios allowed us to observe the percolation threshold for gel formation and to assess the character of the sol-critical gel transition. In the non-linear regime, the samples showed strong shear thinning, indicative of a shear-banding instability, with the associated large stress fluctuations and metastable states and the socalled Weissenberg effect at higher shear rates, where the sample is expelled from the gap due to the large normal force. We explain this behavior using a model based on the assumption of stress-activated crosslink rupture. 


\subsection{References}

[1] T. Annable, R. Buscall, R. Ettelaie, D. Whittlestone, Journal of Rheology 1993, 37, 695-726.

[2] C. Charbonneau, C. Chassenieux, O. Colombani, T. Nicolai, Physical Review E 2013, 8\%.

[3] M. Gerth, M. Bohdan, R. Fokkink, I. Voets, J. van der Gucht, J. Sprakel, Macromolecular Rapid Communications 2014, 35, 2065-2070.

[4] F. Laflèche, D. Durand, T. Nicolai, Macromolecules 2003, 36, 13311340.

[5] M. Lemmers, J. Sprakel, I. K. Voets, J. van der Gucht, M. A. Cohen Stuart, Angewandte Chemie International Edition 2010, 49, 708-711.

[6] T. Vermonden, J. van der Gucht, P. de Waard, A. T. M. Marcelis, N. A. M. Besseling, E. J. R. Sudhölter, G. J. Fleer, M. A. Cohen Stuart, Macromolecules 2003, 36, 7035-7044.

[7] H. Winter, M. Mours, in Neutron Spin Echo Spectroscopy Viscoelasticity Rheology, Vol. 134, Springer Berlin Heidelberg, 1997, pp. 165-234.

[8] T. Vermonden, N. A. M. Besseling, M. J. van Steenbergen, W. E. Hennink, Langmuir 2006, 22, 10180-10184.

[9] F. Tanaka, S. F. Edwards, Macromolecules 1992, 25, 1516-1523.

[10] S. Bode, L. Zedler, F. H. Schacher, B. Dietzek, M. Schmitt, J. Popp, M. D. Hager, U. S. Schubert, Adv. Mater. 2013, 25, 1634-1638.

[11] H. Hofmeier, U. S. Schubert, Chemical Society Reviews 2004, 33, 373399.

[12] P. K. Iyer, J. B. Beck, C. Weder, S. J. Rowan, Chemical Communications 2005, 319-321.

[13] A. Winter, G. R. Newkome, U. S. Schubert, ChemCatChem 2011, 3 , 1384-1406.

[14] T. Rossow, A. Habicht, S. Seiffert, Macromolecules 2014, 47, 64736482 .

[15] T. Rossow, S. Seiffert, Polymer Chemistry 2014, 5, 3018-3029.

[16] W. Weng, J. B. Beck, A. M. Jamieson, S. J. Rowan, Journal of the American Chemical Society 2006, 128, 11663-11672.

[17] W. Weng, Z. Li, A. M. Jamieson, S. J. Rowan, Soft Matter 2009, 5, 4647-4657. 
[18] W. Weng, Z. Li, A. M. Jamieson, S. J. Rowan, Macromolecules 2009, 42, 236-246.

[19] E. Michel, J. Appell, F. Molino, J. Kieffer, G. Porte, Journal of Rheology 2001, 45, 1465-1477.

[20] I. M. Henderson, R. C. Hayward, Polymer Chemistry 2012, 3, 12211230.

[21] R. H. Holyer, C. D. Hubbard, S. F. A. Kettle, R. G. Wilkins, Inorganic Chemistry 1966, 5, 622-625.

[22] R. Wang, M. Geven, P. J. Dijkstra, P. Martens, M. Karperien, Soft Matter 2014, 10, 7328-7336.

[23] P. J. Skrzeszewska, J. Sprakel, F. A. de Wolf, R. Fokkink, M. A. Cohen Stuart, J. van der Gucht, Macromolecules 2010, 43, 3542-3548.

[24] J. Sprakel, E. Spruijt, M. A. Cohen Stuart, N. A. M. Besseling, M. P. Lettinga, J. van der Gucht, Soft Matter 2008, 4, 1696-1705.

[25] J. van der Gucht, M. Lemmers, W. Knoben, N. A. M. Besseling, M. P. Lettinga, Physical Review Letters 2006, 97, 108301.

[26] H. A. Kramers, Physica 1940, 7, 284-304.

[27] E. Evans, K. Ritchie, Biophysical Journal 1997, 72, 1541-1555.

[28] M. K. Chaudhury, The Journal of Physical Chemistry B 1999, 103, 6562-6566. 


\section{Supramolecular Assembly of Self-Healing Nanocomposite Hydrogels}

Hierarchical self-assembly of transient composite hydrogels is demonstrated through a two-step, orthogonal strategy using nanoparticle tectons interconnected through metal-ligand coordination complexes. The resulting materials are highly tunable with moduli and viscosities spanning many orders of magnitude, and show promising self-healing properties, while maintaining complete optical transparency.

This chapter is a part of the manuscript:

M. Gerth, M. Bohdan, R. Fokking, I. Voets J. van der Gucht, J. Sprakel: Supramolecular Assembly of Self-Healing Nanocomposite Hydrogels 


\section{$5.1 \quad$ Introduction}

Physical gels are polymeric networks formed from building blocks linked together through non-covalent interactions. The transient nature of the bonds that provide mechanical stability to these networks enables the resulting materials to reshape their microstructure and hence dynamically adapt to external stresses and stimuli. These adaptive soft solids often exhibit enhanced stability against catastrophic failure, as compared to their covalent counterparts, and can regenerate their structure $[1,2]$.

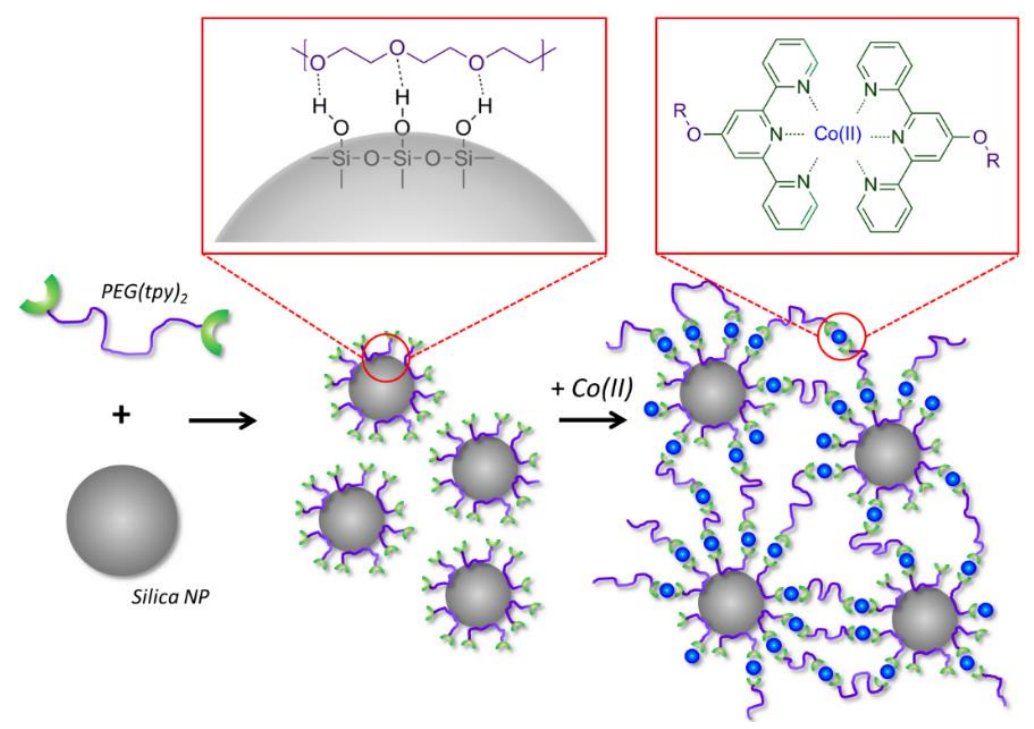

Figure 5.1. Schematic representation of network formation. Multifunctional tectons are formed by adsorption of bifunctional PEG onto silica nanoparticles, which are subsequently linked by Co(II)-terpyridine coordination.

Moreover, the strength and kinetics of the physical bonds can be regulated using external triggers; this allows facile, non-chemical, routes to tailor the structure and mechanics [3, 4]. This approach has been extensively explored to create gels from small molecular or polymeric building blocks interacting through for example hydrogen bonding [5], metal-coordination [4], electrostatic attraction 
$[6,7]$, or combinations of metal-ligand and hydrophobic interactions [8]. Noncovalent interactions can also arise between polymer chains and solid surfaces; introducing nanoparticles in covalent polymer gels leads to increasing elasticity and fracture energy through physical adsorption of polymers onto nanoparticle surfaces [9].

However, introducing nanoparticles into polymer solutions [10], melts [11] or networks [9], in many cases leads to flocculation of the particles, resulting in highly heterogeneous and opaque networks. In this chapter, we present a strategy for the bottom-up assembly of transparent and homogeneous composite hydrogels formed exclusively through noncovalent bonding. First, we create colloidal building blocks through spontaneous adsorption of terpyridine endcapped polymers onto silica nanoparticles. Subsequently, these multi-valent tectons are assembled into composite hydrogels by addition of $\mathrm{Co}$ (II), creating transient metal-coordination linkages between the tectons (see Fig.5.1. ). We choose $\mathrm{Co}$ (II) as the metal ion, because it is known to form strong, yet reversible coordination complexes with terpyridine groups in aqueous environment [12]. We show that the mechanics of the resulting networks can be tuned over many orders of magnitude and that, upon breaking, these networks spontaneously restore their mechanical properties within several minutes.

\subsection{Materials and Methods}

\section{Materials}

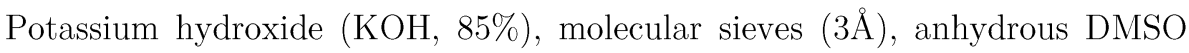
(99.9\%), 4'-chloro-2,2':6'2"-terpyridine (tpyCl), $\mathrm{NaCl}$ (99.5\%), diethyl ether (99.8\%), 2-( $N$-morpholino)ethanesulfonic acid (MES) sodium salt, DMSO- $d_{6}$ and Ludox AM were purchased from Sigma-Aldrich. Chloroform (HPLC grade) was purchased from Biosolve. Linear poly(ethylene glycol) (PEG) (Mw 10,000g/mol) was purchased from Fluka. $\mathrm{CoCl}_{2} \cdot 6 \mathrm{H}_{2} \mathrm{O}$ was purchased from J.T. Baker. All materials were used as received. 


\section{Synthesis of $\alpha, \omega$-bis(2,2':6'2'-terpyridin-4'-yl)poly(ethylene glycol)}

The synthesis of PEG(tpy) $)_{2}$ is adapted from an existing protocol[13]. Linear PEG (12.18 g, $1.22 \mathrm{mmol})$, KOH powder (0.66 g, $11.76 \mathrm{mmol})$, and $2 \mathrm{~g}$ of molecular sieves $(3 \AA)$ are introduced into a $\mathrm{N}_{2}$-filled two-necked round-bottom flask. Subsequently, $150 \mathrm{~mL}$ of anhydrous DMSO is added, and the mixture is stirred at $60^{\circ} \mathrm{C}$. After $1.5 \mathrm{hrs}, 1.09 \mathrm{~g}(4.07 \mathrm{mmol})$ tpyCl is added and the reaction is kept at $60^{\circ} \mathrm{C}$ for $64 \mathrm{hrs}$ under constant $\mathrm{N}_{2}$ flow. The reaction is stopped by addition of $450 \mathrm{~mL}$ of cold miliQ water, after which the formed precipitate is removed by filtration. The solution is then extracted with chloroform $(4 \times 160$ $\mathrm{mL}, 2 \times 80 \mathrm{~mL}$ ). The organic phase is dried over $\mathrm{Na}_{2} \mathrm{SO}_{4}$, and concentrated through rotary evaporation $\left(60^{\circ} \mathrm{C}, 530 \mathrm{mbar}\right)$. The product is obtained by precipitation of the concentrated organic phase in $1 \mathrm{~L}$ of cold diethyl ether. The precipitate is removed through filtration and washed with cold diethyl ether. The precipitate is dried using a Schlenk line with a cold trap. Yield: $7.14 \mathrm{~g}(56 \%)$. ${ }^{1} \mathrm{H}$ NMR $\left(400 \mathrm{MHz}\right.$, DMSO- $\left.d_{6}, \delta\right): 8.70\left(\mathrm{~d}, J=4.7 \mathrm{~Hz}, 4 \mathrm{H}, \mathrm{H}^{3}, \mathrm{H}^{3}\right.$ ) $), 8.60(\mathrm{~d}, J=$ $7.8 \mathrm{~Hz}, 4 \mathrm{H}, \mathrm{H}^{6}, \mathrm{H}^{6 ”}$ ), 7.99 (m, $\left.J=7.8,1.9 \mathrm{~Hz}, 4 \mathrm{H}, \mathrm{H}^{5}, \mathrm{H}^{5 ”}\right), 7.98$ (s, 4H, $\mathrm{H}^{3}, \mathrm{H}^{5}$ ), $7.49\left(\mathrm{~m}, J=7.7,4.7,1.1 \mathrm{~Hz}, 4 \mathrm{H}, \mathrm{H}^{4}, \mathrm{H}^{4 \prime}\right), 4.37$ (t, $\left.J=4.8 \mathrm{~Hz}, 4 \mathrm{H}, \mathrm{CH}_{2} \mathrm{Otpy}\right)$, $3.83\left(\mathrm{t}, J=4 \mathrm{~Hz}, 4 \mathrm{H}, \mathrm{CH}_{2} \mathrm{CH}_{2} \mathrm{Otpy}\right), 3.55\left(\mathrm{~s}, \mathrm{CH}_{2}\right)$.

\section{Functionalization assay}

The functionalization of the synthesized PEG(tpy $)_{2}$ is assessed by titration of $15 \mu \mathrm{M}$ PEG(tpy) $)_{2}$ solution in 0.1M MES (pH 5.5) with $\mathrm{CoCl}_{2}$ and recording UVVIS spectra. A stop in increase in intensity of peaks at $305 \mathrm{~nm}$ and $315 \mathrm{~nm}$ is indicative for saturation of all terpyridine moieties. The concentration $\mathrm{CoCl}_{2}$ is directly equivalent to the concentration terpyridine. The functionalization is $92 \%$.

\section{Sample preparation}

Samples are prepared from aqueous stock solutions of $30 \mathrm{wt} \%$ PEG(tpy) $30 \mathrm{wt} \% \mathrm{SiO}_{2}$ nanoparticles $(\mathrm{R}=10 \mathrm{~nm})$ and $0.1 \mathrm{M} \mathrm{CoCl}_{2}$; we first dilute the silica stock solution in an appropriate amount of water, subsequently add PEG(tpy) stock solution, and allow the polymers to adsorp onto silica particles. Finally, we add the $\mathrm{CoCl}_{2}$ solution; in all our samples the molar ratio of tpy: $\mathrm{Co}(\mathrm{II})$ was 
kept constant at 2:1. The samples are mixed vigorously and allowed to equilibrate for at least $48 \mathrm{hrs}$ before measurements.

\section{Dynamic light scattering (DLS)}

The DLS measurements are performed using an ALV goniometer and correlator equipped with a $632.8 \mathrm{~nm}$ JDSU 430N laser and avalanche photodetector (Exelitas Technologies). As the viscosity of some samples is very high, we ensure proper averaging through continuous rotation of the samples at $0.025 \mathrm{rpm}$. To correct for the forced decorrelation imposed by the sample rotation, we measure a strictly non-ergodic reference sample, composed of a highly crosslinked PDMS rubber. Measurements are performed at an angle of $90^{\circ}$.

The measured intensity correlation functions $g_{2}(t)-1$, assumed to go from 1 to 0 , are fitted to a double exponential, of the form:

$$
g_{2}(t)-1=(1-A) \exp \left(-\frac{t}{\tau_{1}}\right)+A \exp \left(-\frac{t}{\tau_{2}}\right)^{\alpha}
$$

in which $A$ is the decorrelation plateau for the first, fast, relaxation mode, $\tau_{1}$ and $\tau_{2}$ are the characteristic relaxation times for the fast and slow relaxation process, respectively, and $\alpha$ is the stretch exponent for the slow relaxation process. This function is fitted to the experimental data using a least-sum-ofsquares regression. While the first, fast, process, is well-fitted with a simple exponential in all cases, indicative of a single relaxation time, the stretch exponent for the slow relaxation process is found to be between 0.33 and 1.28 , indicative of a relatively broad relaxation spectrum for this mode.

\section{Rheology}

Rheological properties are measured on a TA Discovery HR-3 Hybrid rheometer, equipped with a $40 \mathrm{~mm}$ parallel plate geometry and an aluminum Peltier plate, a solvent trap is used to minimize evaporation. All measurements are performed at $23.5^{\circ} \mathrm{C}$. The default protocol consists of a $60 \mathrm{~s}$ preshear step at $10 \mathrm{~s}^{-1}$ shear rate, to erase the effects of sample loading, after which the sample is equilibrated for $300 \mathrm{~s}$ before measuring. Frequency sweeps are recorded at $5 \%$ strain, and increasing frequency between of 0.01 and $100 \mathrm{rad} / \mathrm{s}$. Flow curves are recorded in a shear rate range of 0.01 to $100 \mathrm{~s}^{-1}$, averaging over $30 \mathrm{~s}$ and equilibrating 5 $\mathrm{s}$ between subsequent shear rates to demonstrate the self-healing we again 
equilibrate the samples for $300 \mathrm{~s}$ before loss and storage moduli are measured in oscillatory mode for $300 \mathrm{~s}$ at $5 \%$ strain and $10 \mathrm{~Hz}$ to establish a baseline. Subsequently, the samples are broken through $120 \mathrm{~s}$ rotation at $10 \mathrm{~s}^{-1}$, upon which we cessate the flow and follow the sample recovery under constant oscillation at $10 \mathrm{~Hz}$ and $5 \%$ strain.

\section{Small-angle X-ray scattering}

Small-angle X-ray scattering measurements were performed on a SAXSLAB GANESHA 300 XL SAXS system equipped with a GeniX 3D Cu Ultra Low Divergence micro focus sealed tube source producing X-rays with a wavelength $\lambda=1.54 \AA$ at a flux of $1 \times 108 \mathrm{ph} \mathrm{s}^{-1}$ and a Pilatus $300 \mathrm{~K}$ silicon pixel detector with $487 \times 619$ pixels of $172 \mu^{2}$ in size placed at a sample-to-detector distance of $463 \mathrm{~mm}$ and $1513 \mathrm{~mm}$ to access a $q$-range of $0.003 \leq \mathrm{q} \leq 0.6 \AA^{-1}$ with $q=$ $4 \pi / \lambda(\sin \theta / 2)$. Silver behenate was used for calibration of the beam center and the q-range. Samples were contained in $1 \mathrm{~mm}$ quartz capillaries (Hilgenberg GmbH, Germany). The two-dimensional SAXS patterns were azimuthally averaged to obtain one dimensional SAXS profiles. Hereafter, the scattering contribution of the solvent and quartz cell was subtracted from the scattering curves of the samples. The SAXS scattering profiles were analyzed using the software package SasView. SAXS curves for $10 \%$ silica with and without coordination polymer and $\mathrm{Co}(\mathrm{II})$ are shown in Fig.5.S1.

\subsection{Results and Discussion}

To form a percolating network from supramolecular building blocks, the primary units, or tectons, must allow the formation of more than two bonds, otherwise only chain extension occurs [14]. Multivalent tectons may be realized chemically by using, for example, branched polymers [15], dendrimers [16], or graft co-polymers [17]; here we choose a non-synthetic approach, using noncovalent inter-actions. We create multivalent tectons through physical adsorption, by means of hydrogen bonding, of bifunctional polymers, $\alpha, \omega$ - 


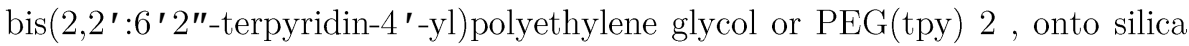
nanospheres. The polymers used here have a radius of gyration of $R_{g} \approx 4 \mathrm{~nm}$. Onto a single spherical nanoparticle with a hydrodynamic radius of $10 \mathrm{~nm}$, approximately 75 polymer chains can adsorb, resulting in a valency of approximately 150 . The surface area of the silica nanoparticles used here is approximately $130 \mathrm{~m}^{2} \mathrm{~g}^{-1}$, assuming an adsorbed amount of approximately 1 $\mathrm{mg} / \mathrm{m}^{-2}[18], \quad 0.1 \mathrm{~g}$ silica particles provides surface area for the adsorption of $\approx 10 \mathrm{mg}$ PEG. This means that in all our samples, we have excess PEG(tpy) with respect to the amount required to coat all of the silica nanoparticles. We thus expect supramolecular network formation in which the colloidal tectons are not directly connected, but are connected through chains of bifunctional polymer (see Fig5.1). We first fi $\mathrm{x}$ the PEG(tpy) $)_{2}$ concentration at $10 \mathrm{wt} \%$, and vary the amount of silica particles added. At low nanoparticle concentrations, a liquid-like material is found, as evidenced by rheological measurements (Fig.5.2a). As the silica particles form the cross-links in the gel, the modulus increases with the concentration of particles in the net-work (Fig.5.2d). When the silica concentration exceeds approximately $5 \mathrm{wt} \%$, the modulus increases steeply, and a frequency dependence typical of a viscoelastic liquid, with a crossover in the loss $\left(G^{\prime \prime}\right)$ and storage $\left(G^{\prime}\right)$ moduli, is found, indicating network formation. At $10 \mathrm{wt} \%$ of silica nanoparticles, the modulus is increased by five orders of magnitude with respect to the nanoparticle-free solutions. Also the low-shear viscosity, determined from rate-dependent viscosity measurements as shown in Fig.5.2b, rapidly increases with increasing nanoparticle concentration (Fig.5.2e). Both the steep increase in modulus and low-shear viscosity indicate efficient formation of a transient network. From these measurements, we can also extract the mechanical relaxation time of the supramolecular networks, which indicates the typical time scale over which imposed stresses are relaxed. 

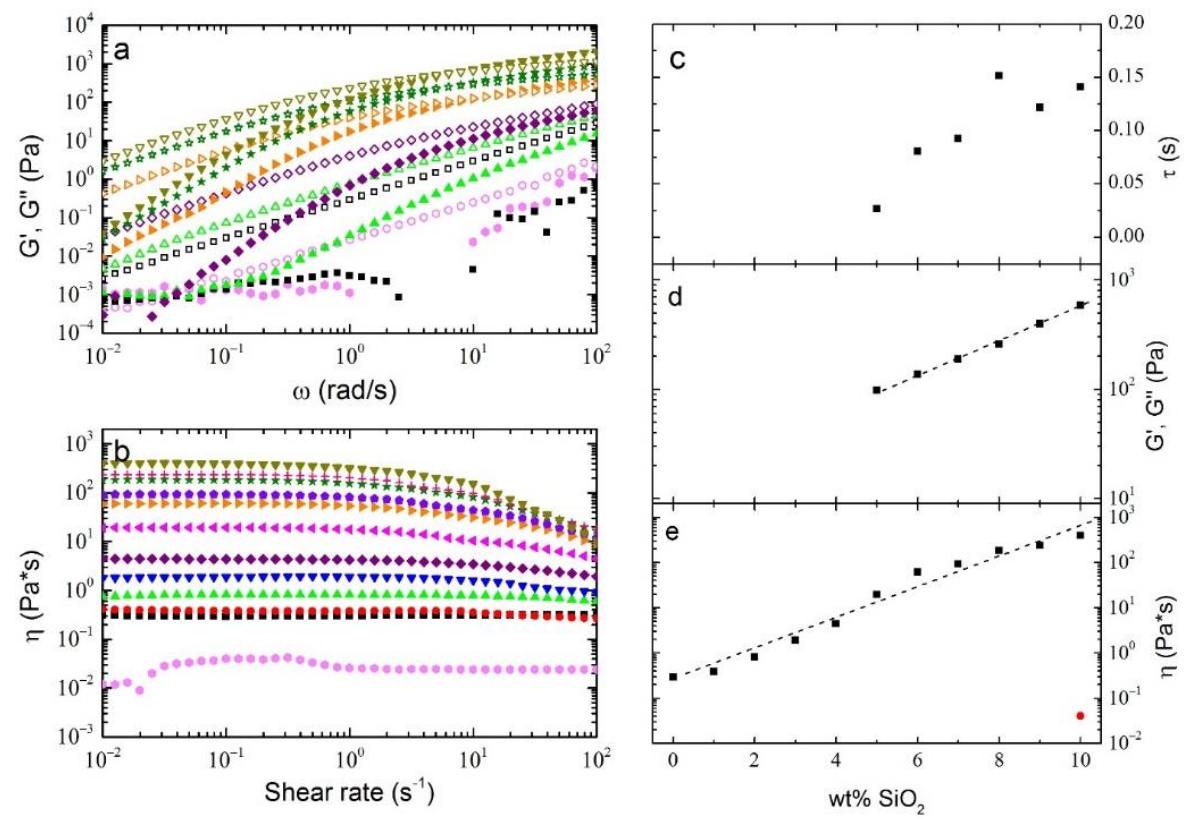

Figure 5.2. a) Frequency sweeps of samples containing 10 wt\% PEG(tpy) 2 with Co(II) and 0 (squares), 5 (hexagons), and $10 \mathrm{wt} \%$ (left triangles) silica, and a sample containing 10 wt\% silica without Co(II) (right triangles). Full symbols represent storage moduli ( $\left.G^{\prime}\right)$, open symbols represent loss moduli ( $G$ "). b) Flow curves of samples containing 0 (squares), 2 (circles), 4 (upward triangles), 6 (downward triangles), 8 (diamonds), and 10 wt\% (left triangles) silica, and a sample containing 10 wt\% silica without Co(II) (right triangles). c) Relaxation times of the networks, from the $\mathrm{SiO} 2$ (squares) and solids (circles) weight series, as obtained from frequency sweeps. d) Shear elastic modulus at the crossover frequency from the SiO 2 (squares) and solids (circles) weight series. The dashed line is a guide to the eye and indicates the exponential regime. The drawn line is a fi t to the experimental data. e) Low-shear viscosity as a function of silica concentration (squares) or solids concentration (circles). The red triangle represents a sample containing 10 wt\% SiO 2 and 10 wt\% PEG(tpy) 2 without Co(II). The dashed lines are guides to the eye and indicate the exponential regime. 
The fact that our systems show a characteristic relaxation time originates from the transient nature of the bonds that form the network. We find a weak dependence of the relaxation time on silica concentration (Fig5.2c), which is probably due to subtle changes in network topology [19]. Interestingly, with increasing silica concentration, we find an exponential growth in both modulus and low-shear viscosity, which are proportional to each other in the Maxwell model through the relaxation time, indicating that the mechanical properties of these materials are highly tunable over several orders of magnitude. We also investigate these effects at the microscale, through dynamic light scattering (DLS) measurements. In our samples, the scattering of the nanoparticles always exceeds the inherent scattering of the polymer chains; the observed relaxation dynamics thus reflect the local motions of the cross-links in the network.
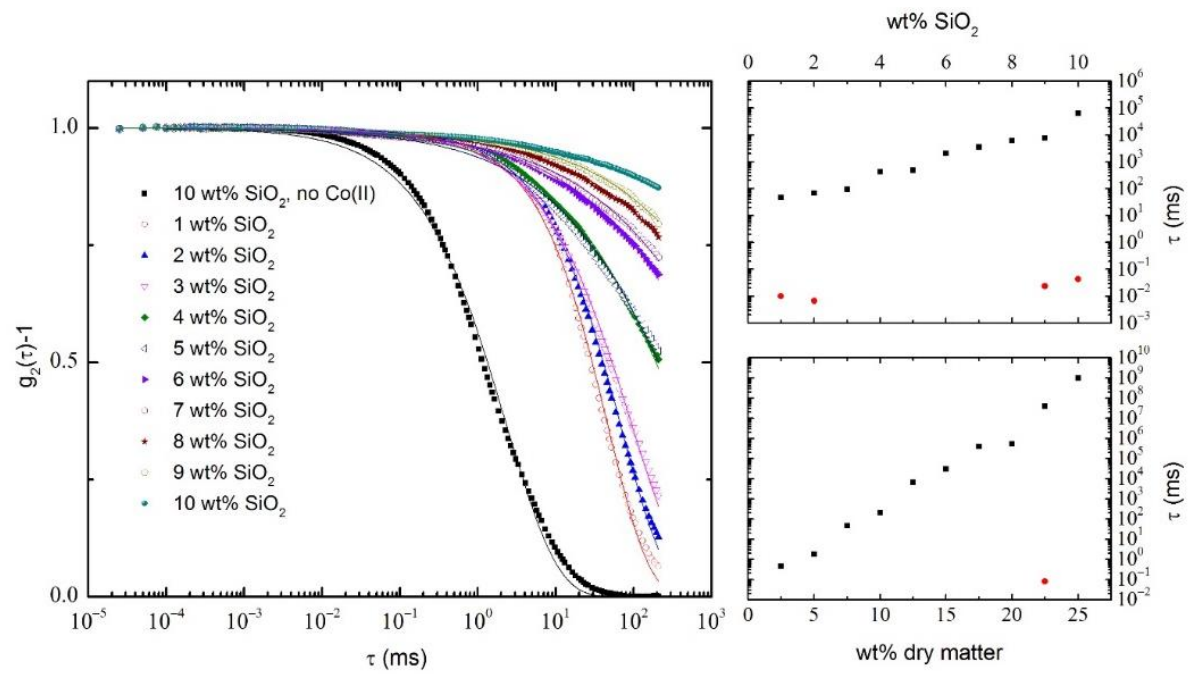

Figure 5.3. a) Corrected correlation curves of samples containing between 0 wt\% and $10 \mathrm{wt} \%$ silica (symbols), $10 \mathrm{wt} \%$ PEG(tpy) 2 and Co(II). The lines represent fits to the data. b) Relaxation times obtained from fitting to corrected data from the silica weight series. The triangles represent $\tau_{1}$, the squares represent $\tau_{2} . c$ ) Relaxation times obtained from fi tting to corrected data from the solids weight series. The triangles represent $\tau_{1}$, the squares represent $\tau_{2}$. 
Due to the high stiffness of the gels, leading to a loss of ergodicity at experimentally accessible time scales, we impose forced decorrelation and ensemble-averaging through rotation of the sample. Subsequently, the correlation functions, corrected for the forced decorrelation, are fitted to a double-stretched exponential function to extract two distinct relaxation times (see Appendix). The short relaxation time $\tau_{1}$ corresponds to the local vibrations of particles around their position in the network. At longer time, particles can escape from their local cages and translate through the network, characterized by a longer relaxation time $\tau_{2}$. The correlation curves show a strong influence of the silica concentration on the decor-relation of the network (see Fig.5.3a). In most cases, the decorrelation due to translation is so strong that no reliable value for the short-time vibrational relaxation could be extracted. $\tau_{1}$ is practically independent of the overall silica concentration, as it only reflects highly local fluctuations during which the particles do not experience the surrounding polymer network. By contrast, the timescale for translation, $\tau_{2}$, increases exponentially with the weight fraction of silica and varies, within our experiments, over four orders of magnitude (Fig.5.3b). Surprisingly, this suggests that the microscopic dynamics slow down much stronger with increasing cross-link density than the macroscopic properties. In macroscopic rheology, where we disentangle the modulus from the mechanical relaxation time, we mainly measure the dissociation of metal-ligand complexes, which provide mechanical stability to the network. By contrast, in the DLS measurements, we measure the diffusion of the silica nanoparticles, which is governed by the sample viscosity. Indeed, the viscosity of the samples, by approximation the product of modulus and relaxation time, increases exponentially, as does the slow relaxation time from DLS. We also investigate the effect of the overall solids concentration, maintaining a 1:1 weight ratio of nano-particles to polymer. Here, we also find a strong dependence of both modulus and viscosity, showing a similar dependence on solids concentration as on overall silica concentration with fixed amount of polymer (Fig.5.2d,e, blue circles), while the macroscopically determined mechanical relaxation time is nearly independent of overall concentration (Fig.5.2c). The shear modulus versus total solids concentration c can be well described by an expression, which 
assumes a power-law dependence of the modulus upon exceeding the critical gel concentration: $G^{\prime} \propto\left(c-c_{g e l}\right)^{\alpha}$; a fit to the data (drawn line in Fig.5.2d) reveals a minimum concentration of total solids of $4.5 \mathrm{wt} \%$ to achieve gelation, and a power-law exponent of 2.5. Again, these data are confirmed by the microscopic relaxation time measured using DLS (Fig.5.3c). Due to the transient nature of both types of bonds in these networks, the hydrogen bonds between polymer and nanoparticle and the coordination bonds that interconnect the multivalent tectons, these materials are expected to self-heal when the network structure is damaged. After disrupting the network structure of a strong gel through intense rotational shear, we observe recovery of the elasticity, tending towards its original value within several tens of minutes; this process can be repeated several times and is reversible (see Fig.5.4). Mainly the weakest bonds in the network, formed by the coordination complex between terpyridine and $\mathrm{Co}(\mathrm{II})$ ions, will be disrupted by the rotation, and because of their reversibility the network structure is able to recover.

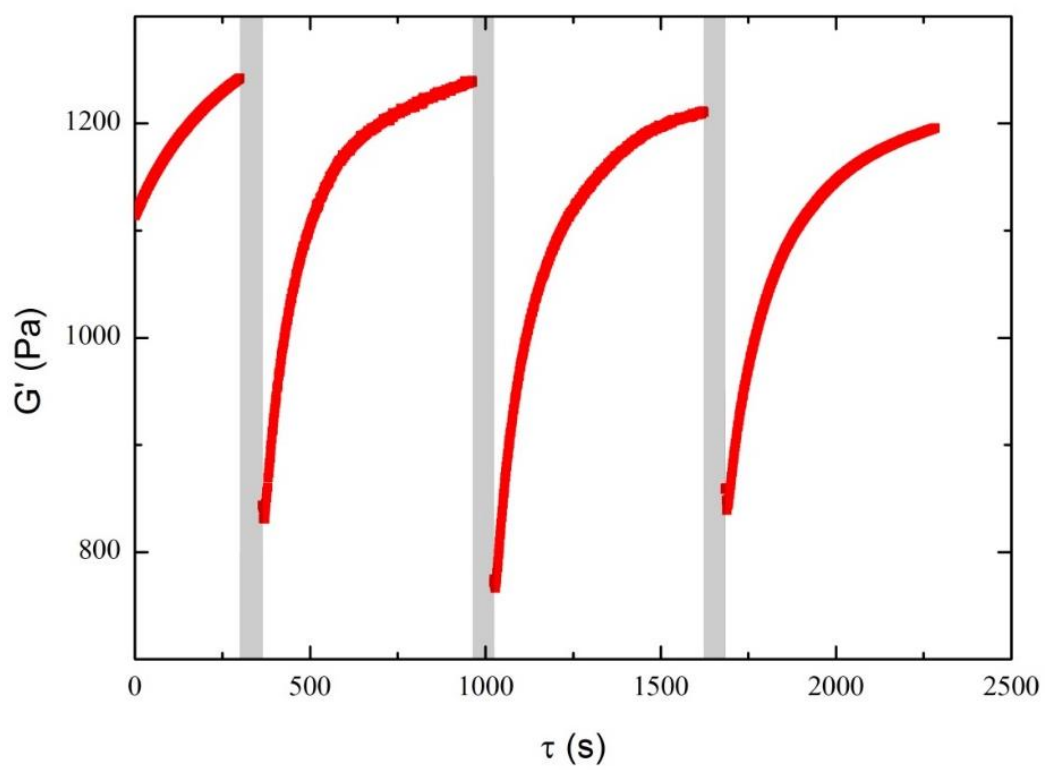

Figure 5.4. Recovery of the storage modulus in time. The gray areas represent breaking of the sample by rotation and are not plotted at real-time scale. 
Interestingly, we observe that the rate at which the network recovers decreases with each repeating cycle, as indicated by the decreasing slope of the initial recovery of the storage modulus. This suggests that some changes to the stronger bonds, those between polymer backbone and nanoparticle surfaces, occur, which may recover to their equilibrium state upon prolonged waiting. Finally, to assess the homogeneity of the nanoparticle-polymer composites, we study the network structure through small-angle X-ray scattering (SAXS). We find no significant differences between samples composed of nanoparticles alone, or a network including polymer, particles and $\mathrm{Co}(\mathrm{II})$ ions over the entire accessible range of scattering vectors. The minima in scattered intensity remain clearly visible in the scattering of the networks and both curves can be well fitted with the same form factor for polydisperse hard spheres, with a radius of $\mathrm{r}=7.9 \mathrm{~nm}$ (See Fig.5.S1, Supporting Information). These data highlight that the nanoparticles remain well dispersed in the polymer network, resulting in very homogeneous, and optically transparent, composite hydrogels.

\subsection{Conclusions}

We have shown a strategy to create multivalent colloidal building blocks, formed through physical interactions between terpyridine-bearing polymer chains and nano-particle surfaces, which can subsequently be assembled through orthogonal supramolecular interactions into homogeneous and optically transparent physical hydro-gels. While we use an orthogonal combination of hydrogen bonding and metal co-ordination interactions, this strategy can be easily extended to the wide variety of specific supra-molecular interactions available to us today. The use of purely physical and orthogonal interactions to assemble self-healing materials holds great promise for the creation of new, responsive materials in which the mechanical and optical properties can be tuned in a synthesis-free approach. This gives great flexibility and versatility to create materials with precisely defined structure and functionality. 


\subsection{References}

1. Foo, S.Y., et al., Vascular effects of a low-carbohydrate highprotein diet. Proceedings of the National Academy of Sciences, 2009. 106(36): p. 15418-15423.

2. Seiffert, S. and J. Sprakel, Physical chemistry of supramolecular polymer networks. Chemical Society Reviews, 2012. 41(2): p. 909-930.

3. Glassman, M.J., J. Chan, and B.D. Olsen, Reinforcement of Shear Thinning Protein Hydrogels by Responsive Block Copolymer Self-Assembly. Advanced Functional Materials, 2013. 23(9): p. 1182-1193.

4. Peng, F., et al., Redox-Responsive Gel-Sol/Sol-Gel Transition in Poly(acrylic acid) Aqueous Solution Containing Fe(III) Ions Switched by Light. Journal of the American Chemical Society, 2008. 130(48): p. 16166-16167.

5. Montembault, A., C. Viton, and A. Domard, Physico-chemical studies of the gelation of chitosan in a hydroalcoholic medium. Biomaterials, 2005. 26(8): p. 933-943.

6. Lemmers, M., et al., Multiresponsive Reversible Gels Based on Charge-Driven Assembly. Angewandte Chemie International Edition, 2010. 49(4): p. 708-711.

7. Pham, T.T.H., et al., Multi-responsive physical gels formed by a biosynthetic asymmetric triblock protein polymer and a polyanion. Soft Matter, 2013. 9(37): p. 8923-8930.

8. Guillet, P., et al., Connecting micelles by metallo-supramolecular interactions: towards stimuli responsive hierarchical materials. Soft Matter, 2009. 5(18): p. 3409-3411.

9. Gaharwar, A.K., et al., Photocrosslinked nanocomposite hydrogels from PEG and silica nanospheres: Structural, mechanical and cell adhesion characteristics. Materials Science and Engineering: C, 2013. 33(3): p. 1800-1807. 
10. Schmidt, G. and M.M. Malwitz, Properties of polymernanoparticle composites. Current Opinion in Colloid \& Interface Science, 2003. 8(1): p. 103-108.

11. Filippone, G. and M. Salzano de Luna, A Unifying Approach for the Linear Viscoelasticity of Polymer Nanocomposites. Macromolecules, 2012. 45(21): p. 8853-8860.

12. Asoh, T.-A., et al., Fabrication of Self-Healable Hydrogels through Sol-Gel Transition in Metallo-supramolecular Aqueous Solution by Aeration. Macromolecular Chemistry and Physics, 2013. 214(22): p. 2534-2539.

13. Zhang, H., et al., Synthesis and fluorescent properties of a novel europium(III) complex with terpyridine-capped poly(ethylene glycol). Journal of Rare Earths, 2012. 30(7): p. 705-708.

14. Koohmareh, G.A. and M. Sharifi, Synthesis, Characterization and Coordination Behavior of Zinc Supramolecular Polyurethane End-Capped with Terpyridine. Designed Monomers and Polymers, 2010. 13(2): p. 123-129.

15. Buwalda, S.J., P.J. Dijkstra, and J. Feijen, Poly(ethylene glycol)poly(L-lactide) star block copolymer hydrogels crosslinked by metal-ligand coordination. Journal of Polymer Science Part A: Polymer Chemistry, 2012. 50(9): p. 1783-1791.

16. Smith, D.K., Dendritic Gels-Many Arms Make Light Work. Advanced Materials, 2006. 18(20): p. 2773-2778.

17. Liu, R., et al., Cationic Temperature-Responsive Poly(Nisopropyl acrylamide) Graft Copolymers: from Triggered Association to Gelation. Langmuir, 2008. 24(14): p. 7099-7106.

18. Fleer, G., et al., Polymers at Interfaces. 1993, London: Chapman and Hall.

19. Skrzeszewska, P.J., et al., Physical gels of telechelic triblock copolymers with precisely defined junction multiplicity. Soft Matter, 2009. 5(10): p. 2057-2062. 


\subsection{Appendix}
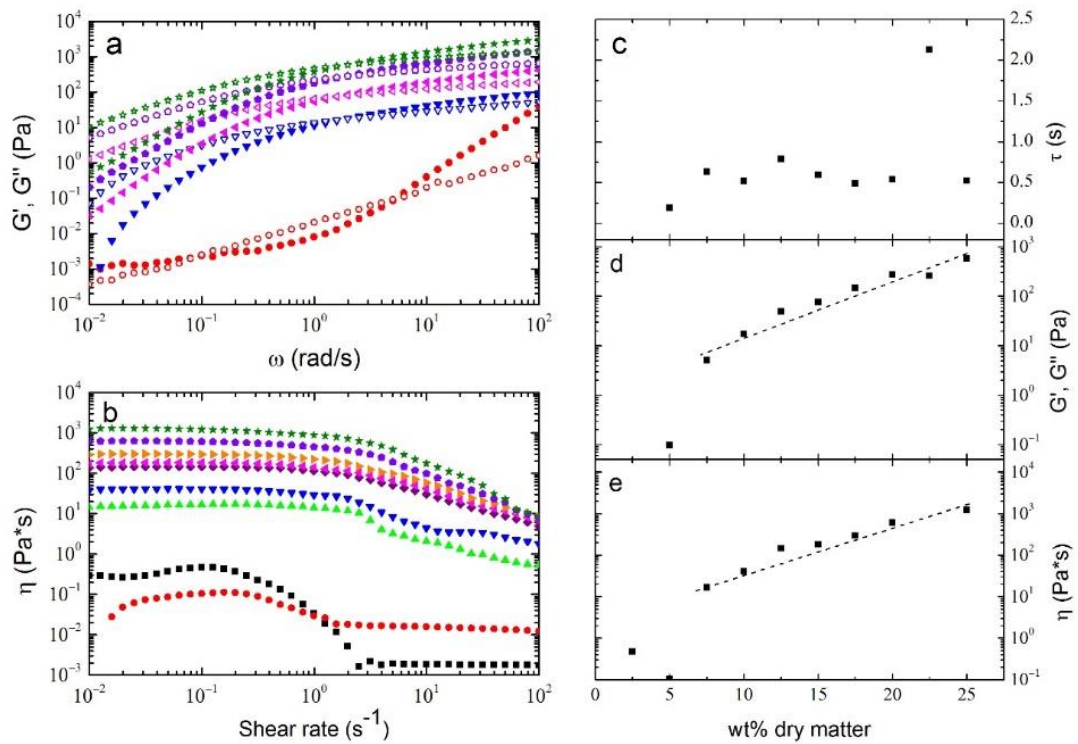

FigureA1. a) Frequency sweeps of networks containing 5, 10, 15, 20, and 25 wt\% dry matter. Symbols correspond to those in Figure S2b. Full symbols represent loss moduli $\left(G^{\prime}\right)$, open symbols represent storage moduli ( $\left.G^{\prime \prime}\right)$. b) Flow curves of networks containing 2.5 wt\% (squares), 5 wt\% (circles), 7.5 wt\% (upward triangles), $10 w t \%$ (downward triangles), $12.5 w t \%$ (diamonds), $15 w t \%$ (left triangles), 17.5 wt\% (right triangles), 20 wt\% (pentagons) and $25 w t \%$ (stars) dry matter. c) Relaxation times of the networks, obtained from frequency sweeps. d) Shear elastic modulus at the crossover frequency. The dashed line is a guide to the eye. e) Low-shear viscosity as a function of overall solids concentration. The dashed line is a guide to the eye. 




Figure A2. Angle dependent X-ray scattering from MAXS and ESAXS experiments. Measurements are performed on a sample containing dispersed silica particles, and a sample containing a network through coordination of Co(II). 


\section{CHAPTER 6}

General Discussion 
Supramolecular polymeric networks are a class of widely investigated materials built from polymeric macromolecules organized by transient and, in most cases, physical (non-covalent) interactions. The non-permanent character of the bonds gives such networks an advantage in the field of applied science as this renders them stimuli-responsive and self-healing. In contrast to their permanently cross-linked equivalents they are dynamic and reversible by nature, and thus form excellent candidates for broad use in medicine, as drug delivery systems, in tissue engineering, cell-culture research, bio-sensing or wound treatment, and in industry as structural adhesives, paint thickeners, binders, etc. To achieve specific properties on demand, however, there is a great need to investigate the fundamental physicochemical properties of supramolecular assemblies, linking microstructure and bond dynamics to their macroscopic properties; however, this understanding is far from complete.

In the last decades, a wide variety of innovative supramolecular systems has been described; yet, there are few systematic studies that aim to unravel the physics of supramolecular ordering and gelation itself. This is due to the richness and the complexity of the supramolecular assembly pathways, among which we can distinguish basic step-by-step association of unimers (so-called multistage open association MSOA) ${ }^{[1]}$, but also helical growth ${ }^{[2]}$ or beta-sheet formation, stacking, clustering and crystallization of the transient crosslinks.

The position and nature of the gelation point remains, an ill-understood phenomenon. In a recent review paper on the physical-chemistry of supramolecular networks, a discussion is presented of linear-chain systems that follow the MSOA pathway of polymerization, e.g. describing the dependency on monomer concentration ${ }^{[3]}$. Gelation occurs then by the increase of chain length that, eventually, will lead to the formation of entanglements that slow down the solution kinetics. Other systems, which do not entangle, associate in order to form supramolecular nodules, which result in the evolution of percolated networks above a critical concentration $c^{*}$. Most works, however, limit their descriptions to a phenomenological picture. This situation is exacerbated by the fact that the association mechanism and kinetics and the percolation point itself can be easily disturbed by many external factors because the physical 
forces that provide the material with cohesion are in the range of $k T$. This and many other factors make the formation of supramolecular networks sensitive to environmental stimuli and thus not easily described by simple approaches.

We can recall two theoretical strategies that try to model the route of gelation. What about this? The route of gelation can be modelled by basically two theoretical strategies. The first, introduced by Flory and Stockmayer [4, 5], proposes a mean-field lattice model which describes gelation as a statistical process of randomly chosen associating moieties. The model predicts phase diagrams with respect to concentration and temperature by accounting for chain topology, such as rings and loops, and the influence of polymer-polymer and polymer-solvent interactions. The model was later extended by Semenov and Rubinstein ${ }^{[6]}$ who introduced excluded volume. They predicted the possibility of phase-separation driven by supramolecular association of assembling moieties in marginal and theta-solvents and by that they showed that the percolation doesn't take place in a continuous phase transition manner.

A second approach, introduced in this context by de Gennes, describes the gelation as a result of critical percolation ${ }^{[7]}$. This is based on the assumption that the polymerization occurs by random connections made to form polymeric clusters. Based on the idea that monomers occupy sites on a periodic and regular lattice, which forms bonds between the nearest neighbors with the probability $\mathrm{p}$, the percolation approach predicts a critical fraction of bonds which need to be made for a system-spanning cluster to emerge.

Considerations regarding gelation are naturally followed by questions regarding the microstructure of the supramolecular polymer networks formed. By contrast to conventional covalently cross-linked networks, supramolecular assembly can exhibit a much richer complexity. This can for example be due to the functionality of association motifs to form directional bonds with a well-defined valency. Additionally, the associative moieties can form conformational structures that result in further structural complexity and domain formation at larger length scales. These domains can be a result of local crystallization, stacking of functional moieties, clustering, bundling or even formation of 
complexed colloidal structures ${ }^{[3]}$. At even larger scales, this can manifest in micro-phase separation, for example into regions rich in crosslinks and those consisting of free chains only. While such large scale heterogeneities result in reduced mechanical performance in covalent networks, it can surprisingly reinforce the mechanics of supramolecular networks ${ }^{[8,9]}$. We can speculate that structurally, such separated gels, may resemble polymeric composites, where two components with different stiffness are mixed and materials with synergistic properties can result, when properly designed. Yet, why this would be the case for supramolecular gels and not for their covalent counterparts is unclear and thus it brings a great need for systematic research that would describe the pathway of formation of such nano-structures and their influence on the macroscopic gel performance.

A third crucial question regards the intrinsic dynamics of gels, which are equally important in determining their mechanical properties as their microstructure is. Natural characteristic timescales to consider in these systems are those related to association and dissociation and those relaxation processes that deal with relaxation of the polymeric chain/monomer/cluster. Literature is rich in research focusing on dynamics occurring within a supramolecular gel at the molecular scale. However, how this translates into dictating structure and ensuing macroscopic mechanics remains challenging to unravel.

In this thesis the formation of supramolecular networks from end-functionalized linear polymers has been the main subject. Chapters 2 to 4 are devoted to fundamental studies on the phenomenon of gelation itself, in which the role of connectivity in the gelation processes and the origins of kinetically arrested structures are addressed.

In Chapter 2 we revealed new insights into the complex microscopic dynamics of transient networks, assembled by hydrophobic forces. Using light scattering experiments we show how these materials exhibit complex multimodal relaxation spectra. To shed light on the nature of such relaxation processes we systematically changed the network architecture by gradually reducing the network connectivity while keeping the polymer concentration constant. This 
strategy allows us to disentangle the roles of concentration and connectivity on the dynamic modes of these systems.

In Chapters 3 and Chapter 4 we experimentally explored the pathways of network formation from telechelic polymers association by means of metalligand complexation. Interestingly, while some networks exhibit near-ideal Maxwellian behavior, as expected for transient networks, we find certain cases where we observe scale-free critical mechanics. To date this latter behavior was only identified close to a covalent percolation transition. The critical behavior observed for these end-functional self-assembled polymer networks, however, is robust to changes in concentration, temperature and crosslinking degree. Our studies show that such a self-organized and robust critical state is the results of arrested phase separation that kinetically traps the network-forming system at its percolation point. The system thus remains trapped in a critical state resulting in robust power-law scaling of shear and relaxation moduli. We also show how this state depends sensitively on the relaxation kinetics of the nodes by demonstrating an intermediate case where initial critical behavior slowly relaxes over the course of several days to the ideal linear Maxwell case. With our research we highlight the complex pathway where self-assembling systems reach their equilibrium ground state, involving persistent and long-lived kinetically arrested states which give rise to unusual mechanics and highly heterogeneous spinodal structures.

Chapter 5 brought us towards more applicable materials where we develop a highly tunable composite network based on orthogonal supramolecular interactions. For such a design we generate multivalent nanoparticle tectons, which are subsequently linked together into network structures, using metalcoordination interactions. Materials built this way are highly tunable with moduli and viscosities spanning many orders of magnitude.

In the remainder of this chapter, we focus on some unresolved and outstanding questions regarding the physical chemistry and properties of supramolecular networks and we will discuss some preliminary data obtained in our efforts to resolve them. 


\section{1. $\quad$ Phase separation \& mechanical properties}

A number of previous studies focused on hydrogels built using metal-ligand interactions. In this thesis we limited our studies to a linear telechelic building block; however, most of the reported gels are built from more complicated polymeric designs. Rossow and Seiffert presented a system based on 10kDa starshaped poly(ethylene glycol) functionalized with terpyridine moieties at the chain extremities, crosslinked with divalent $\mathrm{Mn}, \mathrm{Zn}$ and Co metal ions ${ }^{[10,11]}$. Gels formed in this way, in various solvents, exhibited clear Maxwellian rheology with plateau moduli, for $\mathrm{Zn}$ and $\mathrm{Co}$ ions, of around $10 \mathrm{kPa}$ for a concentration of $200 \mathrm{~g} / 1$. Such high moduli can be a result of insufficient equilibration time as the measurements reported by Rossow and Seiffert were performed immediately after gelation occurred, while our experiments indicate that the mechanical properties in these systems can evolve very slowly, requiring careful experimentation to probe the equilibrium dynamics. Interestingly, while others have reported slight phase separation in samples crosslinked with cobalt metal ions, the clear Maxwellian response, as opposed to the critical phenomena we observe, suggests that a star-like architecture brings additional stability to the formed networks. A similar architecture was studied by Wang et al. ${ }^{[12]}$. In this case the backbone polymer was built from $20 \mathrm{kDa} 8$-armed poly(ethylene glycol) functionalized on 4 arms only and crosslinked with a set of four transition metal ions, namely $\mathrm{Zn}, \mathrm{Co}, \mathrm{Ni}$, and Fe. Also these authors, in analogy to our findings, report the formation of spherical condensed droplets at low concentrations and critical mechanics at higher concentrations. What is striking in the results in all of these studies is that the mechanical moduli appear relatively independent of the molecular architecture. This suggests that it is the phase separated structure that is responsible for the mechanics in these systems, not the molecular structure itself.

Literature also corroborates our findings on the structural evolution of transient networks after passing the gel point ${ }^{[10]}$. FRAP experiments, determining the diffusivity of molecular tracers, revealed a distinct change in diffusivity in metalcrosslinked polymer networks over time. Below the overlap concentration the diffusion rate decelerates systematically with time whereas above it, a 
characteristic jump into higher rates is observed. This implies that these networks indeed evolve from a phase separated, stronger network into an equilibrated and homogeneous state which is much more dynamic in nature.

\subsection{Critical gelation \& structure}

While Chapters 3 to 5 report a detailed study on the phenomena observed in metal-coordinated networks, several unresolved questions remain. In Chapter 3 we addressed the phase-separation driven critical gelation of aqueous solutions of metalorganic assemblies, yet it remains unclear what interactions are at the origin of the higher-order assembly of the metal-coordinated terpyridine moieties. It could be the result of non-homogenous mixing while preparing the gel, where even while applying the metal ion solution with care, local concentration gradients remain. The dropwise dosage of iron and nickel solutions could force strong complexation on the droplet surface and block the diffusion of the ions from the inner part of the droplet. Therefore, there is a need of additional studies that would elaborate more controlled methods of complexation, e.g. using $\mathrm{pH}$ or redox-chemistry to switch the complexes on after full mixing. To control the quenching, hydrolysis of gluconolactone can be used as it supplies the controlled release of protons which acidifies the solution. Yet, even if the phase separation we observe is protocol dependent, its origins cannot be resolved without deeper investigation of the phase separated structure, e.g. to explore if the coordinated terpyridine groups crystallize or stack in an ordered manner, which could be ascertained using wide-angle x-ray scattering. The mesh size of networks we report in Chapter 3 is equal to $160 \mathrm{~nm}$, which is far larger than the radius of gyration of the polymer itself. This was our main indication of phase-separation-driven gelation; these larger structures could be ideally studied using small-angle $\mathrm{x}$-ray or neutron scattering, to determine their inner structure. Finally, a thorough study of the fluctuations of the phase separated structure, exploring a wide range of ligand-metal pairs, using light scattering, could shed new light onto the origins of this critical gelation. 


\subsection{Adhesives}

The materials presented in Chapters 3 - 5 exhibit strong adhesion properties. Polymeric adhesives are under intense investigation as they find applications in paints or as glues and structural adhesives ${ }^{[13,14]}$. In Fig.6.1.a we show an example of how the critical gel built form terpyridine-functionalized telechelics, complexed with Fe(II), adheres strongly to a plastic surface. In Fig. 6.1.b we show preliminary quantitative data of an adhesion measurement, in which a 9.5 $\mathrm{mm}$ diameter glass sphere is brought in contact with a flat glass substrate coated with the critical gel under controlled relative humidity conditions. In this figure positive loads are tensile and negative loads are compressive. The spherical indenter is fixed to a Futek load cell and its motion controlled by a linear actuator (Thorlabs Z825B) connected to a controller (Thorlabs TDC001). The spherical indenter is translated towards the film at at $0.01 \mathrm{~mm} / \mathrm{s}$ until a defined preload force is reached (green symbols), where it is equilibrated for 30s. Subsequently, the indenter is retracted at the same speed, upon which the adhesion force between the glass probe and the polymer film can be measured.
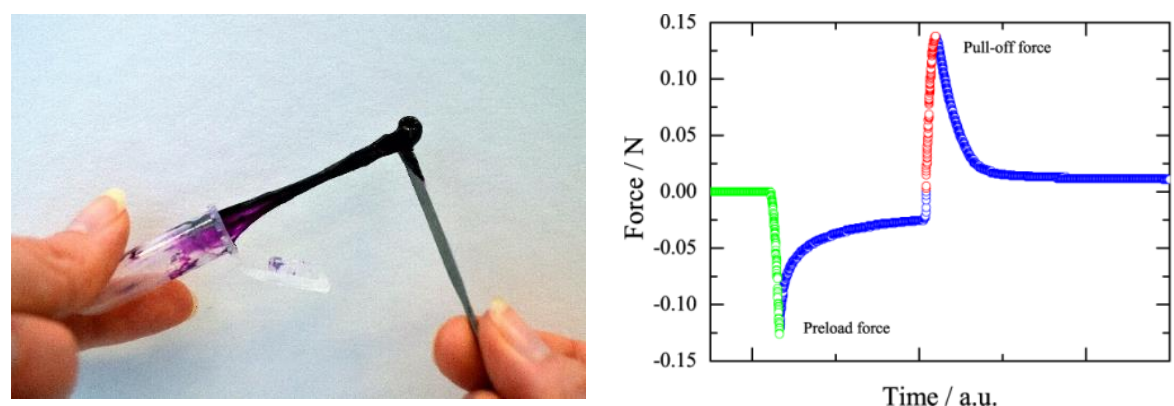

Figure 6.1: (a) Iron based gel formed through arrested phase separation, (b) adhesion experiment with a spherical indentor showing non-linear relaxation processes leading to cohesive failure. 
From the registered curves we can determine the pull-off force which is a measure for the adhesive performance. Interestingly, when the probe is retracted we can observe a peculiar, non-linear relaxation pattern in the adhesive layer. Rather than failing at either interface, we observe strong fibrillation of the adhesive film followed by cohesive failure; indicative of strong adhesion with the glass substrates.

These preliminary results open new paths for future research. A few strategies may be employed to further enhance the adhesive performance, e.g. by increasing the cohesive fracture toughness of the material. This could be accomplished by creating composites with nanoparticles or clays, as discussed in Chapter 5 and formulating layer-by-layer structures, which would introduce another polymeric gel as a scaffold for the metal-organic material. Such a layered design allows maximizing the control over the experiment and provide easy to install samples for any tensile tester. Another solution for increasing the fidelity of adhesion experiments on these materials is proposed by Heinzmann et al. who spreads the supramolecular polymer between two glass, quartz and steel slides upon which it is installed in the experimental setup ${ }^{[15]}$.

\subsection{Telechelic-based double-network composites}

Double-network hydrogels, a special type of interpenetrating polymer networks, have been under intense investigation in the past years. These materials give a promising path for future development of artificial tissues due to their unique mechanical properties. They consist of two independently cross-linked, waterfilled polymer networks that are topologically interlocked. When appropriately designed, the networks create a mechanical synergy, which results in outstanding mechanical properties such as a large extensibility and high strength and fracture toughness. Most of these studies have focused on covalently cross-linked double networks that indeed display impressive performance, however, due to their covalent nature, once prepared the materials cannot be processed nor can internal damage that occurs during large 
deformations be spontaneously healed. Consequently, the fracture toughness is known to deteriorate rapidly as the material is loaded and unloaded repetitively.

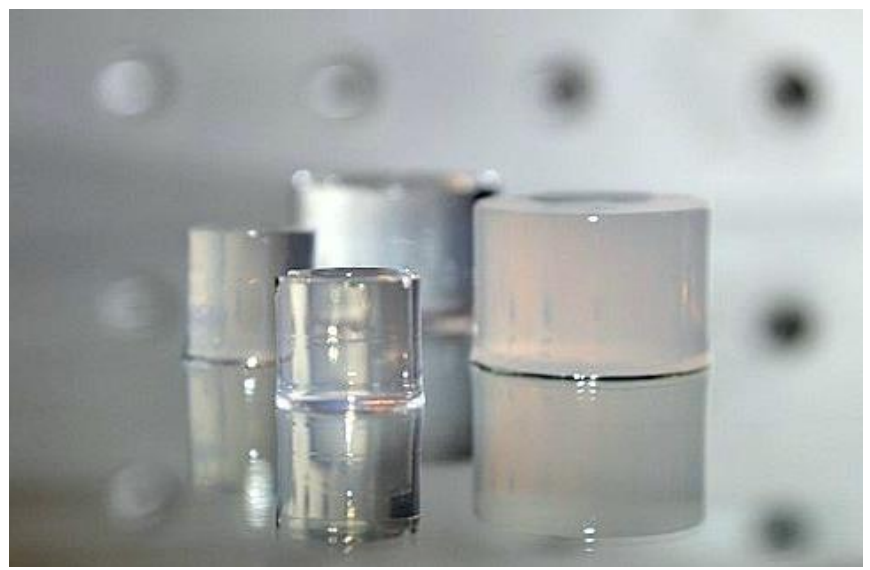

Figure 6.2: Composite networks consisting of hydrophobically modified telechelic polymer as a sacrificial network and a covalent-polymer gel as the scaffold.

We have investigated, in some preliminary experiments, the possibility to use telechelic supramolecular polymer structures as self-healing sacrificial networks to circumvent these constraints. One of the challenges is that mixing polymers with different chemical nature almost invariably leads to phase separation. Thus, the main challenge is to establish conditions for fixing the networks while blocking the tendency for macroscopic spinodal decomposition. This can be achieved by choosing a proper molar ratio between the two networks and by controlling the kinetics of crosslinking. In this project we performed a number of experiments on mixtures of hydrophobically-modified telechelics with various types of covalent and non-covalent networks. In Figure 6.2. we show some different double networks in which a hydrophobic telechelic transient network is interpenetrated with a covalent gel of oligoethylene glycol methacrylate. The samples in the front show no sign of phase separation, as they are optically 
transparent, where the transient network is present at $2.5 \mathrm{wt} \%$ and the covalent methacrylate network at $1 \mathrm{wt} \%$. Upon increasing the concentration of the covalently crosslinked network, we observe turbidity emerging in the samples, which indicates the formation of phase separated structures. Dynamic Mechanical Analysis performed on these samples showed that the phase separation results in materials much weaker than either of the single components, highlighting the importance of controlling the microstructure of such double network materials.
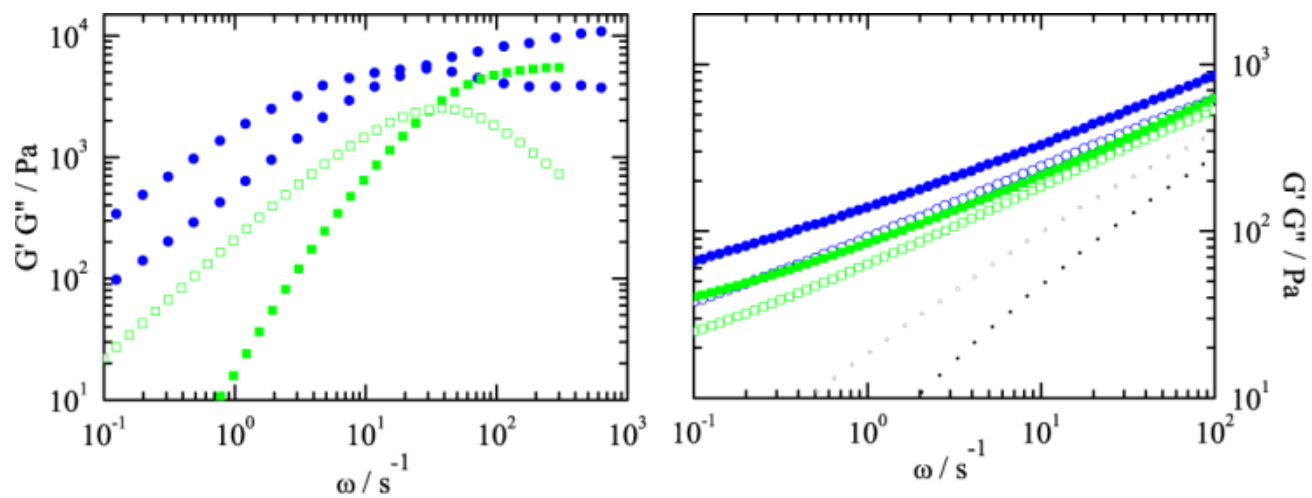

Figure 6.3: Frequency sweep conducted on the composite networks consisting of (a) hydrophobically modified C18PEOC18 PEO and 1wt\% PVA; telechelic polymer is represented by the green squares, and composite by blue circles. (b) Composites built from 10wt\% terpyridine functionalized PEO, enhanced with $1 w t \%$ CNC (green squares) and with 1\% xanthan gel (blue circles). Black dots stands for a 10 wt\% nickel gel without the nano-fillers. G' is represented by closed symbols and $G$ " by open symbols.

Similar cloudiness occurred when using a polyvinyl alcohol gel, however, this time, for a sample containing $1 \%$ of this polymer, we observed an increase in the moduli (Fig.6.3.a). To avoid phase separation, further studies should include playing with the telechelic architecture, or the inclusion of backbone 
charges. Another possibility lies in controlling the crosslinking processes for methacrylic or other covalently crosslinked networks by using photoinitiation under controlled conditions.

The effect of the mutual synergism observed in double network composites can also be achieved by replacing one of the networks with colloidal particles (see Chapter 5). We first coat small colloidal particles with terpyridine end-capped polymers and subsequently, assembled these into composite hydrogels by addition of $\mathrm{Co}(\mathrm{II})$ ions, creating transient metal-coordination linkages between the particles. The mechanics of the formed networks can be tuned over many orders of magnitude and this composite can spontaneously restore its mechanical properties within several minutes after failure.

The same telechelics were also tested with other types of fillers, such as crystalline nanocellulose (CNC), clay platelets and magnetite nanoparticles, or biopolymers such as xanthan fibers or gel networks of flexible polymers. Fig.6.3.b shows an example of preliminary data on metal-coordinated networks filled with nanocellulose and xanthan gum. Also for these fillers we observe a strong increase in mechanical properties, making these systems promising for further study. Of special interest is the ability to add additional tuneability to these materials due to these fillers; for example using their sensitivity to uniaxial shearing (CNC, nano-clay), external magnetic fields (magnetite) and ionic conditions (xanthan gum).

\subsection{Self-oscillating hydrogels}

Finally, we discuss an additional application of terpyridine-based telechelics in self-oscillating hydrogels. This new type of smart material has gained attention in the past few years and is inspired by a chemical oscillating reaction known as the Belousov-Zhabotinsky (BZ) chemical clock. This complex reaction is based on a metal-ion-catalyzed reversal oxidation of malonic acid by an acidic bromate solution, governed by non-equilibrium thermodynamics. The reaction periodically, or chaotically, oscillates, generating time-dependent concentrations 
of the various species. A promising system was proposed by Yoshida et al. which contained self-oscillating structural gels built from the well-known stimuliresponsive polymer poly-(N-isopropylacrylamide) (PNIPAM) grafted with $\mathrm{Ru}$ (bipy)3 moieties ${ }^{[16]}$. The metal ion acts as a catalyst, where the BZ reaction triggers oscillations between $\mathrm{Ru}(\mathrm{III})$ and $\mathrm{Ru}(\mathrm{II})$ states. As those two oxidation states give rise to different association constants for the coordination complex with bipy moieites, the BZ reaction results in periodic volume oscillations of the hydrogel. This design leads to wave-like patterns that trigger an effect of selfwalking, breathing or peristaltic pumping. This makes these materials extremely promising as biomimicking materials. This could be extended by incorporating ruthenium complexes in tough and self-oscillating composite hydrogels based on terpyridine functionalized telechelic polymers (Chapter 5). Assuming that the ruthenium complexation of bare metalorganic polymer can again lead to critical gelation (Chapters 3-4) the composite architecture combined with selfoscillations could provide materials which self-anneal over time. 


\subsection{References}

[1] S. Sugihara, S. Kanaoka, S. Aoshima, Journal of Polymer Science Part A: Polymer Chemistry 2004, 42, 2601-2611.

[2] P. J. Skrzeszewska, F. A. de Wolf, M. W. T. Werten, A. P. H. A. Moers, M. A. Cohen Stuart, J. van der Gucht, Soft Matter 2009, 5, 2057-2062.

[3] S. Seiffert, J. Sprakel, Chemical Society Reviews 2012, 41, 909-930.

[4] P. J. Flory, Journal of the American Chemical Society 1941, 63, 30833090 .

[5] W. H. Stockmayer, The Journal of Chemical Physics 1944, 12, 125131.

[6] A. N. Semenov, M. Rubinstein, Macromolecules 1998, 31, 1373-1385.

[7] P.-G. De Gennes, P. Lafore, J. P. Millot, Journal of Physics and Chemistry of Solids 1959, 11, 105-110.

[8] M. M. L. Nieuwenhuizen, T. F. A. de Greef, R. L. J. van der Bruggen, J. M. J. Paulusse, W. P. J. Appel, M. M. J. Smulders, R. P. Sijbesma, E. W. Meijer, Chemistry - A European Journal 2010, 16, 1601-1612.

[9] D. J. M. van Beek, A. J. H. Spiering, G. W. M. Peters, K. te Nijenhuis, R. P. Sijbesma, Macromolecules 2007, 40, 8464-8475.

[10] T. Rossow, A. Habicht, S. Seiffert, Macromolecules 2014, 47, 64736482 .

[11] T. Rossow, S. Seiffert, Polymer Chemistry 2014, 5, 3018-3029.

[12] R. Wang, M. Geven, P. J. Dijkstra, P. Martens, M. Karperien, Soft Matter 2014, 10, 7328-7336.

[13] C.-J. Wu, J. J. Wilker, G. Schmidt, Macromolecular Bioscience 2013, 13, 59-66.

[14] H. Yuk, T. Zhang, S. Lin, G. A. Parada, X. Zhao, Nat Mater 2016, 15, 190-196.

[15] C. Heinzmann, S. Coulibaly, A. Roulin, G. L. Fiore, C. Weder, ACS Applied Materials $\&$ Interfaces 2014, 6, 4713-4719.

[16] R. Yoshida, T. Sakai, O. Tambata, T. Yamaguchi, Science and Technology of Advanced Materials 2002, 3, 95-102. 
CHAPTER 7

Summary 
This thesis focuses on the fundamental understanding of phenomena associated with the gelation of end-functionalized polymers and the dynamic processes occurring inside of the gel network. To address particular questions we use two types of telechelic polymers, in which the assembly occurs due to the solvophobic interactions and due to the metal-ligand coordination, respectively. In this research we employ a number of methods, mostly rheology and light scattering.

In Chapter 2 we investigate the rich multimodal dynamics of mixtures of hydrophobically modified mono- and difunctionalized polyethylene glycol (PEG) with the same hydrophilic-lipophilic balance. For our research we employ dynamic light scattering and rheology. The investigated difunctionalized polymer assembles into physical gels, consisting of transiently cross-linked flowerlike micelles. The rheological properties of such gels depend on the lifetime of the network bridges and are determined by the exchange kinetics of the associating blocks. In this chapter we describe the role of the connectivity within the network and we do that by varying the number of bridges, while the other conditions are kept constant. We observe that with increased fraction of monofunctionalized PEG, fewer bridges can be formed and the modulus and viscoelastic relaxation time decrease strongly. Dynamic light scattering measurements reveal a relaxation spectrum consisting of three relaxation modes, referred to as fast, intermediate and slow modes. Even though transient networks have been studied with dynamic light scattering before, the interpretation of the particular relaxation modes remains a subject of debate. Our strategy of systematic termination of the number of bridges results in disentanglement of the effects of concentration and connectivity on the dynamics. We found that the fast mode is hardly affected by the number of bridges, and can be attributed to the cooperative diffusion of micelles at distances where bridges are irrelevant. On the other hand, the intermediate and slow modes depend strongly on the connectivity. We speculate that the intermediate mode can be related to the rearrangement of bridges, with a time scale that is proportional to the rheological relaxation time, while the slow 
diffusive mode is ascribed to the diffusion of micelles or clusters of micelles through the viscoelastic matrix.

In Chapter 3 we show and explain how the critical gel state in transient polymer networks is a result of arrested phase separation. To prove this, we use a unique telechelic system that shows either homogeneous networks with socalled Maxwellian behavior, or phase separated networks with critical gel-like behavior, depending only on the association strength of the sticky end groups. The polymers used in these experiments consist of flexible water-soluble polyethylene oxide (PEO) chains of $10 \mathrm{kDa}$, end-functionalized with metalbinding terpyridine (tpy) groups. The terpyridine end groups form tridentate coordination complexes with bivalent transition metal ions $\left(\mathrm{M}^{2+}\right)$. When dissolved in apolar media, such complexation would lead only to chain extension of the (tpy) $)_{2} \mathrm{PEO}$ telechelics due to the $1: 2 \mathrm{M}^{2+}$ :tpy binding stoichiometry. When water is used as a solvent, however, hydrophobic interactions between the conjugated coordination complexes provide an additional association mechanism, resulting in the formation of transient networks. Such association appears as a nanoscopic phase separation that enhances the mechanical properties of networks. Our findings suggest a dynamic, transient nature of the phase separation, where the equilibration depends strongly on the ratio of phase separation kinetics and the characteristic time scale of bond rupture enabling mechanical relaxation. Therefore, for systems that relax slowly but at finite time scales we find that the critical state is transient and vanishes as thermal rearrangements homogenize the phase separated structure towards its equilibrium, Maxwellian, state. Contrariwise, for systems where the binding strength is so large that spontaneous bond dissociation is frozen, the critical state may be long-lived.

Chapter 4 summarizes an extensive rheological study on a supramolecular network formed by terpyridine functionalized water-soluble telechelic polymers introduced in Chapter 3. We study the rheological responses in both linear and non-linear regimes as a function of the ratio between metal and polymer and the overall concentration. Samples at low concentrations are viscoelastic liquids, 
while with increased concentration we approach the gel transition. The critical gel state reported in chapter 3 appears when the ratio between metal and polymer is optimal for forming $\mathrm{Ni}(\text { tpy })_{2}$ complexes, which is $1: 1$. However, already at a slight overdose of metal, the power law scaling characteristic for critical gels disappears, and the response resembles a more traditional viscoelastic material with a characteristic relaxation time. In the non-linear regime and at high deformation rates the samples show strong shear thinning. This indicates a shear-banding instability associated with large stress fluctuations and metastable states and, finally, the so-called Weissenberg effect. We find that the onset of the instability is in agreement with a model for transient networks based on stress-activated crosslink dissociation.

In Chapter 5 we describe the orthogonal and hierarchical self-assembly of fully transparent and self-healing polymer composites based on non-covalent interactions exclusively. We employ a strategy based on the spontaneous generation of multivalent nanoparticle tectons, which we subsequently link together into network structures, using metal-coordination linkages proposed previously in Chapter 3 and 4 . The resulting materials are highly tunable, with moduli and viscosities ranging over many decades, without any additional chemistry, and display rapid, complete and reversible self-healing.

Finally, in Chapter 6 we discuss the results obtained in this thesis, by highlighting outstanding questions and by pointing out future directions for research on supramolecular gels. 


\section{About the author}

Małgorzata Anna Bohdan was born on February 9 $9^{\text {th }}, 1983$ in Kędzierzyn-Koźle, Poland. Until she was 19 she was living in Zdzieszowice, an industrial Silesian town in southwestern Poland. After finishing her secondary education in Liceum Ogólnokształcące im. Komisji Edukacji Narodowej in Gogolin, in 2002, she started her studies of Medical Physics at Jagiellonian University, Kraków, Poland. After 3 years she made a decision of changing the specialization into the Condensed Matter Physics. This is when she also grow her curiosity towards Soft Advanced Materials and polymers especially. In 2009 she started her MSc project under supervision of Prof. Monika Marzec and Prof. Stanisław Wróbel in the Departament of Advanced Materials Engineering, UJ, where she focused on dielectric and electro-optical studies of chiral thioesters. After graduation in 2010, she moved back to Zdzieszowice with an idea of continuing scientific career abroad, this time strictly in polymeric sciences. That happened in 2011 when she left to the Netherlands, where she started her doctoral studies in The Laboratory of Physical Chemistry and Colloid Science in Wageningen. Her work was conducted under the supervision of Prof. Jasper van der Gucht and Dr. Joris Sprakel for whom she was exploring the phenomenon of physical gelation of telechelic polymers. The most important results of this research are described in this thesis. In May 2016 she started her work as a scientist at DSM Coating Resins in Waalwijk. In her free time she's devoted to her passions in early music, visual arts and sports. 



\section{List of publications}

Chapter 2: M. Bohdan, J. Sprakel and J. van der Gucht. Multiple relaxation modes in associative polymer networks with varying connectivity. Submitted 2016

Chapter 3 and 4: M. Bohdan, J. van der Gucht, and J. Sprakel. Critical polymer networks by arrested phase separation. Submitted 2016

Chapter 5: M. Gerth, M. Bohdan, R. Fokkink, J. van der Gucht \& J. Sprakel. Supramolecular Assembly of Self-healing nanocomposite hydrogels. Macromolecular Rapid Communications, 35(2014), 2065-2070. DOI: 10.1002/marc.201400543.

Other work:

M. Marzec, M. Bohdan, M. D. Ossowska-Chruściel, J. Chruściel \& S. Wróbel. Dielectric and Electrooptic Studies of Chiral Thioester. Molecular Crystals and Liquid Crystals, 540(2011), 227-238. DOI:10.1080/15421406.2011.568889 



\section{Acknowledgements}

And now the entertainment part! I highlighted most of the names so you can skip the blabla and find yourself easier.

Almost five years ago I stepped out from the Ede-Wageningen bus with my $40 \mathrm{~kg}$ suitcase that eventually crashed my feet. I also remember the rain and lack of umbrella, and of course unique beauty of my new town. That would be lie if I would say I wasn't shocked! After 8 years of living as a city rat I ended up, well, here... After 4.5 years, however, I learned to love this place. It's even hard to call it a place when Wageningen is nearly a statement! In the end, to Wageningen I owe the most amazing and inspiring time of my life. I wrote my $\mathrm{PhD}$ thesis here, defined myself as a scientist, met friends for life and, what's essential, my future husband.

But it wouldn't happened without a real army of unique people located in and out of this genuine Dutch gem.

First of all I, wanted to thank to my first supervisor Prof. Monika Marzec from my Alma Matter, Jagiellonian University in Kraków, Poland. Dear Mam, you were the one that trusted in me from the very beginning of my scientific career. My time spent at ZINM UJ gave me something that I never had before, a confidence and eagerness for reaching higher and higher. Thank you for that!

Second thanks, equally important goes to my Wageningen bosses. Having two supervisors might be in many cases a challenge, yet I feel blessed that I had a chance to cooperate with two such different and unique scientists. You both present so diverse management styles and research philosophies, that when put together it creates an ultimate, multilayered, highly explosive scientific combo. Jasper, I admire your analytical mind and strong theoretical background. You always could embrace all the data I produced with such an easiness. Thank you for all our discussions and your patience with correcting my poor English. Joris, I was always amazed with your enthusiasm and power of expression. Your intriguing personality and empathy motivated me from the day I started my 
PhD. I am really grateful that you joined the project. We wouldn't make it without you putting your hart and your energy in it.

Josie, woman with a property of a Band-Aid, thank you for your warmth and kindness. You've been always there ready to help, listen and cheer up when crying. During this four years you shaped my expectations towards secretaries, Josie's style should be a standard in all the institutions! Mara, you showed me there are no excuses for being out of style. Even the hardest job can be done in high-heels and pearl-neckless. But apart from your style I want to thank you for your beautiful personality. Also when I look at you and your husband together I want to say "wooooow, aren't they amazing?" Thank you for being my role model on all the layers of womanhood. You are a real icon! Remko, I don't know what I would do without your assistance. You always had an answer for any question that appeared during all those DLS measurements. I hope everyone knows that you are a genuine superhero. Hans, you are the essence of the Fysko team. It is a pleasure an honor to know you. Thank you for your precious suggestions during colloquia and you endless happiness. Herman, thank you for your sense of irony. You know, sarcasm feels like a little verbal hug. Frans, thank you for thinking always outside of the box. Having you as a colleague is a precious lesson in human and in scientific sense.

This booklet wouldn't happened without support and help of many of my colleagues and friends. People, I love you all, time spent in Wageningen wouldn't be that same without you. First of all of them, Katarzyna, jakbym miała siostrę to chciałabym by była taka jak Ty. Dziękuję za wszystkie nasze rozmowy i dzikie eskapady, za Wadlopen, Alpy, narty w środku lata, porwanie dziwnego pana z konferencji, prezydenta Cynamona, szaloną noc przy tequili i wiele innych. Szczególnie dziękuję Ci za Twoją intuicję co do Kamurana i przyczynienie się do największego szczęścia jakie mnie właśnie spotyka. Zawodowo dziękuję Ci, że byłaś mi drogowskazem zaraz na początku mojej chemicznej przygody. Dzięki za Twoje trwarde osądy i wielkie doświadczenie, uczyniłaś mój start w Wageningen dużo łatwiejszym. Dmitry, you are very special person to me. Thank you for all the talks about faith and philosophy, for your open mind and selflessness. You are an amazing scientist and human being. Rui, you weirdo! In the end I believe we are both very similar, we just manifest it differently. I'm very happy that I know you, and not only cause of 
your funny name. Thank you for helping me with designing the cover. Frank, grumpy cat seems amateur when placed next to you. I enjoyed us working together very shortly, I hope we will meet again one day. Hande and Eric, thank you for becoming my friends in the last stretch of my Fysko path, for your witty minds reminding me that it's not an ordinary job, but still an academia. For our talks and our tears, and of course, I wouldn't miss that, for bringing Pamuk to our (I mean globally) lives. She is a very special lady, everyone will admit that! Inge, so pity that we came closer just in the end of our roads. You are such a wonderful person, thank you for your hardworking and sportive attitude!

I want to say thanks also to the other members of Fysko family. Starting from all my office-mates: Monika, you welcomed me just at the beginning of my $\mathrm{PhD}$, thank you once more for your and Diegos hospitality, Juan, thank you for all the discussions on the Asian/European borderline, Natalia, for creating focused and hardworking environment in 1008 and of course for your priceless craziness!! And of course Hande, once more (-). Ula, that was short time together but I felt we clicked in very special way, I wish you were here. Soumi \& Surender, you both are like from the other world, you were Vulcans of Fysko, thank you for the real intellectual attitude, questioning the unquestionable, and of course for the most amazing laughter I ever heard. Rechdev (-) thanks for being the only antibolshevic at this side of the Oder river, I cri evertim. Thao, I miss you so much! There's not so many personalities that combines sweetness and wisdom, thank you for that. Merve, for being another manifestation of turkishness in my life. Sabine, my inspiration to become vegetarian, thanks also for the US PhD trip, would it be possible without you? And by the way, I just love to see you as a mother. Liyakat, for your kindness and crossing the ideological borders, Jacob, for your contentious laughter, Huanhuan \& Tingting, for your sweetness and kind words each time I met you, Wolf, for the spirit of competition, Yunus, for assembling the wardrobe with me and all the talks about arts and music. Armando, for unlimited energy, Gosia, thanks for the Mexico trip, Lennart, you loved that one, didn't you? But seriously, thanks for having heart on the correct side of the body. Harke, for our endless talks, Johan, we've been always shutting down the lights as the last ones in our corridor, Marcel, you are such an 
awesome guy, pity we didn't have more time to get to meet each other. Joshua, thanks for the crazy Nantes trip, you remind me my friends I left in Kraków, Marleen, thank you for cheering me up at the very important moment of my career.

Also thanks to: Anita, Anton, Antje, Bert, Celine, Christian, Diane, Dirk, Hanne, Hannie, Ilse, Evan, Jan, Jan Bart, Jan Maarten, Jeroen, Junyou, Maria, Maarten, Martin, Nadia, Lione, Peter, Rene, Ronald, Ruben, Ties, Vittorio.

Many thanks goes also to my new colleagues from DSM. First of all, thank you for trust in me, second, for such a wave of kindness and warm welcome. I didn't expect that!! I enjoy my time with you so much, I hope our cooperation will grow and very soon will bring an amazing effects in a field of colloidal sciences.

Many thanks also to my friends in Poland. To, że przy mnie trwacie to jedno z największych błogosławieństw jakie mogło mnie spotkać. Wszystkich Was w tym paragrafie kocham z całego serca! Mateuszu, dzięki za te wszystkie godziny spędzone na skype i Twoją gościnność w Warszawie. Musiałeś się sporo nasłuchać podczas wszystkich moich wzlotów i upadków, dlatego dziękuję też za cierpliwość. Jestem niezwykle dumna, że mam Cię za przyjaciela! Kasiu i

Piotrze, Maju i Piotrze dziękuję, że jesteście, mimo że nie zawsze jest czas żeby się zobaczyć czy nawet zdzwonić. Jesteście dla mnie jak rodzina z wyboru. Dzięki za weekendy spędzone w Paryżu i w Krakowie. Kasiu, szczególnie Tobie dziękuję Twoje wielkie serce i Twoją empatię! Magda i Adrian, dzięki za wielki come-back w moim życiu. Dzięki wszystkim których spotkałam na swojej drodze, którzy w większy lub mniejszy sposób wpłynęli na moją osobowość, wzmocnili wiarą lub też niewiarą.

Special thanks to my almost family in law (Kamu, please translate (:)). Especially to my future parents in law for hospitality, kindness, acceptance and all the priceless help on every step we were taking. Thank you for making my home here in Netherlands. That same appreciation goes to Yasin and Meral for being there always when we were hopeless. Nuran, Kudret, Nurten, Pablo, Atalay, thanks for being my cousins in law, I'm so happy to have you in my life. 
Na koniec kącik vipowski. Mamo, tato, dziękuję za Wasze wsparcie i pogodę ducha w nawet najcięższyc sytuacjach. Szczególnie dziękuję za ducha wolności jaki mi wpoiliście, dzięki niemu mogłam zrobić coś tak zwariowanego jak kariera i życie za granicą. To po Was odziedziczyłam ciekawość świata, więc jakby co, to wszystko Wasza wina.

Łukasz i Justyna, dzięki za całą pomoc jaką od Was otrzymałam jeszcze w Krakowie. Bez Was nie zrobiłabym następnego kroku jakim był doktorat. Dzięki, za nauczenie mnie jaką wartośca jest precyzja i tego, żeby zawsze wymagać. No i dzięki za Franka i Wojtka, za uświadomienie mi, że można kochać za samo istnienie.

Wszystkim dziadkom za wpojenie ducha pracy, bezwarunkową miłość i nieograniczoną wiarę.

Finally, I want to thank to Kamuran. Because of you I know what the difference is between lepidocrocite and goethite, cirrostratus fibratus and cirrocumulus floccus. I know the speciation of all the transition metal ions, well, I know the "speciation" word actually exist!! I can explain occluded front and tell why there is so many fish in the coast of Senegal. Also you taught me I should always ask about the $\mathrm{pH} /$ charge ratio/ionic strength, because "no one considers that anymore!!!"

But let's be serious in this last paragraph. You were always there, as the only one knowing how much this thesis cost me. Thank you! It is cause of you I can handle it today. You believed in me when everyone else (including me) lost the hope. Way back, four years ago I couldn't imagine I will be so blessed to share my life with you, having your ultimate friendship and love. The new, great era started when you happened in my life. Let's keep it this way. 
Overview of completed training activities

\section{Discipline Specific}

Winterschool of Physical Chemistry

Han-sur-Lesse (Belgium) 2012

PTN Polymer Physics

Utrecht (The Netherlands) 2012

Polymeric and self-assembled hydrogels*

London (United Kingdom) 2012

Physics @FOM*

Veldhoven (The Netherlands) 2013

International School of Rheology

Leuven (Belgium) 2013

International Soft Matter Conference*

Rome (Italy) 2013

Physics of Soft and Biological Matter*

Cambridge (United Kingdom) 2014

Dutch Polymer Days $\dagger$

Lunteren (The Netherlands) 2015

The Annual European Rheology Conference $\dagger$

Nantes (France)

\section{General}

Journal Club

Wageningen

2012-2015

Presentation skills

Wageningen

2012

Interpersonal Communication

Wageningen

2013

for PhD students

Career Perspectives

Wageningen

2014

Mobilizing your scientific network

Wageningen

Project and time management

Wageningen

\section{Optionals}

Preparation for research proposal

Weekly work meetings and colloquia

Wageningen

PhD study trip

USA

* poster presentation $\quad \dagger$ oral presentation 
Cover design:

Printed \& Lay Out by:

Published by:
Małgorzata A. Bohdan \& Rui Carvalho

Proefschriftmaken.nl

Proefschriftmaken.nl 